INTER NATIONAL MONETARY FUND



Staff






\title{
Islamic Republic of Afghanistan: First Review Under the Extended Credit Facility Arrangement, Request for Waiver of Nonobservance of a Performance Criterion, Modification of Performance Criteria, and Rephasing of Disbursements-Staff Report; Staff Supplement; Staff Statement; Press Release on the Executive Board Discussion; and Statement by the Executive Director for the Islamic Republic of Afghanistan.
}

In the context of the Islamic Republic of Afghanistan: First Review Under the Extended Credit Facility Arrangement, Request for Waiver of Nonobservance of a Performance Criterion, Modification of Performance Criteria, and Rephasing of Disbursements, the following documents have been released and are included in this package:

- $\quad$ The staff report for the Islamic Republic of Afghanistan: First Review Under the Extended Credit Facility Arrangement, Request for Waiver of Nonobservance of a Performance Criterion, Modification of Performance Criteria, and Rephasing of Disbursements, prepared by a staff team of the IMF, following discussions that ended on May 16, 2012, with the officials of Afghanistan on economic developments and policies. Based on information available at the time of these discussions, the staff report was completed on June 19, 2012. The views expressed in the staff report are those of the staff team and do not necessarily reflect the views of the Executive Board of the IMF.

- $\quad$ A staff supplement dated June 19, 2012 containing a joint World Bank/IMF Debt Sustainability Analysis.

- $\quad$ A staff statement of June 28, 2012 updating information on recent developments.

- $\quad$ A Press Release summarizing the views of the Executive Board as expressed during its June 29, 2012 discussion of the staff report that completed the request and/or review.

- A statement by the Executive Director for the Islamic Republic of Afghanistan.

The documents listed below have been or will be separately released.

Letter of Intent sent to the IMF by the authorities of Afghanistan*

Memorandum of Economic and Financial Policies by the authorities of Afghanistan* Technical Memorandum of Understanding*

*Also included in Staff Report

The policy of publication of staff reports and other documents allows for the deletion of market-sensitive information.

\author{
Copies of this report are available to the public from \\ International Monetary Fund $\bullet$ Publication Services \\ $70019^{\text {th }}$ Street, N.W. $\bullet$ Washington, D.C. 20431 \\ Telephone: (202) 623-7430 • Telefax: (202) 623-7201 \\ E-mail: publications@imf.org Internet: http://www.imf.org
}

\section{International Monetary Fund Washington, D.C.}


INTERNATIONAL MONETARY FUND

ISLAMIC REPUBLIC OF AFGHANISTAN

\title{
First Review Under the Extended Credit Facility Arrangement, Request for Waiver of Nonobservance of a Performance Criterion, Modification of Performance Criteria, and Rephasing of Disbursements
}

\author{
Prepared by the Middle East and Central Asia Department \\ (In consultation with other Departments)
}

Approved by Adnan Mazarei and Dhaneshwar Ghura

June 19, 2012

\begin{abstract}
Discussions: In May 2012, a mission met in Kabul with Minister of Finance Zakhilwal, Governor Delawari, Senior Minister Arsala, the Attorney General, and other high-ranking government officials, as well as donors, and private sector representatives.

Staff Team: The mission consisted of Mr. Axel Schimmelpfennig (Head), Ms. Melissa Tullis (LEG), Messrs. Aidar Abdychev and Marcin Sasin (all MCD), Ms. Jeta Menkulasi, Mr. Aqib Aslam (all FAD), and Mr. Warren Coats (MCM consultant). The mission was supported by Mr. Wabel Abdallah, Resident Representative, Mr. Nabil Hashad (MCD long-term advisor), and Ms. Mary Woolford (MCM consultant) in Kabul, and Ms. Katrin Elborgh-Woytek (SPR) in Washington, D.C.
\end{abstract}

Exchange Arrangement: Afghanistan maintains a floating exchange rate regime, and is an Article XIV member country. The authorities believe that they are implementing an exchange system without exchange restrictions and have requested a Fund review of laws and regulations on the exchange regime for accession to Article VIII status. Staff is currently conducting this review in order to assess the jurisdictional implications of the exchange system of Afghanistan.

Past Surveillance: The 2011 Article IV Consultation was concluded on November 14, 2011, and the report is available at http://www.imf.org/external/pubs/ft/scr/2011/cr11330.pdf

Statistics: Data provision has significant shortcomings, but is broadly adequate for surveillance and program monitoring. The authorities are receiving IMF assistance on national accounts, consumer prices, monetary statistics and the balance of payments and Government Financial Statistics, as well as donor support in other areas of statistics. Afghanistan is a participant in the General Data Dissemination Standard (GDDS). 


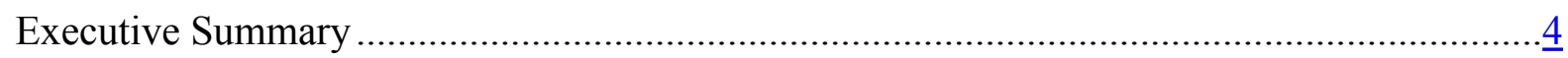

I. Background: Afghanistan Sets Out on a Path Toward Self-Reliance …............................... $\underline{5}$

II. Recent Economic Developments and Outlook...................................................................

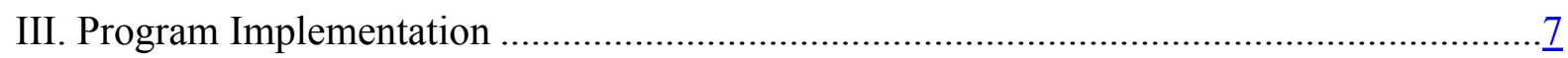

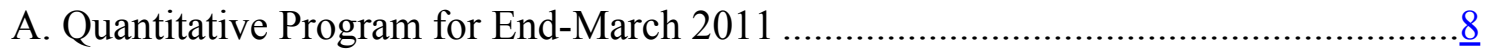

B. End-December 2011 and End-March 2012 Structural Benchmarks ............................ 9



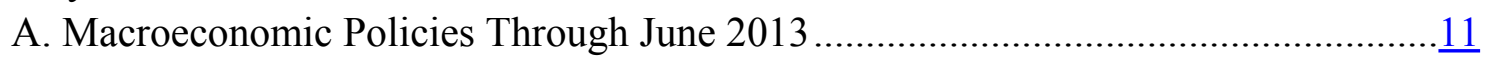

B. Safeguarding the Financial Sector................................................................... $\frac{12}{13}$

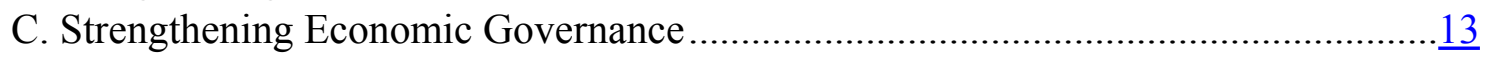

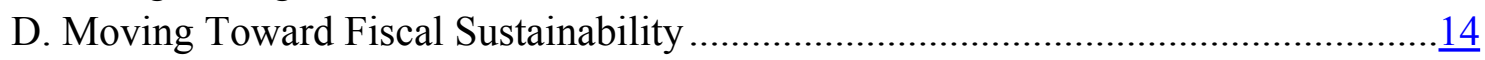

E. Fiscal and External Debt Sustainability Analysis .................................................14





Figures

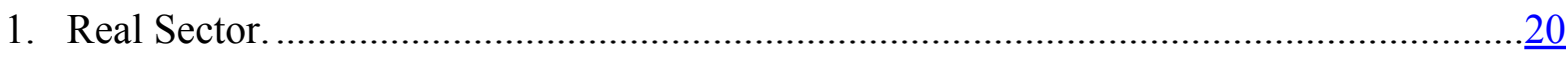

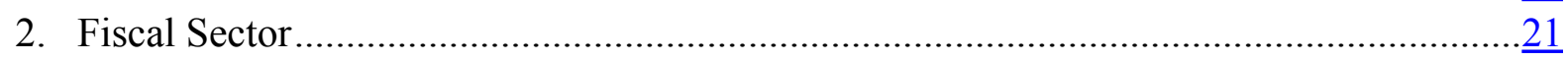

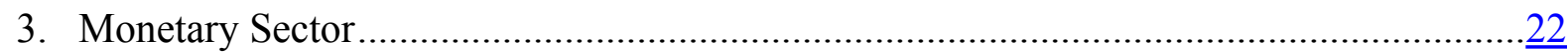

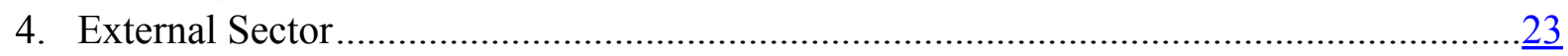

Tables

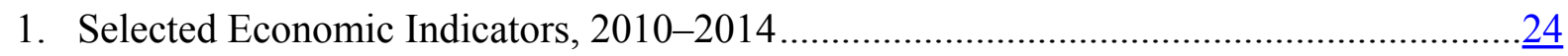

2. Medium- and Long-Term Macroeconomic Framework, 2011-2025 …..........................25

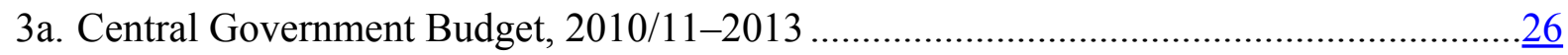



4. Total On-Budget and Off-Budget Fiscal Financing Needs, 2012-25 ...............................



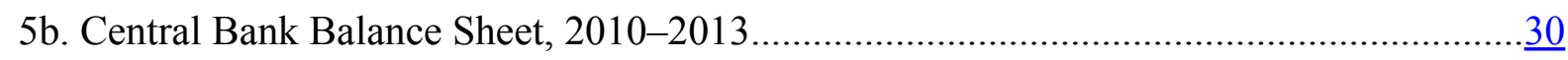

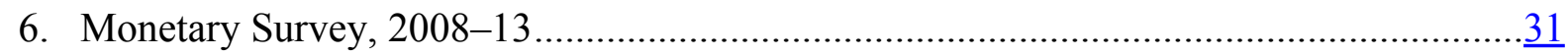



8. Proposed Schedule of Reviews and Disbursements Under the ECF Arrangement ..............33

9. External Financing Requirement and Sources, 2010-14 ..............................................34 
Appendix

I. Letter of Intent

Appendix Tables

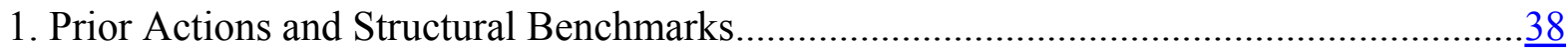

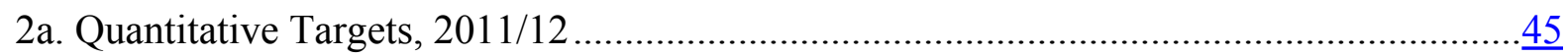

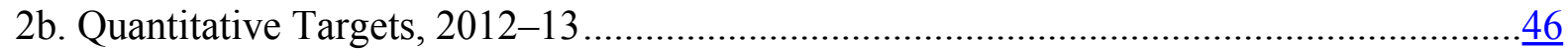

3. Rephasing and Change to the Schedule of Reviews .......................................................4

Attachments

I. Memorandum of Economic and Financial Policies................................................ 48

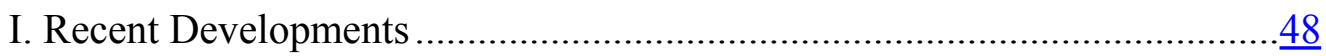

II. Program Implementation Through June 2012 …….................................49

A. Quantitative Program.................................................................. 49

B. Structural Reforms ..............................................................

III. Economic Program Through June 2013 ..................................................

A. Macroeconomic Policies ………………………......................52

B. Structural Reforms …….............................................................

IV. Program Modalities and Monitoring …………....................................

II. Technical Memorandum of Understanding ……...................................................61

I. Program Exchange Rates and Gold Valuation .........................................61

II. Quantitative Performance Criteria and Indicative Targets..........................61

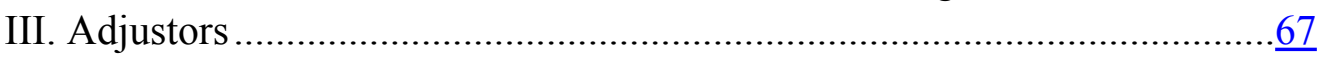

IV. Provision of Information to the Fund ..................................................68 


\section{EXECUTIVE SUMMARY}

Afghanistan is embarking on a very difficult path toward self-reliance. The country faces widespread underemployment and poverty, and a challenging security situation and economic governance issues. Sound macroeconomic policies are important for economic development and poverty reduction, but noneconomic factors can frustrate policy efforts and even reverse hard-earned gains. Donor engagement will be a necessary condition for success.

To be successful, the country will need high and prolonged donor support. The security handover from the International Security Assistance Force (ISAF) is about 75 percent complete, and expected to be finalized by end-2014. Insurgent groups remain very active, and continue to stage high profile attacks including in Kabul. The May 2012 NATO summit in Chicago generated commitments for security aid - about US\$3 billion annually through 2017 (or 15 percent of GDP in 2012) — but donors called for Afghanistan to become selfreliant over the coming decade. At the July Tokyo Conference, Afghanistan will seek donor support for its development agenda of about US\$4.4 billion annually through 2015.

\section{In this context, the IMF-supported economic program tries to address key}

macroeconomic challenges. The program provides the framework to manage the economic impact of the withdrawal of foreign troops, and identifies financing needs and sources.

Moreover, the program seeks to safeguard the financial sector, strengthen economic governance, and move toward fiscal sustainability.

However, program implementation to date has been weak and raises concerns. The authorities have completed all but one of the structural benchmarks for the first review, but with delays. Cash recoveries from Kabul Bank reached US\$128 million through June 4, 2012 out of a total of US\$935 million owed to the receiver; the authorities will seek further recoveries through legal channels. They also request additional time for two benchmarks set for the second review. The limited technical capacity and the heavy workload in the run-up to the Tokyo Conference partly explain the slow reform pace. The lack of ownership by the government at large and opposition from vested interests are also formidable obstacles.

\section{Looking ahead, Afghanistan faces pressing challenges that require determined policy} implementation. Corruption and poor governance take their toll on the economy. Moreover, weaknesses in the banking sector are a major risk that needs to be addressed urgently. Over the medium term, fiscal pressures will emerge, leading to a fiscal financing gap of over 20 percent of GDP over the coming decade and beyond.

Notwithstanding the delays in program implementation, staff recommends the completion of the first review and supports the authorities' request for a waiver. The authorities made substantial progress on their reform objectives under the program during difficult times. Going forward, the program provides the macroeconomic anchor in challenging waters, addresses the key issues facing Afghanistan, and helps generate the necessary political support for this reform agenda. Risks to the program are very high, and future slippages cannot be ruled out. Overall program implementation needs to improve considerably before the second review. 


\section{Background: Afghanistan Sets Out On a Path Toward Self-Reliance}

1. The security and political situation remains very difficult. Insurgents continue to stage high-profile attacks, including in Kabul. Recent targets include foreign embassies and a high-ranking member of the High Peace Commission which is supposed to engage in talks with the Taliban. The 2014 presidential election may impact the pace of reforms.

\section{Afghanistan development, humanitarian and governance challenges are} formidable. After decades of war, Afghanistan ranks in the bottom 5 percent of countries on the 2011 UN Human Development Index. Unemployment and underemployment are widespread, the poverty rate was 36 percent in 2007/08, and female literacy (ages 15-24) stood at only 22 percent. Key problems include large and active militant groups, poor governance, low levels of human capacity, a massive illicit sector, a large cash based economy, and porous borders. This, combined with the post-2001 surge of military, development, and humanitarian aid has contributed to distorted economic incentives, rentseeking behavior, and helped entrench powerful vested interests.

\section{Afghanistan is at an important juncture in its transition to self-sustained} security. The hand-over of security responsibilities from ISAF to the government is well advanced. Afghan forces are now in charge of three quarters of the country, and ISAF intends to withdraw most foreign troops, end combat operations, and limit their activities to training by December 31, 2014. By 2017, the costs of the Afghan police and military are projected to be US $\$ 4.1$ billion. At the May 2012 NATO summit in Chicago, donors pledged to contributed about US $\$ 3$ billion, and asked that Afghanistan gradually phase out its need for security aid by 2025 . Unless the security situation were to stabilize much faster than anticipated and security costs turn out lower than the projected $14 \frac{1}{2}$ percent of GDP, this could be very difficult, given that domestic revenue (including from natural resources) is likely to reach only 17 percent of GDP by that time.

\section{Next to security, development is the second overarching policy objective for} which the government will need donor support. The authorities have prioritized the Afghanistan National Development Strategy (ANDS), embedding it into 22 National Priority Programs (NPPs). Even with this prioritization and sequencing, the authorities ambitiously target development spending at US $\$ 4.4$ billion annually in the coming years. They seek donor support for this development agenda beyond 2014 at the forthcoming Tokyo Conference on July 8, 2012. Donors have stated that they are looking for tangible progress on governance before making long-term commitments. Ultimately, development and the security situation will evolve interdependently either in a virtuous or vicious circle. Hence, finding the appropriate balance between these two key objectives will be important. 
5. Against this background, the IMF's Executive Board approved a three-year Extended Credit Facility (ECF) arrangement for Afghanistan on November 14, 2011. ${ }^{1}$ The main goals of the program are to make progress toward a stable and sustainable macroeconomic position, move toward fiscal sustainability, strengthen the financial sector, and improve the transparency and effectiveness of public spending while protecting the poor, and strengthening the governance framework in the financial and economic sphere. The resolution of failed Kabul Bank, including asset recovery, features prominently throughout the program to ensure that accountability and the rule of law are enforced, and that fiscal costs are contained.

\section{RECENT ECONOMIC DEVELOPMENTS AND OUTLOOK}

\section{Macroeconomic developments in 2011 were mixed, but the economy was} sustained by donor inflows. Real GDP growth slowed to around 6 percent, from over 8 percent in 2010, reflecting a poor harvest. After dropping into single digits during 2011, inflation accelerated again in recent months, reaching 11 percent year on year in March. Nonfood inflation-14 percent in March — remains persistently high. Exports weakened, but the current account deficit of about 40 percent of GDP (excluding grants) was more than financed by aid inflows. Gross international reserves increased to US $\$ 6.2$ billion (7 months of imports). The exchange rate has depreciated by 10 percent during 2011 -driven by higher than programmed purchases by the central bank-but has remained stable, at around 50 Afghanis per U.S. dollar, for the past six months. Deposits in the banking sector have stagnated at about 20 percent of GDP since mid-2011, reflecting a continued lack of confidence. There has also been virtually no new net bank lending during the past year, mostly for lack of sound lending opportunities and the general economic and political uncertainty. The interest rate on the central bank's 28 -day notes has remained stable, at around 2 percent.

\section{In 2012, agriculture should more than offset the drag on growth from the} external economic environment. The global and regional economic environment is difficult, with elevated oil prices and occasional trade disruptions with neighboring countries. Domestically, the low level of security and the impact of the ongoing military withdrawal also hold back activity. However, agriculture is expected to rebound and boost real GDP growth to about 7 percent. With international commodity prices set to stabilize, inflation could decelerate to $7 \frac{1}{2}$ percent year-on-year by end-2012. Exports are likely to be sluggish due to weak external demand, while the higher fuel prices will inflate Afghanistan's import bill. The resulting large trade deficit will continue to be covered with grants.

\footnotetext{
${ }^{1}$ See IMF Country Report No. 11/330 (http://www.imf.org/external/pubs/ft/scr/2011/cr11330.pdf).
} 


\section{Program Implementation}

\section{The authorities have broadly met their program objectives, but implementation} has suffered from delays. The authorities have achieved their end-March quantitative targets in the monetary sector (Table 2 of the attached letter of intent). However, the revenue target has been missed by a small margin. Progress has also been made on a number of structural reforms, including on introducing a value-added tax (VAT) and establishing an agreed-upon capitalization framework for the central bank (Table 1 of the attached letter of intent). Notwithstanding this, a number of structural benchmarks have been implemented with long delays or need follow-up actions to make them consistent with program objectives; one structural benchmark will only be fully completed later this year.

\section{Importantly, the authorities have now initiated an asset recovery process for} each Kabul Bank beneficiary. Through a Presidential Decree, the authorities modified their asset recovery strategy on April 4, 2012. Previously, they relied on legally binding agreements with former Kabul Bank shareholders and related parties for repayment of the amounts owed. ${ }^{2}$ However, actual repayments under these agreements fell significantly short of obligations, and the authorities concluded that the approach was not working. Instead, President Karzai gave Kabul Bank shareholders and related parties until June 4, 2012 to repay in full the amounts attributed to them in a forensic audit report. Failing to do so would result in the beneficiary being referred to a special tribunal as a civil or criminal case. As the authorities report in the attached letter of intent (LOI) and paragraph 14 of the attached memorandum of economic and financial policies (MEFP), cash recoveries on June 4, 2012 had reached US\$128 million out of US\$935 million total receivables identified in the forensic audit report - about US\$54 million since the program approval in November 2011, of which about US\$30 million were collected since the presidential decree, including US\$5 million from regular borrowers. No beneficiary identified in the forensic audit report has paid in full. The two main architects of the fraud have been referred to the special tribunal, and the remaining beneficiaries will be dealt with by the Financial Dispute Resolution Committee, and, if warranted, could be referred to the special tribunal at a later date. The Financial Dispute Resolution Committee has been working closely with the receiver on asset recovery, and is the appropriate institution to rule on civil cases. The authorities have also issued requests for mutual legal assistance requests to the United Arab Emirates to recover assets outside of Afghanistan. Lastly, they will report public on asset recovery going forward.

10. Staff welcomes the progress made, but has concerns over the quality of the steps taken. In particular, staff notes that the referrals to the special tribunal make no mention of the evidence of the forensic audit report. This is surprising, given that, at the time of the

\footnotetext{
${ }^{2}$ A prior action on asset recovery for the approval of the ECF arrangement was only partially completed at the time. In approving the program, Executive Directors requested that the measures be completed before the first review.
} 
program request, the authorities argued that they needed more time to show progress on asset recovery until the results of the forensic audit would be available. Staff also notes the limited legal capacity to pursue asset recovery, and the lack of clarity over the role of the Financial Dispute Resolution Committee. Moreover, it is unclear whether the government at large has the necessary political will to pursue asset recovery through the legal system in earnest. As such, there is a risk that the process initiated now will not lead to substantial asset recovery beyond what has been collected to date.

\section{A. Quantitative Program for End-March 2011}

\section{Monetary policy was tightened appropriately given the renewed acceleration of} inflation. The central bank reduced reserve money growth to 16 percent year-on-year in March 2012 (compared to over 21 percent for the previous year and the 19 percent ceiling envisaged under the program). Currency in circulation grew by 12 percent year-on-year, below the indicative ceiling. On the back of strong financial inflows, net international reserves reached US\$5.9 billion at end-March 2012, or US\$220 million over the program floor.

12. Fiscal developments were marked by a shortfall in revenue collection. While revenue collection edged up marginally to 11.1 percent of GDP in 2011/12 from 11.0 percent of GDP in 2010/11, the program target was missed by a small fraction because of unanticipated disruptions at the border with Pakistan and problems in revenue administration. Given their objective to gradually increase the revenue to GDP ratio, the authorities have committed to phasing out the use of "concessions" which allow certain taxpayers to pay a minimum presumptive tax rather than file for corporate income tax and business receipts tax. The revenue shortfall also meant that the operating budget deficit at 6.1 percent of GDP slightly exceeded the indicative program target, despite expenditure restraint in the first half of the fiscal year. Development spending increased by 22 percent in 2011/12, reaching 6 percent of GDP. Encouragingly, better capacity in line ministries and more realistic budgeting led to an implementation rate of 52 percent. Social spending reached 2 percent of GDP meeting the program target. As the budget had sufficient external financing, net central bank credit to the government remained within program targets.

\section{The authorities refrained from nonconcessional borrowing and did not}

accumulate arrears. Consistent with the program, no new nonconcessional external debt, no short-term external debt and no new external arrears have been incurred. Afghanistan remained in compliance with its Article VIII commitments. ${ }^{3}$

\footnotetext{
${ }^{3}$ Since April 2012, a cabinet decision restricts the amount of cash a person can take out of the country to US $\$ 20,000$ per trip. The decision aims to tackle illicit money transfers. It has not yet been reflected in the laws. Given that there are no limits on the making of payment or transfers out of the country through the banking system, including through credit cards, this measure does not give rise to an exchange restriction on the making
}

(continued) 


\section{B. End-December 2011 and End-March 2012 Structural Benchmarks}

\section{The ministry of finance and the central bank signed a memorandum of} understanding on central bank capitalization framework in May. This memorandum, inter alia, aligns the framework with good international practices and recommits parties to fully implement the recapitalization provisions as soon as the required amendments to the central bank law have been approved. In parallel, on May 21, 2012, Da Afghanistan Bank (DAB) retained KPMG as its external auditor for its 2011/12 financial statements. The auditor had already been present at the end-year cash count. The appointment had been delayed since December 2011 by the council of ministers which has to approve DAB's proposal.

15. The draft VAT law was submitted to the ministry of justice for legislative review in December 2011. ${ }^{4}$ Although broadly consistent with Fund advice, the law contains some clauses, such as giving the minister of finance authority to grant VAT exemptions, which risk undermining the effectiveness of the VAT and need to be revisited. The authorities have committed to addressing the issue by limiting exemptions in the law and requiring that additional exemptions can only be introduced with parliamentary approval and have to be included in the VAT (structural benchmark for the third review).

16. New Kabul Bank has been operating broadly according to its business plan. By February, 25 branches of New Kabul Bank have been closed, deposit rates have been reduced, deposits have stabilized at around US\$600 million (somewhat higher than previously expected) and expenses have been cut substantially. In anticipation of privatization, the bank is not engaging in any new lending and, therefore, making monthly losses of around US\$2-3 million.

17. A national strategy to build capacity and improve the institutional framework to prevent and effectively respond to economic crimes was finalized in May 2012. While the strategy took longer than planned, it addresses gaps in existing governance programs and prioritizes addressing economic-governance related vulnerabilities in institutional frameworks. The strategy was included in the Governance National Priority Program "Justice for All."

18. Insufficient progress has been made on bringing state-owned enterprises and state-owned corporations under effective control of the ministry of finance. The authorities shared draft amendments to the law on state-owned enterprises with staff, and it was agreed that they fell short of the objective of bringing these enterprises under effective

of payments and transfers for current international transactions and is thus consistent with Afghanistan's Article VIII obligations.

${ }^{4}$ However, due to the unfamiliarity of the ministry of justice with the subject, the draft has been returned to the ministry of finance for further clarification. 
control of the ministry of finance. Staff has provided specific comments to strengthen the draft amendments. More importantly, amendments to the company law to bring state-owned corporations under the effective control of the ministry of finance will take more time. The authorities have added a structural benchmark on this for end-December 2012. State-owned corporations pose a much larger fiscal risks and, therefore, need to be addressed urgently.

\section{The authorities also made welcome progress in a number of other structural} reform areas. The new border management model was rolled out to two additional bordercrossing points in March (structural benchmark). In the fiscal area, progress has been made in program budgeting, financial planning, medium-term fiscal framework, internal audit, and the reorganization of the customs department. In the banking sector, an audit of a bank found no fraud similar to that perpetrated at Kabul Bank - the bank has violated exposure limits, including to a related party, and some years back, the bank appears to have been involved in a misuse of loans, though these loans have been paid back. The bank is working closely with the authorities to address the various shortcomings and to return the bank to regulatory compliance, including through additional recapitalization, though the supervisor has given an extra three months (until June) for the capital injection agreed last year.

\section{Policy Discussions}

\section{Discussions centered on four areas:}

- Macroeconomic policy mix: The challenge is to assure continued donor support and balance security, social, and development spending needs within a limited domestic resource envelope, while building buffers and returning inflation to single digits. This would form the prerequisite for sustained inclusive growth and poverty reduction.

- Safeguarding the financial sector: The authorities will address structurally weak banks, strengthen the legal and regulatory environment of the financial sector, further enhance banking supervision, and sell or liquidate New Kabul Bank;

- $\quad$ Strengthening economic governance: Further progress on asset recovery from Kabul Bank through the special tribunal is the primary focus of the authorities. In addition, they will address deficiencies in economic and financial sector governance, including in their antimoney laundering and combating the financing of terrorism (AML/CFT) framework;

- Moving toward fiscal sustainability: The authorities will continue the work to introduce a VAT in 2014, develop a fiscal regime for natural resources, introduce sukuks, strengthen public financial management, and start tackling the fiscal risks from state-owned enterprises and corporations. 


\section{A. Macroeconomic Policies Through June $2013^{5}$}

\section{The authorities and staff agree that the macroeconomic policy mix remains} broadly appropriate. Fiscal policy focuses on development spending and revenue mobilization, while monetary policy focuses on inflation and financial stability. The operating balance excluding grants remains the fiscal anchor, and reserve money the monetary anchor. The authorities remain committed to a floating exchange rate regime that, combined with their international reserves buffer, will help cushion potential external and domestic shocks. They emphasized that, given the country's large security and development needs and limited domestic resources, they will need significant long-term donor support to meet their external and fiscal financing needs.

\section{Fiscal policy aims to increase the revenue-to-GDP ratio and further improve} development budget implementation capacity. The 2012 budget is consistent with the program (as initially designed for 2012/13, MEFP paragraph 18). Domestic revenue collection is expected to increase to 11 percent of GDP in April-December 2012-a transitional fiscal year-from 10.6 percent of GDP during the same period in $2011 .{ }^{6}$ Compared to a normal fiscal year, 2012 will see a temporary dip in revenue collection because it does not include January-March which is traditionally the strongest quarter. Development spending, including on health and education, is targeted to grow by about $1 / 2$ percent of GDP to 6.4 percent of GDP. The budget also includes an allocation of US\$65 million for the amortization of the promissory note issued to DAB for the lender of last resort loan to Kabul Bank. The authorities target an operating deficit (excluding grants) of 5.9 percent of GDP and an overall deficit (excluding grants) of 12.3 percent of GDP. The deficit will be covered by grants, and a small amount of concessional external borrowing.

23. Monetary policy will aim at reducing inflation (MEFP paragraph 19). Given the estimate for money demand and a need to accommodate a change in seasonality related to a shift in the fiscal year, the authorities and staff agreed that a reserve money growth of about 19 percent over 2012 should be sufficient to bring headline inflation down to about $71 / 2$ percent by end-2012. Foreign exchange auctions will continue to be the main policy instrument, complemented as needed with sales of capital notes.

\footnotetext{
${ }^{5}$ Afghanistan will change its fiscal year during 2012. Previously, the fiscal year was based on the solar calendar and ran March 21 through March 20 (in most years). Starting in 2012, the fiscal year will end on December 21 (in most years), aligning it more closely with the Gregorian calendar. The authorities hope that this will facilitate development budget implementation by moving the last quarter during which most expenditure takes place to one with more conducive weather conditions. 2012 as the transition year will have only 9 months, March through December.

${ }^{6}$ For the full calendar year, revenue collection in 2012 would be 11.3 percent of GDP compared to 11.0 percent of GDP in 2011.
} 


\section{B. Safeguarding the Financial Sector}

\section{The authorities are concerned about the high number of structurally weak}

banks in the system. 8 out of 12 domestic banks have a CAMEL rating of 4 or lower. ${ }^{7} \mathrm{~A}$ number of these banks are expected to not meet the increase in the regulatory minimum paidup capital to US\$20 million by June 2012 from the current US\$10 million. The authorities intend to either close or merge those banks that do not meet the new minimum paid-up capital (structural benchmark, MEFP paragraph 23). Subsequently, they will review the remaining domestic banks and assess whether they are on a path of improvement, or whether there is a need to close or merge additional banks.

\section{The new banking law will help strengthen the system. The new, consolidated} banking law, once enacted, will help prevent, mitigate, and effectively respond to the problems in the financial sector. ${ }^{8}$ The revisions strengthen corporate governance provisions, regulate capital requirements, large exposures, and related parties, as well as enhance supervision and bank resolution (MEFP paragraph 21). Under the program the authorities committed to submitting the draft law to parliament by end-September 2012 (structural benchmark), followed by adapting central bank regulations accordingly, and preparing informational circulars and trainings by end-December 2012 (structural benchmark). To supplement these efforts and improve the contract enforcement infrastructure, the authorities will also establish, by end-December 2012, a training program for court officials to create capacity in relevant banking laws and commercial codes.

\section{The authorities' program further strengthens banking supervision (MEFP} paragraph 22). This will be done through administrative improvements, such as reviewing and strengthening of enforcement regulations, improving the timeliness of enforcement actions, and the monitoring of the outstanding enforcement orders. In addition, the central bank will hire additional staff, provide more training in risk-based banking supervision, implement a new organizational structure for the Financial Supervision Department (structural benchmark), and change the mandate of the Risk Management Department (to focus on risks within DAB only). In addition, DAB intends to strengthen the legal protection of supervisory staff from rogue, corrupt or politically motivated elements, in good faith conduct of duties.

\footnotetext{
${ }^{7}$ The 12 banks include New Kabul Bank, which has not yet been rated.

${ }^{8}$ In 2011 the Fund advised on drafting the previous version of draft amendments to the banking law and also, separately, provided extensive advice on the earlier version of a separate Islamic Banking Law. The authorities are currently merging these two laws so that a consolidated banking law comprehensively covers allconventional and Islamic - banking provisions and products. The new banking law will have to be reviewed by the Supreme Council of the central bank and the ministry of justice before it is submitted to parliament. Fund staff will be further consulted.
} 


\section{The authorities also intend to strengthen the central bank capitalization}

framework. They will implement the provisions of the recently signed memorandum of understanding, including through changes in the law where necessary - to be finalized by December 2012 and sent to parliament by June 2013 (structural benchmark, MEFP paragraph 31). A recapitalization, if needed, will be effected no later than 2014. Going forward, to fully regularize the financial relations between the central bank and the ministry of finance, a service agreement between the parties should mandate the central bank paying market interest rates on government deposits and the ministry paying market or cost based fee for services rendered to it by the bank.

\section{Reflecting delays in appointing a privatization advisor, offering New Kabul} Bank for sale will take longer than anticipated. The economic committee of the cabinet has approved the sale of New Kabul Bank on February 21, 2012, and the privatization advisor, financed by DfID, started his work in May. Developing the privatization plan will take until August 2012 (structural benchmark), and the bank can then be offered for sale by end-September (structural benchmark), and not in June 2012 as previously envisaged under the program (MEFP paragraph 25). If there is no interest from a suitable buyer or the sale fails, the authorities plan to wind down the bank by end-December 2013-taking longer than previously expected because handing over the salary payment function to other banks as well as migrating the existing deposit accounts to viable financial institutions capable of accommodating them is likely to take more time. Over 400,000 government employees receive their salaries by direct deposit to their bank accounts—-largely at New Kabul Bank. The recently awarded new salary payment contract — to Azizi Bank and new Kabul Bankwill allow an additional bank to start expanding coverage and building capacity in this field. The authorities note that civil servants will be able to freely choose the bank in which their salaries are paid once the new national payments systems (real time gross settlement, automated clearing house, and a national switch), financed by the World Bank, will be operational in mid-2014.

\section{Strengthening Economic Governance}

29. The authorities have made a high-level commitment to tackle the deficiencies in their AML/CFT framework in line with the targeted review process of the Financial Action Task Force International Cooperation Review Group (MEFP paragraph 30). In May, the authorities met with the Regional Review Group and made a ministerial level commitment to an action plan designed to bring them into compliance with FATF recommendations. The action plan will be agreed with the Financial Action Task Force in June 2012. The authorities agreed to implement the elements of the action plan according to a timeline discussed and agreed in May (structural benchmark). In this context, they will also strengthen information sharing among the various entities involved in AML/CFT (structural benchmark).

30. Following up on their economic crimes strategy, the authorities will establish a task force to steer and oversee work in this area. The economic crimes task force will be 
led by a senior official and, for example, oversee the passage and implementation of new laws, and function as a coordinating body for the various entities concerned with economic crimes (structural benchmark, MEFP paragraph 29).

\section{Moving Toward Fiscal Sustainability}

31. The authorities stressed the importance of the VAT in their strategy for revenue generation and fiscal sustainability. In line with the objective of introducing a VAT in 2014, the draft law will be sent to cabinet for discussion by September 2012, and to parliament for approval by end-December 2012 (structural benchmark, MEFP paragraph 34). In the meantime, the authorities will prepare for the VAT implementation by: (i) identifying large taxpayers who are not currently filing with the Large Taxpayer Office (LTO) - but should do so based on their turnover - with a view to transferring them to the LTO;

(ii) revisiting the LTO threshold so as to optimize the number of businesses in the office; and (iii) strengthening office's capacity through internal staff transfers and trainings (structural benchmark, MEFP paragraph 35).

32. The authorities are putting in place a fiscal regime for natural resources. The objective is to attract investment and ensure that the government has a reasonable share of the economic rent (MEFP paragraphs 36 and 37). Importantly, they are committed to channeling revenues transparently through the budgetary process, without earmarking or offbudget spending. To that end, by June 2013, they intend to finalize amendments to the appropriate laws that create an effective and transparent fiscal regime for natural resources in full compliance with the Extractive Industries Transparency Initiative and in consultation with the IMF and the World Bank (structural benchmark).

\section{The authorities regret that it will take more time to work on the law on}

marketable sukuk securities. Sukuks are intended mainly to improve cash management; the authorities noted that they did not envisage using them to finance future budget deficits. The ministry of finance is finalizing the draft law, with a view to submitting it to the ministry of justice by September 2012 (structural benchmark) and to the parliament by March 2013 (MEFP paragraph 39). This is later than initially planned (sending the law to parliament by September 2012) to allow for wide consultations with all stakeholders and ensure that the law is fully consistent within the Afghan legal framework. In the meantime, a sukuk implementation plan with specific actions and a timetable will be prepared for the actual issuance, intended for late 2013 or early 2014.

\section{E. Fiscal and External Debt Sustainability Analysis ${ }^{9}$}

\section{The authorities will seek substantial and prolonged financial support from} donors at the forthcoming Tokyo Conference. Afghanistan's financing needs will decline

\footnotetext{
${ }^{9}$ See Islamic Republic of Afghanistan, Joint World Bank/IMF Debt Sustainability Analysis Update below.
} 
from about 47 percent of GDP at present (including off-budget development and operating spending paid for directly by donors) to just over 20 percent of GDP by 2020. Based on Fund and World Bank staff estimates, Afghanistan's financing needs will remain at this elevated level for some time. If the Tokyo Conference does not generate the hoped-for support, staff advises that the authorities build up spending very carefully and only in line with their sustainable resource envelope. This will require very difficult choices and could imply that Afghanistan's development ambitions will take longer to realize. Fund staff is developing a model-based framework that will allow quantifying the macroeconomic effects of the decline in the military presence and aid, and illustrate policy trade-offs.

\section{Expenditure pressures come from three sources: security, development and the} take-over of the recurrent costs implications of donor projects. First, security spending needs are projected to be high — stabilizing at about 15 percent of GDP after 2020 compared with other low-income countries..$^{10}$ Second, staff believes that development spending should stabilize at about 10 percent of GDP. The authorities would prefer to frontload investment until 2020 to reach 16 percent of GDP on average but decline to below 10 percent of GDP thereafter. However, given likely resource constraints and limited implementation capacity, it is unlikely that such a high level of development spending can be achieved. Third, the budget will have to accommodate the recurrent costs of projects currently financed by donors of about $4 \frac{1}{2}$ percent of GDP. Taken together, government expenditures could reach close to 40 percent of GDP in 2025, a level much higher than what governments in comparable countries would spend.

\section{Domestic revenues would barely cover half of projected government spending.}

Successful implementation of a VAT in 2014 should generate about 2 percent of GDP, while an excise tax (not yet a part of the program, but assumed for 2018), could yield an additional 1 percent of GDP. Furthermore, under a cautiously optimistic scenario, mining revenues could yield about 2 percent of GDP by 2025 (the authorities hope that mining will yield about 3 percent of GDP). Taken together, domestic revenue could increase to about 17 percent of GDP by 2020, from today's 11 percent of GDP.

\section{With these trends, the government will continue to run large fiscal deficits of about 20-22 percent of GDP in the long run. Only a fraction of this amount can be} financed by external concessional loans or through issuance of domestic debt. Under these circumstances, fiscal sustainability (defined as domestic revenues covering operating expenditures) falls beyond the projections' horizon. Without high and prolonged donor support, the proposed strategy is not viable.

\footnotetext{
${ }^{10}$ Security cost projections are based on ISAF estimates. Most of the projected reduction is expected to take place during 2015-17, reflecting the envisaged managed troop reduction from 352,000 to 228,500. The authorities have not yet committed to this reduction, and keeping the troop size at 352,000 would carry a price tag of about US $\$ 700$ million in 2017 or $2 \frac{1}{2}$ percent of GDP.
} 
38. As a result, Afghanistan remains at high risk of debt distress even after the HIPC completion point and delivery of debt relief under the MDRI. External public and publicly guaranteed debt amounted to US\$1.2 billion, or 7 percent of GDP, in 2011, most of which was owed to multilateral creditors. ${ }^{11}$ Under the baseline scenario of the debt sustainability analysis, the present value of public external debt would reach about 5 percent of GDP by the end of the projection period, below the indicative debt-burden threshold applying to a country like Afghanistan. ${ }^{12}$ However, should donors decide to reduce aid rapidly or provide loans rather than grants, or should security fail to stabilize, or structural reforms and governance improvements lag, Afghanistan's debt burden would become unsustainable. Risks to the outlook are tilted to the downside, linked to security prospects, the strength of future reforms, and a possible deterioration of the fiscal outlook with more limited access to grants.

\section{Program Modalities}

39. The program is fully financed and access remains appropriate. Donor inflows, on which Afghanistan is highly dependent, can be uncertain and volatile, thus calling for a sufficiently robust level of foreign reserves. The proposed access should be sufficient to cover any residual financing gaps and contribute to reserves equivalent to about seven months of imports.

40. The authorities request a waiver and some program design modifications.

- Waiver of nonobservance. The slippage in revenue collection is minor (less than 0.2 percent of GDP) and, given that it reflects trade disruptions at the border with Pakistan, temporary and beyond authorities' control. In staff's view, the slippage does not jeopardize successful implementation of the program. In addition, the authorities have taken immediate remedial measures to strengthen revenue collection: specifically, they committed to phasing out all existing concessions for certain taxpayer groups that have hitherto undermined the revenue base (MEFP paragraph 35).

- Modification of quantitative performance criteria for end-September 2012. The request reflects changing circumstances and new information. In fact, two of the three performance criteria proposed for modification entail some tightening. The third one, the ceiling on reserve money, needs to be adjusted to accommodate a change in seasonality related to a shift in the fiscal year.

\footnotetext{
${ }^{11}$ After debt relief under HIPC and MDRI, as well as from Paris Club creditors. Negotiations with the one remaining Paris Club creditor (Russia) on the delivery of its debt relief commitments continue, as well as with other creditors on comparable terms.

${ }^{12}$ Afghanistan is classified as a "weak performer" and its thresholds therefore: 30 percent of NPV for the debtto-GDP ratio; 100 percent of NPV for the debt-to-exports ratio; 200 percent for the debt-to-revenue ratio; 15 percent for the debt service-to-exports ratio; and 20 percent for the debt service-to-revenue ratio.
} 
- Modification of benchmarks for the second review. The authorities believe that more time is needed to complete the structural benchmark on offering New Kabul Bank for sale and submitting the sukuk law to parliament. They have introduced intermediate steps to document progress toward these important objectives.

41. The authorities are requesting a rephasing of the arrangement, including a change to the schedule of reviews, in order to align it with the new fiscal year. In 2012 the government is changing its fiscal year (Footnote 5), and asks to align the review cycle with the new fiscal year. Through 2011/12, the fiscal year corresponded to the Afghan calendar year (based on the solar year) running from March 21 to March 20 in most years. Starting in 2013, the fiscal year will run from December 22 to December 21 in most years. 2012 will be a transition year with only nine months in the fiscal year (March-December). Accordingly, the schedule of test dates and reviews, starting with the third review, is brought forward by three months (implying an interval of three months between the second and third review), with a corresponding change to the schedule of disbursements. Thus, the second, third and fourth reviews of the program would be based on performance through September 2012, December 2012 and June 2013, respectively - the quantitative performance criteria and structural benchmarks have been set accordingly. The conditionality under the third and fourth review will be revisited at the time of the second review.

\section{The authorities and staff agreed to clarify some definitions in the technical} memorandum of understanding. The clarifications include the definitions of reserve money, net central bank credit to the government, two adjustors and the treatment of treasury's IMF accounts. These changes are for clarification only, and do not bring any material changes to the design of the program, including to the quantitative performance criteria.

43. An update safeguards assessment of the central bank was finalized in December 2011. It found that while most of the previous safeguards recommendations had been implemented, an effective internal audit mechanism had still not been established and governance oversight was weak. The assessment also made recommendations to address risks emerging as a result of the Kabul Bank crisis including with respect to central bank autonomy. Since the assessment, some recommendations have been implemented, albeit with delay. The central bank is committed to implementing the remaining safeguards recommendations, with priority assigned to development of the internal audit function (with external support) and strengthening of Audit Committee oversight.

44. Economic data are adequate for program monitoring and surveillance, but there are significant shortcomings. The quality and timeliness of monetary and fiscal data are broadly adequate, although coverage is still deficient. Other data also suffer from weaknesses, especially data on prices, national accounts, balance of payments, and social indicators. Fund technical assistance is being provided to strengthen statistical data systems. It will be useful to prepare a Report on the Observance Standards and Codes on data to help develop an economic statistics plan. 


\section{Staff Appraisal}

\section{The authorities have made notable progress in a number of important reform} areas. The ministry of finance has sent a draft VAT law to the ministry of justice for review and rolled out the new border management model to two additional crossings. DAB and the ministry of finance have agreed on the central bank's capitalization framework. And, the government has included its new economic crimes strategy in its NPPs.

\section{Notwithstanding this, program implementation has been weak and needs to} improve for the second review. While the individual missed quantitative targets or delays in structural benchmarks can all be explained, the fact remains that only a select few measures have been implemented as described in the authorities' program request. Going forward, additional delays have been flagged, and more could occur. This raises questions over the authorities' capacity to implement a program and ownership in the government at large. For a successful second review, reform implementation has to become more timely and in line with the commitments' spirit.

\section{Asset recovery from Kabul Bank has been disappointing despite the boost over} the last eight weeks after the Presidential Decree. One and a half years after the failure of Kabul Bank, cash recoveries have been low, including because the receiver did not have sufficient capacity to follow up on nonpayments under the previously existing legally binding agreements. With the Presidential Decree, cash recoveries have picked up, and a special tribunal is now considering the cases of the two main shareholders, while the Financial Dispute Resolution Committee will deal with the remaining shareholders and related parties. Staff has concerns over the quality of the process that has been initiated, and encourages the authorities to send a strong signal that the rule of law holds in Afghanistan. Failing that, there is a risk that asset recovery going forward may be fairly limited. The authorities should also report quarterly to the public on their asset recovery progress starting in September 2012, and take the necessary actions that will provide the environment in which seized properties in Afghanistan can be sold at a reasonable price.

\section{The authorities' program for the coming year is appropriately focused on three} areas. First, financial sector weaknesses constitute vulnerabilities and can be costly, as evidenced by the Kabul Bank failure. The supervisor should ensure that all banks have adequate capital that is commensurate with their risk exposure. More generally, banking sector weaknesses are part of the reason why the sector is not playing a role in facilitating private sector led growth. The sale of New Kabul Bank could be an important milestone for financial sector development, but only if a proper investor is identified. If no suitable investor steps forward, the bank should be wound down as quickly as possible. Second, poor economic governance is a macroeconomic risk that could derail the authorities' growth and development objectives. And third, given Afghanistan's security and development spending requirements, the authorities need to act decisively to strengthen domestic revenue mobilization, including by introducing a VAT and putting in place a sound fiscal regime for the natural resource sector. 
49. The authorities' development objectives over the coming decade are very ambitious. The NATO summit has generated significant pledges, but has also sent a signal that donors are unwilling to make indefinite commitments. Even with the planned domestic revenue efforts, the targeted spending level implies large financing needs beyond the projection horizon. The outcome of the Tokyo Conference will determine how ambitious the authorities can be in their development agenda. If pledges fall short of the proposed financing needs, the authorities need to constrain spending to within the available resource envelope or find additional ways to raise domestic revenues. Afghanistan has only very limited scope for debt financing, and cannot afford to build up expenditure expectations that cannot be delivered.

50. The program is subject to significant risks. First, powerful vested interests have the means and the potential to paralyze political will and obstruct reforms. As these vested interests may ultimately put into question Afghanistan's political and economic viability, it is imperative that the government stand firm on its governance and rule of law agenda and fully apply Afghan law. Second, the reduction in international presence, including withdrawal of combat troops and decrease in donor activity could have more severe than expected economic repercussions, leading to fiscal and external pressures. Third, deterioration in the security situation would reduce growth and make it impossible to implement reforms.

\section{Despite the delays and implementation difficulties, staff recommends the} completion of the first review. The reform agenda is ambitious, given implementation capacity. In this context, the Fund program is an effective mechanism to generate support for the reform agenda and maintain momentum for the difficult measures needed to create an environment for high and inclusive growth. The program also acts as a catalyst for donor support, with some donors explicitly linking their support to the program. Staff supports the request for a waiver of nonobservance for the end-March 2012 performance criterion on central government revenue collection, given that the authorities are taking corrective action. Lastly, staff also supports the requested modification of September 2012 performance criteria and the rephasing of disbursements. 
Figure 1. Afghanistan: Real Sector
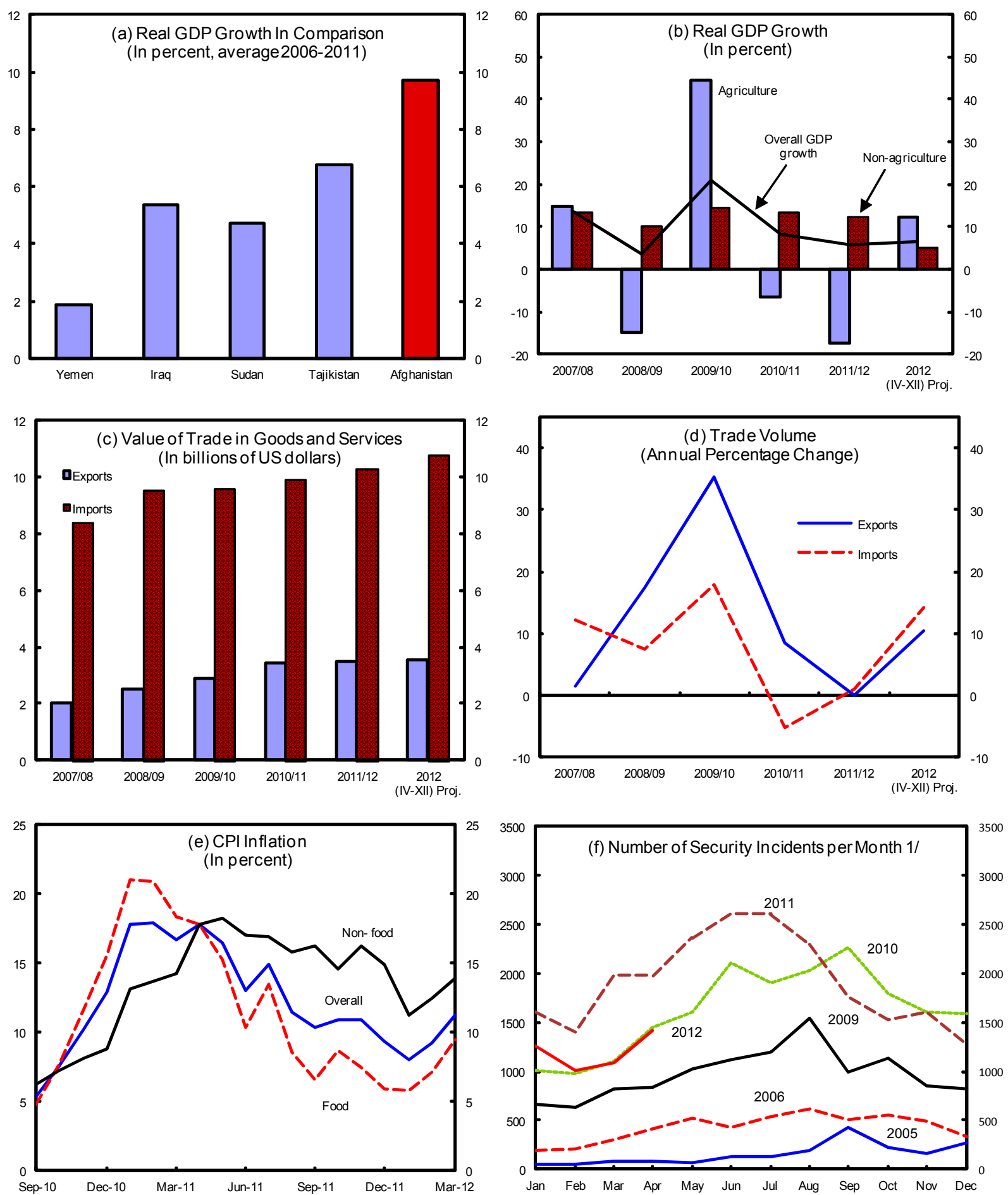

Sources: Afghan authorities; IMF, WEO; United Nations Department of Safety and Security; and Fund staff estimates.

1/ Attacks and serious beating incidents perpetrated by anti-government elements throughout Afg hanistan. They include suicide and stand off attacks; bombings; attacks on district centers, aid and military convoys and their contractors; night letters; assasinations; illegal check points and beatings. 
Figure 2. Afghanistan: Fiscal Sector
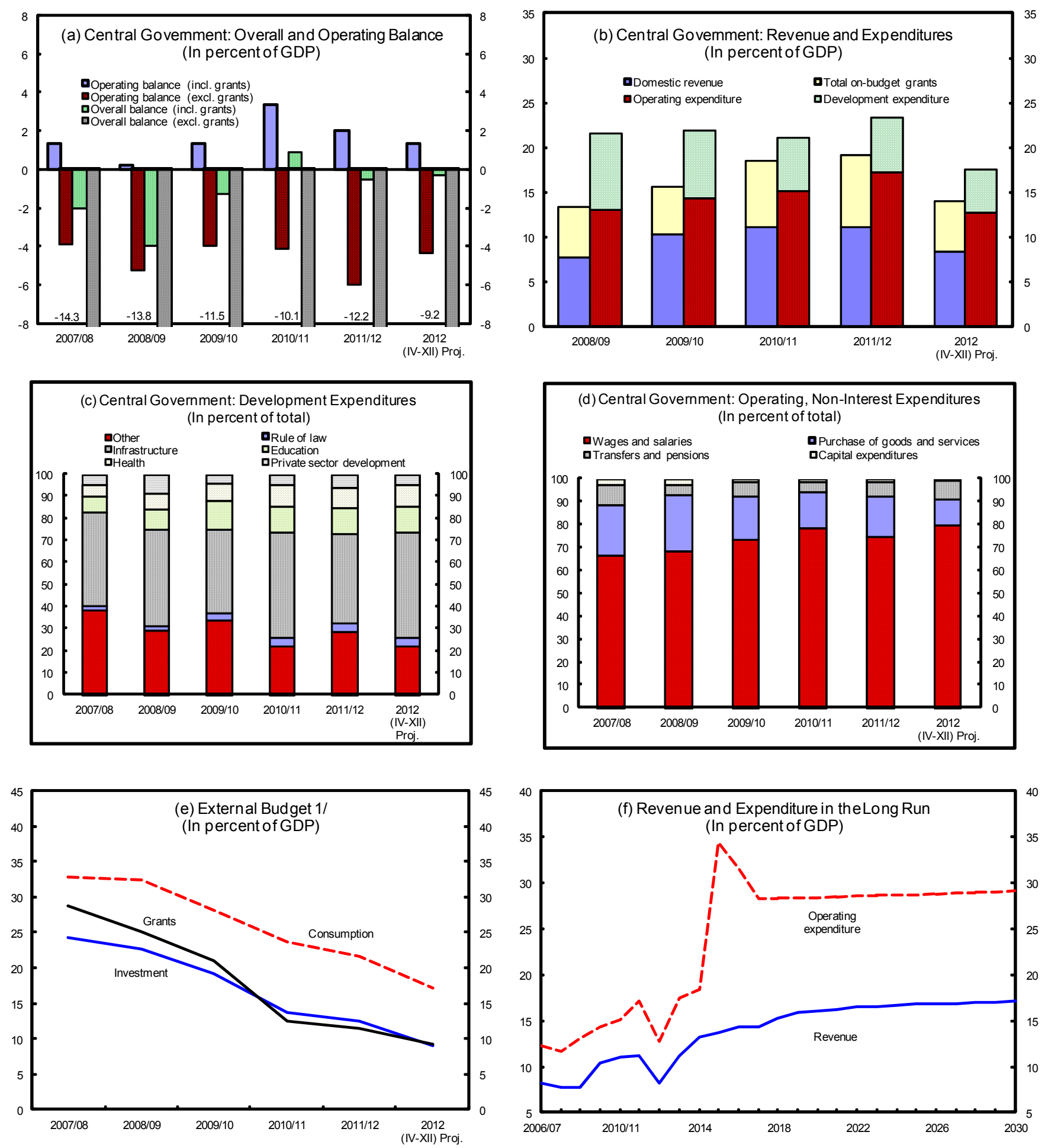

Sources: Afg han authorities; and Fund staff estimates.

1/ Estimated activity off-budget by international community. 
Figure 3. Afghanistan: Monetary Sector
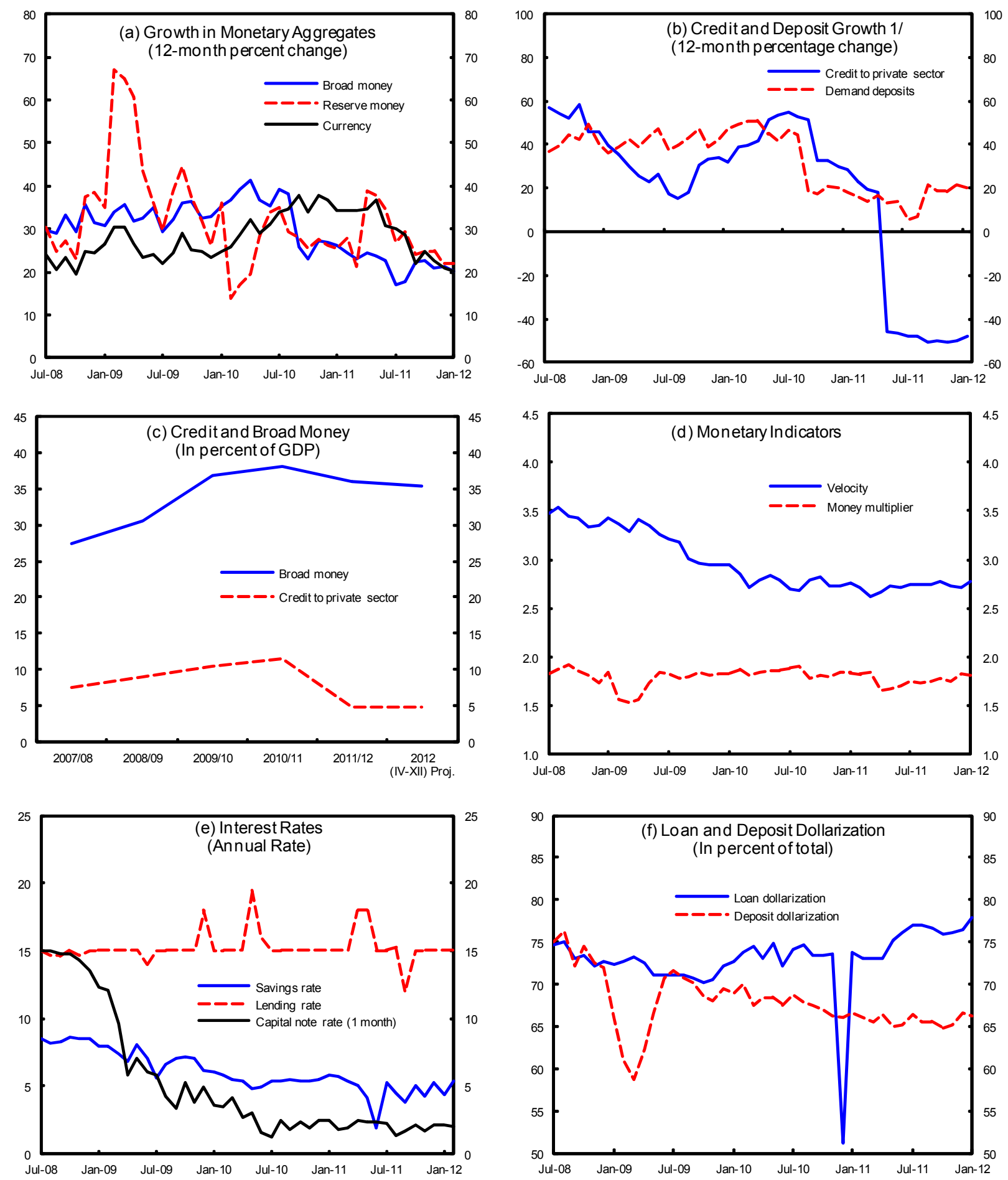

Sources: Afg han auth orities; and Fund staff estimates.

1/ The sharp drop in credit to the private sector reflects the write-off of Kabul Bank loans. 
Figure 4. Afghanistan: External Sector
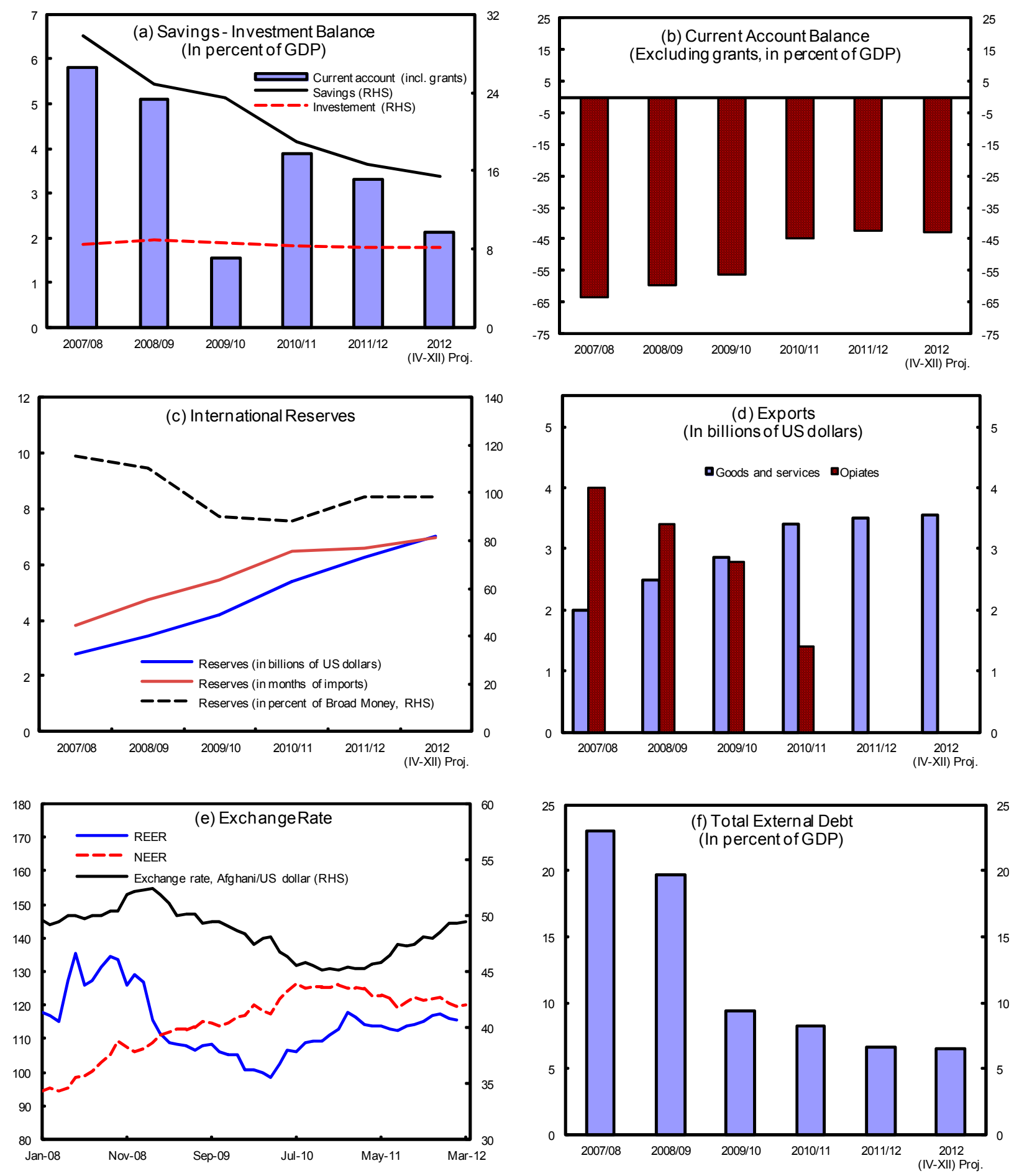

Sources: Afg han auth orities; United Nations Office on Drugs and Crime; and Fund staff estimates. 
Table 1. Islamic Republic of Afghanistan: Selected Economic Indicators, 2010-2014

(Quota: SDR 161.9 million)

(Population: approx. 31 million)

(Per capita GDP: approx. US\$580; 2011)

(Poverty rate: 36 percent; 2008 )

(Main export: opium, US\$2.8 billion; carpets, US\$155 million; 2010)

\begin{tabular}{|c|c|c|c|c|c|c|c|}
\hline & \multicolumn{3}{|c|}{ Afghan calendar year $1 /$} & \multicolumn{4}{|c|}{ Year 1/ } \\
\hline & \multirow{2}{*}{$\begin{array}{r}2010 / 11 \\
\text { Act. }\end{array}$} & \multicolumn{2}{|c|}{$2011 / 12$} & \multirow{2}{*}{$\begin{array}{c}2011 \\
\text { Est. }\end{array}$} & \multirow{2}{*}{$\begin{array}{l}2012 \\
\text { Proj. }\end{array}$} & \multirow{2}{*}{$\begin{array}{l}2013 \\
\text { Proj. }\end{array}$} & \multirow{2}{*}{$\begin{array}{r}2014 \\
\text { Proj. }\end{array}$} \\
\hline & & Prog. & Est. & & & & \\
\hline Output and prices 2/ & \multicolumn{7}{|c|}{ (Annual percentage change, unless otherwise indicated) } \\
\hline Real GDP & 8.4 & 5.7 & 5.8 & 5.8 & 6.9 & 6.5 & 5.9 \\
\hline Nominal GDP (in billions of Afghanis) & 730 & 862 & 875 & 846 & 1,003 & 1,140 & 1,270 \\
\hline Nominal GDP (in billions of U.S. dollars) & 15.9 & 18.4 & 18.3 & 18.1 & 20.0 & 22.0 & 23.7 \\
\hline Food & 7.3 & 8.1 & 9.5 & 12.5 & 7.2 & 5.4 & $\ldots$ \\
\hline Non-food & 8.3 & 14.2 & 15.3 & 15.7 & 11.7 & 8.5 & $\ldots$ \\
\hline Consumer prices (end of period) & 16.6 & 7.7 & 11.3 & 9.4 & 7.5 & 5.4 & 5.0 \\
\hline Investment and savings & \multicolumn{7}{|c|}{ (In percent of GDP) } \\
\hline Gross domestic investment & 26.5 & 22.6 & 25.3 & 25.3 & 25.0 & 25.0 & 23.5 \\
\hline Of which: Private & 8.4 & 8.6 & 8.2 & 8.2 & 8.2 & 9.3 & 10.5 \\
\hline Gross domestic savings & 30.4 & 22.6 & 28.7 & 28.7 & 26.7 & 25.6 & 22.7 \\
\hline \multicolumn{8}{|l|}{ Public finances (central government) } \\
\hline Domestic revenues and grants & 22.0 & 23.7 & 22.7 & 21.0 & 23.2 & 23.4 & 25.4 \\
\hline Domestic revenues & 11.0 & 11.5 & 11.1 & 10.9 & 11.3 & 11.3 & 13.4 \\
\hline Grants & 11.0 & 12.2 & 11.6 & 10.2 & 11.9 & 12.1 & 12.0 \\
\hline Expenditures & 21.1 & 23.8 & 23.3 & 21.7 & 24.3 & 24.1 & 25.2 \\
\hline Operating $3 /$ & 15.1 & 17.6 & 17.2 & 16.1 & 18.2 & 17.7 & 18.6 \\
\hline Development & 6.0 & 6.2 & 6.1 & 5.6 & 6.1 & 6.4 & 6.6 \\
\hline Operating balance (excluding grants) 4/ & -4.1 & -6.1 & -6.1 & -5.2 & -6.9 & -6.4 & -5.2 \\
\hline Overall balance (including grants) & 0.9 & 0.0 & -0.6 & -0.6 & -1.1 & -0.8 & 0.2 \\
\hline Public debt & 8.2 & $\ldots$ & 6.6 & 6.9 & 6.7 & 6.6 & 6.9 \\
\hline Monetary sector & \multicolumn{7}{|c|}{ (Annual percentage change, end of period, unless otherwise indicated) } \\
\hline Reserve money & 21.3 & 18.8 & 16.1 & 21.9 & 18.7 & 16.0 & $\ldots$ \\
\hline Currency in circulation & 34.2 & 17.8 & 11.9 & 20.8 & 18.7 & 15.8 & $\ldots$ \\
\hline Broad money & 23.1 & 18.3 & 12.7 & 21.3 & 14.5 & 15.4 & $\ldots$ \\
\hline Interest rate, 28-day capital note (in percent) & 2.4 & $\cdots$ & 2.0 & 2.1 & $\cdots$ & $\cdots$ & $\cdots$ \\
\hline Imports of goods (in U.S. dollars, percentage change) & 4.5 & 0.4 & 7.4 & $\ldots$ & 14.3 & 4.6 & 0.8 \\
\hline Merchandise trade balance & -44.4 & -34.1 & -42.2 & -40.6 & -44.4 & -43.2 & -39.6 \\
\hline \multicolumn{8}{|l|}{ Current account balance } \\
\hline Excluding official transfers & -44.6 & -34.3 & -42.3 & -40.7 & -44.6 & -43.0 & -39.6 \\
\hline Including official transfers & 3.9 & 0.1 & 3.3 & 3.6 & 1.7 & 0.5 & -0.8 \\
\hline Foreign direct investment & 2.0 & 2.1 & 1.7 & 1.7 & 1.6 & 2.7 & 3.4 \\
\hline Total external debt 6/ & 8.2 & $\ldots$ & 6.6 & 6.9 & 6.7 & 6.6 & 6.7 \\
\hline Gross international reserves (in millions of U.S. dollars) & 5,403 & 6,121 & 6,234 & 6,208 & 6,721 & 7,286 & 7,750 \\
\hline (Import coverage) $7 /$ & 6.4 & $\ldots$ & 6.5 & 6.7 & 6.8 & 7.3 & 7.7 \\
\hline (Relative to external debt service due) & 435 & 425 & 468 & 474 & 421 & 181 & 65 \\
\hline Afghanis per U.S. dollar (average) & 45.8 & $\ldots$ & 47.8 & 46.7 & $\ldots$ & $\ldots$ & $\ldots$ \\
\hline Real effective exchange rate (average, percentage change) & 3.8 & $\cdots$ & 5.1 & 8.9 & $\cdots$ & $\cdots$ & $\cdots$ \\
\hline \multicolumn{8}{|l|}{ Memorandum items: } \\
\hline \multicolumn{8}{|l|}{ Opium statistics (dry opium) } \\
\hline Production (in tons) & 3,600 & $\ldots$ & 5,800 & 5,800 & $\ldots$ & $\ldots$ & $\ldots$ \\
\hline Price (in U.S. dollars per kilogram) & 169 & $\ldots$ & 241 & 241 & $\ldots$ & $\ldots$ & ... \\
\hline
\end{tabular}

Source: Afghan authorities; United Nations Office on Drugs and Crime; and Fund staff estimates and projections.

$1 /$ Through 2011/12, the fiscal year corresponded to the Afghan calendar year, which is based on the solar year running from March 21 to March 20 , in most years. In 2012, the authorities are transitioning to a fiscal year that is closer aligned with the Gregorian calendar year and runs December 22 - December 21, in most years. (Accordingly, the interim FY2012 has only 9 months.) In this table, 2011 and 2012 are recalculated to full years for comparison.

2/ Excluding the narcotics economy.

3/ Comprising mainly current spending.

4/ Defined as domestic revenues minus operating expenditures.

5/ Includes official recorded exports, estimates of smuggling, reexports and sales to nonresidents.

6/ After HIPC and MDRI debt relief, as well as debt relief beyond HIPC relief from Paris Club creditors.

7/ In months of next year's imports of goods and services, excluding imports for reexport. 
Table 2. Islamic Republic of Afghanistan: Medium- and Long-Term Macroeconomic Framework, 2011-2025

\begin{tabular}{|c|c|c|c|c|c|c|c|c|c|c|c|c|c|c|c|}
\hline & $\begin{array}{r}2011 \\
\text { Est. }\end{array}$ & $\begin{array}{l}2012 \\
\text { Proj. }\end{array}$ & $\begin{array}{l}2013 \\
\text { Proj. }\end{array}$ & $\begin{array}{c}2014 \\
\text { Proj. }\end{array}$ & $\begin{array}{l}2015 \\
\text { Proj. }\end{array}$ & $\begin{array}{l}2016 \\
\text { Proj. }\end{array}$ & $\begin{array}{l}2017 \\
\text { Proj. }\end{array}$ & $\begin{array}{l}2018 \\
\text { Proj. }\end{array}$ & $\begin{array}{l}2019 \\
\text { Proj. }\end{array}$ & $\begin{array}{l}2020 \\
\text { Proj. }\end{array}$ & $\begin{array}{c}2021 \\
\text { Proj. }\end{array}$ & $\begin{array}{c}2022 \\
\text { Proj. }\end{array}$ & $\begin{array}{l}2023 \\
\text { Proj. }\end{array}$ & $\begin{array}{c}2024 \\
\text { Proj. }\end{array}$ & $\begin{array}{r}2025 \\
\text { Proj. }\end{array}$ \\
\hline Output and prices $1 /$ & \multicolumn{15}{|c|}{ (Annual percentage change, unless otherwise indicated) } \\
\hline Real GDP & 5.8 & 6.9 & 6.5 & 5.9 & 5.9 & 5.3 & 4.2 & 4.4 & 5.8 & 6.0 & 4.4 & 4.4 & 4.5 & 4.0 & 4.0 \\
\hline Nominal GDP (in billions of U.S. dollars) & 18.1 & 20.0 & 22.0 & 23.7 & 25.4 & 27.0 & 28.4 & 30.0 & 32.0 & 34.2 & 36.1 & 38.2 & 40.3 & 42.4 & 44.7 \\
\hline GDP per capita (in U.S. dollars) & 587 & 629 & 673 & 704 & 732 & 756 & 772 & 790 & 820 & 852 & 875 & 901 & 928 & 953 & 979 \\
\hline Consumer prices (period average) & 13.8 & 9.1 & 6.7 & 5.0 & 5.0 & 5.0 & 5.0 & 5.0 & 5.0 & 5.0 & 5.0 & 5.0 & 5.0 & 5.0 & 5.0 \\
\hline Investment and savings & \multicolumn{15}{|c|}{ (In percent of GDP, unless otherwise indicated) } \\
\hline Gross domestic investment & 25.3 & 25.0 & 25.0 & 23.5 & 20.1 & 19.6 & 20.4 & 20.3 & 20.6 & 21.6 & 21.2 & 20.9 & 20.8 & 20.5 & 20.5 \\
\hline Of which: Private & 8.2 & 8.2 & 9.3 & 10.5 & 11.0 & 11.9 & 12.3 & 12.6 & 13.6 & 14.4 & 14.0 & 13.5 & 13.4 & 13.0 & 13.0 \\
\hline Gross domestic savings & 28.7 & 26.7 & 25.6 & 22.7 & 16.9 & 15.8 & 16.6 & 16.7 & 17.8 & 18.1 & 18.4 & 18.4 & 18.3 & 18.3 & 18.4 \\
\hline Of which: Private & 12.1 & 11.1 & 10.5 & 9.4 & 8.7 & 9.4 & 9.4 & 9.7 & 11.2 & 11.3 & 11.7 & 11.4 & 11.5 & 11.5 & 11.7 \\
\hline \multicolumn{16}{|l|}{ Public finances } \\
\hline Domestic revenues and grants & 21.0 & 23.2 & 23.4 & 13.4 & 14.0 & 14.5 & 14.5 & 15.6 & 16.1 & 16.2 & 16.5 & 16.7 & 16.7 & 16.9 & 17.0 \\
\hline Domestic revenues & 10.9 & 11.3 & 11.3 & 13.4 & 14.0 & 14.5 & 14.5 & 15.6 & 16.1 & 16.2 & 16.5 & 16.7 & 16.7 & 16.9 & 17.0 \\
\hline Grants & 10.2 & 11.9 & 12.1 & $\ldots$ & $\ldots$ & $\ldots$ & $\ldots$ & $\ldots$ & $\ldots$ & $\ldots$ & $\ldots$ & $\ldots$ & $\ldots$ & $\ldots$ & \\
\hline Expenditures & 21.7 & 24.3 & 24.1 & 25.2 & 41.8 & 38.8 & 35.7 & 36.0 & 36.2 & 36.3 & 36.6 & 36.9 & 36.9 & 36.9 & 37.0 \\
\hline Operating 2/ & 16.1 & 18.2 & 17.7 & 18.6 & 35.1 & 31.9 & 28.7 & 28.8 & 28.7 & 28.7 & 28.8 & 29.0 & 28.9 & 29.0 & 29.0 \\
\hline Development & 5.6 & 6.1 & 6.4 & 6.6 & 6.7 & 6.9 & 7.1 & 7.2 & 7.4 & 7.6 & 7.8 & 8.0 & 8.0 & 8.0 & 8.0 \\
\hline Operating balance (excluding grants) $3 /$ & -5.2 & -6.9 & -6.4 & -5.2 & -21.1 & -17.4 & -14.1 & -13.2 & -12.7 & -12.5 & -12.4 & -12.3 & -12.2 & -12.1 & -12.0 \\
\hline Overall budget balance (including grants) & -0.6 & -1.1 & -0.8 & -11.8 & -27.8 & -24.3 & -21.2 & -20.5 & -20.1 & -20.1 & -20.2 & -20.3 & -20.2 & -20.0 & -20.0 \\
\hline \multicolumn{16}{|l|}{ External sector $1 /$} \\
\hline Merchandise trade balance & -40.6 & -44.4 & -43.2 & -39.6 & -37.0 & -30.7 & -27.9 & -26.0 & -23.2 & -23.7 & -22.5 & -22.1 & -22.0 & -21.7 & -21.5 \\
\hline Current account balance, excluding grants & -40.7 & -44.6 & -43.0 & -39.6 & -36.8 & -30.9 & -28.4 & -26.9 & -24.7 & -25.4 & -24.4 & -24.2 & -24.0 & -23.4 & -23.1 \\
\hline Gross reserves (in millions of U.S. dollars) & 6,208 & 6,721 & 7,286 & 7,750 & 7,750 & 8,130 & 8,070 & 7,950 & 7,950 & 8,030 & 8,040 & 8,070 & 8,080 & 8,090 & 8,120 \\
\hline Import coverage 4/ & 6.7 & 6.8 & 7.3 & 7.7 & 8.0 & 8.3 & 7.9 & 7.3 & 6.6 & 6.3 & 5.8 & 5.6 & 5.3 & 5.1 & 4.9 \\
\hline \multicolumn{16}{|l|}{ Memorandum items } \\
\hline External budget grants $5 /$ & 34.1 & 34.4 & 31.5 & 26.8 & 6.7 & 4.2 & 4.4 & 3.6 & 2.4 & 2.2 & 2.0 & 2.0 & 2.0 & 2.0 & 2.0 \\
\hline Total external debt $6 /$ & 6.9 & 6.7 & 6.6 & 6.7 & 6.7 & 6.7 & 6.8 & 6.8 & 6.8 & 6.8 & 6.9 & 7.0 & 7.1 & 7.3 & 7.5 \\
\hline Total public debt 6/ & 6.9 & 6.7 & 6.6 & 6.9 & 7.1 & 7.8 & 7.8 & 8.3 & 8.5 & 8.5 & 8.9 & 9.4 & 9.6 & 9.5 & 10.1 \\
\hline
\end{tabular}

Source: Afghan authorities; and Fund staff estimates and projections.

$1 /$ Excluding the narcotics economy.

$2 /$ It is assumed that all recurrent expenditure is moved onto the budget during 2014 and 2015 . The actual rate of transfer on-budget remains uncertain and subject to donor confidence in public financial managment mechanisms.

3/ Defined as domestic revenues minus operating expenditures.

$4 /$ In months of next year's imports of goods and services, excluding imports for reexport.

5/ Estimated direct expenditures by the international community on public projects not included in the government budget.

6/ After HIPC and MDRI debt relief, as well as debt relief beyond HIPC relief from Paris Club creditors. 
Table 3a. Islamic Republic of Afghanistan: Central Government Budget, 2010/11-2013
(In billions of Afghanis)

\begin{tabular}{|c|c|c|c|c|c|c|c|c|c|c|c|c|c|c|c|c|c|}
\hline & \multirow{3}{*}{$\begin{array}{r}\frac{2010 / 11}{} \\
\text { Solar Year } \\
\text { Act. }\end{array}$} & \multicolumn{6}{|c|}{$2011 / 12$} & \multirow{2}{*}{\multicolumn{2}{|c|}{$\begin{array}{l}2012 / 13 \\
\text { Solar Year } \\
\end{array}$}} & \multicolumn{6}{|c|}{2012} & \multirow{3}{*}{ 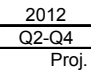 } & \multirow{3}{*}{$\frac{2013}{\frac{2013}{\text { Year }}}$} \\
\hline & & \multicolumn{2}{|c|}{ Q3 } & \multicolumn{2}{|c|}{ Q4 } & \multicolumn{2}{|c|}{ Solar Year } & & & \multicolumn{2}{|c|}{ Q2 } & \multicolumn{2}{|c|}{ Q3 } & \multicolumn{2}{|c|}{ Q4 } & & \\
\hline & & Prog. & Act. & Prog. & Est. & Prog. & Est. & \multicolumn{2}{|c|}{\begin{tabular}{l|}
$\frac{\text { Solar Year }}{2}$ \\
Prog.
\end{tabular}} & Prog. & Proj. & Prog. & Proj. & Prog. & Proj. & & \\
\hline Revenues and grants & 160.4 & 63.3 & 47.9 & 53.7 & 63.8 & 204.4 & 198.5 & 229.3 & 232.4 & 46.5 & 46.5 & 58.5 & 58.5 & 62.7 & 71.5 & 176.5 & 266.6 \\
\hline Domestic revenues & 80.4 & 24.9 & 23.1 & 27.2 & 28.1 & 98.8 & 97.3 & 109.0 & 113.5 & 23.8 & 23.8 & 26.6 & 26.6 & 27.8 & 34.7 & 85.1 & 129.0 \\
\hline Tax revenues & 66.4 & 23.4 & 20.0 & 25.4 & 18.4 & 82.1 & 71.8 & 93.0 & 88.6 & 20.3 & 20.3 & 22.7 & 22.7 & 23.7 & 23.4 & 66.5 & 100.4 \\
\hline Income, profits, and capital gains & 19.4 & 6.7 & 6.2 & 7.6 & 5.7 & 23.7 & 21.3 & 27.1 & 26.7 & 5.9 & 5.9 & 6.6 & 6.6 & 6.9 & 7.5 & 20.0 & 30.9 \\
\hline International trade and transactions & 27.7 & 9.4 & 7.6 & 9.4 & 7.5 & 33.8 & 30.1 & 37.6 & 35.6 & 8.2 & 8.2 & 9.2 & 9.2 & 9.6 & 9.3 & 26.7 & 38.4 \\
\hline Goods and services & 16.3 & 6.4 & 5.1 & 7.0 & 4.4 & 21.0 & 17.1 & 24.2 & 22.1 & 5.3 & 5.3 & 5.9 & 5.9 & 6.2 & 5.4 & 16.6 & 25.5 \\
\hline Other & 3.0 & 0.9 & 1.1 & 1.4 & 0.8 & 3.6 & 3.2 & 4.1 & 4.2 & 0.9 & 0.9 & 1.0 & 1.0 & 1.0 & 1.3 & 3.2 & 5.6 \\
\hline Nontax revenues & 14.0 & 1.5 & 3.1 & 1.9 & 9.6 & 16.7 & 25.6 & 16.0 & 24.8 & 3.5 & 3.5 & 3.9 & 3.9 & 4.1 & 11.2 & 18.6 & 28.7 \\
\hline Grants to operating budget & 54.5 & 20.9 & 15.6 & 13.7 & 23.5 & 65.9 & 70.3 & 75.0 & 76.1 & 15.2 & 15.2 & 18.8 & 18.8 & 20.5 & 25.1 & 59.1 & 92.4 \\
\hline Afghanistan Reconstruction Trust Fund (ARTF) & 15.4 & 5.9 & 0.1 & 2.3 & 4.8 & 9.4 & 6.0 & 12.1 & 11.4 & 2.6 & 2.6 & 5.2 & 5.2 & 0.0 & 0.7 & 8.5 & 15.5 \\
\hline Law and Order Trust Fund for Afghanistan (LOTFA) & 24.4 & 9.6 & 6.2 & 6.5 & 10.5 & 28.0 & 28.6 & 31.3 & 32.2 & 6.9 & 6.9 & 7.2 & 7.2 & 10.3 & 10.2 & 24.2 & 40.0 \\
\hline NATO Training Mission - Afghanistan (NTM-A) and other grants & 14.6 & 5.4 & 9.3 & 4.9 & 8.2 & 28.5 & 35.6 & 31.6 & 32.6 & 5.7 & 5.7 & 6.5 & 6.5 & 10.1 & 14.2 & 26.4 & 36.9 \\
\hline Grants to development budget & 25.6 & 17.5 & 9.2 & 12.8 & 12.3 & 39.6 & 30.8 & 45.3 & 42.8 & 7.5 & 7.5 & 13.1 & 13.1 & 14.4 & 11.7 & 32.3 & 45.2 \\
\hline Total expenditures & 154.0 & 63.5 & 54.0 & 65.9 & 73.8 & 204.8 & 203.7 & 244.4 & 246.2 & 38.1 & 38.1 & 65.5 & 65.5 & 61.1 & 76.6 & 180.1 & 275.2 \\
\hline Operating expenditures & 110.5 & 46.7 & 39.0 & 46.0 & 52.3 & 151.4 & 150.3 & 180.3 & 180.3 & 33.9 & 33.9 & 45.8 & 45.8 & 43.2 & 50.7 & 130.4 & 202.3 \\
\hline Wages and salaries & 86.5 & 27.8 & 28.5 & 37.9 & 35.3 & 113.7 & 111.8 & 133.0 & 136.5 & 24.2 & 24.2 & 33.8 & 33.8 & 31.7 & 45.4 & 103.4 & 149.9 \\
\hline Purchases of goods and services & 17.2 & 5.2 & 7.7 & 11.7 & 11.5 & 23.3 & 25.9 & 26.2 & 24.6 & 2.4 & 2.4 & 5.6 & 5.6 & 5.6 & 6.4 & 14.4 & 28.6 \\
\hline Transfers, subsidies, and other & 1.7 & 1.1 & 0.5 & 1.4 & 0.6 & 3.8 & 2.0 & 4.3 & 5.3 & 0.5 & 0.5 & 1.2 & 1.2 & 1.2 & 2.9 & 4.6 & 6.1 \\
\hline Pensions & 3.4 & 11.5 & 1.5 & -7.5 & 3.1 & 6.1 & 7.1 & 11.9 & 9.5 & 6.2 & 6.2 & 4.4 & 4.4 & 3.7 & -4.7 & 6.0 & 12.9 \\
\hline Capital expenditures & 1.6 & 0.6 & 0.6 & 2.3 & 1.6 & 3.4 & 2.7 & 3.8 & 3.4 & 0.2 & 0.2 & 0.6 & 0.6 & 0.7 & 0.6 & 1.4 & 4.0 \\
\hline Interest & 0.1 & 0.5 & 0.3 & 0.3 & 0.2 & 1.0 & 0.8 & 1.1 & 0.9 & 0.4 & 0.4 & 0.2 & 0.2 & 0.4 & 0.1 & 0.7 & 0.8 \\
\hline $\begin{array}{l}\text { Development expenditures } \\
\text { Of which: }\end{array}$ & 43.6 & 16.8 & 15.0 & 19.9 & 21.5 & 53.4 & 53.4 & 64.1 & 65.9 & 4.2 & 4.2 & 19.7 & 19.7 & 17.8 & 25.8 & 49.7 & 72.9 \\
\hline Infrastructure and natural resources & 20.7 & 9.1 & 5.6 & 10.4 & 10.2 & 25.5 & 21.8 & 30.6 & 26.9 & 1.7 & 1.7 & 8.6 & 8.6 & 9.5 & 13.3 & 23.6 & 34.7 \\
\hline Education & 5.2 & 3.1 & 1.5 & 1.4 & 2.8 & 6.4 & 6.2 & 7.6 & 7.6 & 0.6 & 0.6 & 3.4 & 3.4 & 2.5 & 1.9 & 5.9 & 8.7 \\
\hline Health & 4.3 & 1.5 & 1.2 & 2.0 & 1.9 & 5.3 & 4.9 & 6.3 & 6.0 & 0.5 & 0.5 & 1.8 & 1.8 & 1.7 & 2.6 & 4.9 & 7.2 \\
\hline Operating balance excluding grants & -30.1 & -21.8 & -15.9 & -18.8 & -24.2 & -52.6 & -53.0 & -71.3 & -66.8 & -10.1 & -10.1 & -19.2 & -19.2 & -15.4 & -16.1 & -45.3 & -73.3 \\
\hline Overall budget balance including grants & 6.4 & -0.2 & -6.1 & -12.1 & -10.0 & -0.4 & -5.3 & -15.1 & -13.7 & 8.5 & 8.5 & -6.9 & -6.9 & 1.6 & -5.1 & -3.6 & -8.6 \\
\hline Central bank compensation for Kabul Bank costs & $\ldots$ & 0.0 & 0.0 & -1.8 & 0.0 & -34.1 & -37.6 & 0.0 & 0.0 & 0.0 & 0.0 & 0.0 & 0.0 & 0.0 & 0.0 & 0.0 & 0.0 \\
\hline Float and discrepancy ("+" = recorded expenditures not yet executed) & 2.7 & 0.8 & 4.8 & & 9.6 & $\ldots$ & 12.7 & & & & $\ldots$ & $\ldots$ & $\ldots$ & $\ldots$ & & $\ldots$ & \\
\hline Financing & -9.1 & -0.6 & 1.3 & 12.1 & 0.3 & 34.5 & 30.2 & 15.1 & 13.7 & -8.5 & -8.5 & 7.1 & 6.9 & -1.6 & 5.1 & 3.6 & 8.6 \\
\hline Sale of nonfinanci & 2.5 & 0.0 & 0.0 & 0.0 & 1.2 & 2.3 & 1. & 5.4 & 2. & 0.0 & 2.5 & 0.1 & 0.0 & 0.0 & 0.0 & 2.5 & 3.1 \\
\hline External loans (ne & 2.8 & 3.3 & 0.3 & 3.3 & 0.6 & 7.4 & 1.6 & 9.3 & 2.1 & 0.1 & 0.2 & 0.8 & 1.4 & 4.2 & 0.7 & 2.3 & 5.5 \\
\hline Domestic (net) & -14.4 & -3.9 & 1.0 & 8.8 & -1.5 & 24.8 & 26.7 & 0.4 & 9.2 & -8.6 & -11.2 & 6.2 & 5.6 & -5.8 & 4.4 & -1.2 & 0.0 \\
\hline Central bank & -14.4 & -3.9 & 1.0 & 8.8 & -1.5 & 24.8 & 26.7 & 0.4 & 9.2 & -8.6 & -11.2 & 6.2 & 5.6 & -5.8 & 4.4 & -1.2 & 0.0 \\
\hline Memora & & & & & & & & & & & & & & & & & \\
\hline Domestic revenues as & 72.8 & 53.3 & 59.3 & 59.2 & 53.7 & 65.3 & 64.7 & 60.4 & 62.9 & 70.3 & 70.3 & 58.1 & 58.1 & 64.3 & 68.3 & 65.3 & 63.8 \\
\hline Central bank capita & $\ldots$ & 33.9 & 34.1 & 31.5 & 34.8 & 31.5 & 34.8 & 25.7 & 27.1 & 33.7 & 33.7 & 29.4 & 29.4 & 28.3 & 28.3 & 28.3 & 23.2 \\
\hline Indicative & & $\ldots$ & $\ldots$ & $\ldots$ & $\ldots$ & 18.0 & & 19.4 & & $\ldots$ & $\ldots$ & & $\ldots$ & $\ldots$ & & & \\
\hline nces 3 & 27.9 & $\ldots$ & $\ldots$ & $\ldots$ & $\ldots$ & 20.4 & 34.5 & 5.6 & 15.8 & $\ldots$ & $\ldots$ & $\ldots$ & ... & ... & … & 28.2 & 32.8 \\
\hline External budget $4 /$ & 91.5 & $\cdots$ & $\cdots$ & $\cdots$ & $\cdots$ & 190.7 & 100.2 & 190.8 & 127.9 & ... & $\cdots$ & $\cdots$ & $\begin{array}{c}\cdots \\
\cdots\end{array}$ & $\cdots$ & $\cdots$ & 96.2 & 138.9 \\
\hline
\end{tabular}

Source: Afghan authorities; and Fund staff estimates and projection

1/ Includes signature bonus payments for the Aynak copper mine.

2/ Propoor spending covers the ministry of education, the ministry of labor and social affairs, martyrs and disabled, and the ministry of public health.

4/ Estimated direct expenditures by donors on public projects not included in the central government budget. 

Table 3b. Islamic Republic of Afghanistan: Central Government Budget, 2010/11-2013

\begin{tabular}{|c|c|c|c|c|c|c|c|c|c|c|c|c|c|c|c|c|c|}
\hline & \multirow{3}{*}{ 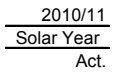 } & \multicolumn{6}{|c|}{$2011 / 12$} & \multirow{2}{*}{\multicolumn{2}{|c|}{$\begin{array}{l}2012 / 13 \\
\text { Solar Year } \\
\end{array}$}} & \multicolumn{6}{|c|}{2012} & \multicolumn{2}{|l|}{2012} \\
\hline & & $\mathrm{Q3}_{3}$ & & $\mathrm{Q}^{2}$ & & Solar Y & & & & $Q$ & & & & Q4 & & Q2-Q4 & Year \\
\hline & & Prog. & Act. & Prog. & Est. & Prog. & Est. & Prog. & Proj. & Prog. & Proj. & Prog. & Proj. & Prog. & Proj. & Proj. & Proj. \\
\hline Revenues and grants & 22.0 & 7.2 & 5.5 & 6.1 & 7.3 & 23.4 & 22.7 & 23.7 & 22.6 & 6.0 & 6.0 & 7.6 & 7.6 & 8.1 & 9.3 & 22.9 & 23.4 \\
\hline Domestic revenues & 11.0 & 2.8 & 2.6 & 3.1 & 3.2 & 11.3 & 11.1 & 11.3 & 11.0 & 3.1 & 3.1 & 3.5 & 3.5 & 3.6 & 4.5 & 11.0 & 11.3 \\
\hline Tax revenues & 9.1 & 2.7 & 2.3 & 2.9 & 2.1 & 9.4 & 8.2 & 9.6 & 8.6 & 2.6 & 2.6 & 2.9 & 2.9 & 3.1 & 3.0 & 8.6 & 8.8 \\
\hline ofits, and capital gains & 2.7 & 0.8 & 0.7 & 0.9 & 0.7 & 2.7 & 2.4 & 2.8 & 2.6 & 0.8 & 0.8 & 0.9 & 0.9 & 0.9 & 1.0 & 2.6 & 2.7 \\
\hline International trade and transactions & 3.8 & 1.1 & 0.9 & 1.1 & 0.9 & 3.9 & 3.4 & 3.9 & 3.5 & 1.1 & 1.1 & 1.2 & 1.2 & 1.2 & 1.2 & 3.5 & 3.4 \\
\hline Goods and services & 2.2 & 0.7 & 0.6 & 0.8 & 0.5 & 2.4 & 2.0 & 2.5 & 2.1 & 0.7 & 0.7 & 0.8 & 0.8 & 0.8 & 0.7 & 2.1 & 2.2 \\
\hline Other & 0.4 & 0.1 & 0.1 & 0.2 & 0.1 & 0.4 & 0.4 & 0.4 & 0.4 & 0.1 & 0.1 & 0.1 & 0.1 & 0.1 & 0.2 & 0.4 & 0.5 \\
\hline Nontax revenues & 1.9 & 0.2 & 0.4 & 0.2 & 1.1 & 1.9 & 2.9 & 1.7 & 2.4 & 0.5 & 0.5 & 0.5 & 0.5 & 0.5 & 1.5 & 2.4 & 2.5 \\
\hline $\begin{array}{l}\text { Grants to operating budget } \\
\text { Afghanistan Reconstruction Trust Fund }\end{array}$ & $\begin{array}{l}7.5 \\
2.1\end{array}$ & $\begin{array}{l}2.4 \\
0.7\end{array}$ & $\begin{array}{l}1.8 \\
0.0\end{array}$ & $\begin{array}{l}1.6 \\
0.3\end{array}$ & 2.7 & $\begin{array}{l}7.5 \\
1.1\end{array}$ & $\begin{array}{l}8.0 \\
0.7\end{array}$ & $\begin{array}{l}7.7 \\
1.2\end{array}$ & $\begin{array}{l}7.4 \\
1.1\end{array}$ & 2.0 & 2.0 & 2.4 & 2.4 & 2.7 & 3.3 & 7.7 & $\begin{array}{l}8.1 \\
1.4\end{array}$ \\
\hline Law and Order Trust Fund for Afghanistan (LOTFA) & 3.3 & 1.1 & 0.7 & 0.7 & 1.2 & 3.2 & 3.3 & 3.2 & 3.1 & 0.9 & 0.9 & 0.9 & 0.9 & 1.3 & 1.3 & $\begin{array}{l}1.1 \\
3.1\end{array}$ & $\begin{array}{l}1.4 \\
3.5\end{array}$ \\
\hline NATO Training Mission - Afghanistan (NTM-A) and other grants & 2.0 & 0.6 & 1.1 & 0.6 & 0.9 & 3.3 & 4.1 & 3.3 & 3.2 & 0.7 & 0.7 & 0.8 & 0.8 & 1.3 & 1.8 & 3.4 & 3.2 \\
\hline Grants to development budget & 3.5 & 2.0 & 1.0 & 1.5 & 1.4 & 4.5 & 3.5 & 4.7 & 4.2 & 1.0 & 1.0 & 1.7 & 1.7 & 1.9 & 1.5 & 4.2 & 4.0 \\
\hline Total expenditures & 21.1 & 7.3 & 6.2 & 7.5 & 8.4 & 23.4 & 23.3 & 25.2 & 23.9 & 4.9 & 4.9 & 8.5 & 8.5 & 7.9 & 9.9 & 23.4 & 24.1 \\
\hline Operating expenditures & 15.1 & 5.3 & 4.5 & 5.3 & 6.0 & 17.3 & 17.2 & 18.6 & 17.5 & 4.4 & 4.4 & 5.9 & 5.9 & 5.6 & 6.6 & 16.9 & 17.7 \\
\hline Wages and salaries & 11.8 & 3.2 & 3.3 & 4.3 & 4.0 & 13.0 & 12.8 & 13.7 & 13.3 & 3.1 & 3.1 & 4.4 & 4.4 & 4.1 & 5.9 & 13.4 & 13.2 \\
\hline oods and s & 2.4 & 0.6 & 0.9 & 1.3 & 1.3 & 2.7 & 3.0 & 2.7 & 2.4 & 0.3 & 0.3 & 0.7 & 0.7 & 0.7 & 0.8 & 1.9 & 2.5 \\
\hline Transfers, subsidies, and other & 0.2 & 0.1 & 0.1 & 0.2 & 0.1 & 0.4 & 0.2 & 0.4 & 0.5 & 0.1 & 0.1 & 0.2 & 0.2 & 0.2 & 0.4 & 0.6 & 0.5 \\
\hline Pensions & 0.5 & 1.3 & 0.2 & -0.9 & 0.4 & 0.7 & 0.8 & 1.2 & 0.9 & 0.8 & 0.8 & 0.6 & 0.6 & 0.5 & -0.6 & 0.8 & 1.1 \\
\hline Capital expenditures & 0.2 & 0.1 & 0.1 & 0.3 & 0.2 & 0.4 & 0.3 & 0.4 & 0.3 & 0.0 & 0.0 & 0.1 & 0.1 & 0.1 & 0.1 & 0.2 & 0.3 \\
\hline Interest & 0.0 & 0.1 & 0.0 & 0.0 & 0.0 & 0.1 & 0.1 & 0.1 & 0.1 & 0.1 & 0.1 & 0.0 & 0.0 & 0.0 & 0.0 & 0.1 & 0.1 \\
\hline $\begin{array}{l}\text { Development expenditures } \\
\text { Of which: }\end{array}$ & 6.0 & 1.9 & 1.7 & 2.3 & 2.5 & 6.1 & 6.1 & 6.6 & 6.4 & 0.5 & 0.5 & 2.6 & 2.6 & 2.3 & 3.3 & 6.4 & 6.4 \\
\hline Infrastructure and natural resources & 2.8 & 1.0 & 0.6 & 1.2 & 1.2 & 2.9 & 2.5 & 3.2 & 2.6 & 0.2 & 0.2 & 1.1 & 1.1 & 1.2 & 1.7 & 3.1 & 3.0 \\
\hline Education & 0.7 & 0.4 & 0.2 & 0.2 & 0.3 & 0.7 & 0.7 & 0.8 & 0.7 & 0.1 & 0.1 & 0.4 & 0.4 & 0.3 & 0.2 & 0.8 & 0.8 \\
\hline Health & 0.6 & 0.2 & 0.1 & 0.2 & 0.2 & 0.6 & 0.6 & 0.7 & 0.6 & 0.1 & 0.1 & 0.2 & 0.2 & 0.2 & 0.3 & 0.6 & 0.6 \\
\hline Operating balance excluding grants & -4.1 & -2.5 & -1.8 & -2.1 & -2.8 & -6.0 & -6.1 & -7.4 & -6.5 & -1.3 & -1.3 & -2.5 & -2.5 & -2.0 & -2.1 & -5.9 & -6.4 \\
\hline Overall budget balance including grants & 0.9 & 0.0 & -0.7 & -1.4 & -1.1 & 0.0 & -0.6 & -1.6 & -1.3 & 1.1 & 1.1 & -0.9 & -0.9 & 0.2 & -0.7 & -0.5 & -0.8 \\
\hline Central bank compensation for Kabul Bank costs & $\ldots$ & 0.0 & 0.0 & -0.2 & 0.0 & -3.9 & -4.3 & 0.0 & 0.0 & 0.0 & 0.0 & 0.0 & 0.0 & 0.0 & 0.0 & 0.0 & 0.0 \\
\hline Float and discrepancy ("+" = recorded expenditures not yet executed) & 0.4 & 0.1 & 0.6 & $\ldots$ & 1.1 & $\cdots$ & $\ldots$ & $\cdots$ & $\ldots$ & $\ldots$ & $\ldots$ & $\ldots$ & $\ldots$ & $\ldots$ & $\ldots$ & $\ldots$ & \\
\hline Financing & -1.2 & -0.1 & 0.1 & 1.4 & 0.0 & 3.9 & 3.5 & 1.6 & 1.3 & -1.1 & -1.1 & 0.9 & 0.9 & -0.2 & 0.7 & 0.5 & 0.5 \\
\hline onfinancial assets $1 /$ & 0.3 & 0.0 & 0.0 & 0.0 & 0.1 & 0.3 & 0.2 & 0.6 & 0.2 & 0.0 & 0.3 & 0.0 & 0.0 & 0.0 & 0.0 & 0.3 & 0.0 \\
\hline External loans (net) & 0.4 & 0.4 & 0.0 & 0.4 & 0.1 & 0.8 & 0.2 & 1.0 & 0.2 & 0.0 & 0.0 & 0.1 & 0.2 & 0.5 & 0.1 & 0.3 & 0.5 \\
\hline Domestic (net) & -2.0 & -0.4 & 0.1 & 1.0 & -0.2 & 2.8 & 3.1 & 0.0 & 0.9 & -1.1 & -1.5 & 0.8 & 0.7 & -0.8 & 0.6 & -0.2 & 0.0 \\
\hline Central bank & -2.0 & -0.4 & 0.1 & 1.0 & -0.2 & 2.8 & 3.1 & 0.0 & 0.9 & -1.1 & -1.5 & 0.8 & 0.7 & -0.8 & 0.6 & -0.2 & 0.0 \\
\hline Memor & & & & & & & & & & & & & & & & & \\
\hline ating expenditures & 73 & 53 & 59 & 59 & 54 & 65.3 & 64.7 & 60.4 & 62.9 & 70.3 & 70.3 & $\begin{array}{r}58.1 \\
38\end{array}$ & 58.1 & 64.3 & 68.3 & 65.3 & 63.8 \\
\hline id (end-of-period stock) & $\cdots$ & 3.9 & ( & 3.6 & 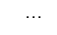 & 3.6 & 4.0 & 2.7 & 2.6 & 4.4 & 4.4 & 3.8 & 3.8 & 3.7 & 3.7 & 3.7 & 2.0 \\
\hline propoor spending $2 /$ & & & $\cdots$ & $\cdots$ & $\ldots$ & 2.1 & & 2.0 & & ... & $\cdots$ & $\ldots$ & $\cdots$ & $\ldots$ & .. & & \\
\hline Discretionary cash balances $3 /$ & 3.8 & $\cdots$ & $\cdots$ & $\cdots$ & $\cdots$ & 2.3 & 3.9 & 0.6 & 1.5 & ... & $\cdots$ & ... & $\cdots$ & ... & $\cdots$ & 3.7 & 2.9 \\
\hline External budget 4/ & 12.5 & & & & & 21.8 & 11.5 & 19.7 & 12.4 & & & & & & & 12.5 & 12.2 \\
\hline Nominal GDP (in billions of Afghanis) & 730 & 875 & 875 & 875 & 875 & 874.7 & 874.7 & 968.0 & 1028.1 & 771.1 & 771.1 & 771.1 & 771.1 & 771.1 & 771.1 & 771.1 & 1140.1 \\
\hline
\end{tabular}

Source: Afghan authorities; and Fund staff estimates and projections.

1/ Includes signature bonus payments for the Aynak copper mine.
2/ Propoor spending covers the ministry of educcition, the ministry of labor and social affairs, martyrs and disabled, and the ministry of public health.
$3 /$ Government deposits at the central bank exclude earmarked grants

4/ Estimated direct expenditures by donors on public projects not included in the central government budget. 
Table 4. Islamic Republic of Afghanistan: Total On-Budget and Off-Budget Fiscal Financing Needs, 2012-25 (In percent of GDP)

\begin{tabular}{|c|c|c|c|c|c|c|c|c|c|c|c|c|c|c|}
\hline & 2012 (Q2-Q4) & 2013 & 2014 & 2015 & 2016 & 2017 & 2018 & 2019 & 2020 & 2021 & 2022 & 2023 & 2024 & $\begin{array}{r}2025 \\
\text { Proi }\end{array}$ \\
\hline & Proj & Proj & Proj & Proj & Proj & Proj & Proj & Proj & Proj & Proj & Proj & Proj & Proj & Proj \\
\hline Revenue and grants & 57.8 & 54.9 & 13.4 & 14.0 & 14.5 & 14.5 & 15.6 & 16.1 & 16.2 & 16.5 & 16.7 & 16.7 & 16.9 & 17.0 \\
\hline Domestic revenues & 11.0 & 11.3 & 13.4 & 14.0 & 14.5 & 14.5 & 15.6 & 16.1 & 16.2 & 16.5 & 16.7 & 16.7 & 16.9 & 17.0 \\
\hline Tax revenues & 8.6 & 8.7 & 10.5 & 10.9 & 11.0 & 10.8 & 11.6 & 11.7 & 11.7 & 11.8 & 12.0 & 12.1 & 12.2 & 12.3 \\
\hline Non-tax revenues & 2.5 & 2.6 & 2.5 & 2.5 & 2.5 & 2.6 & 2.6 & 2.6 & 2.5 & 2.6 & 2.6 & 2.6 & 2.6 & 2.6 \\
\hline Extractive industries 1/ & 0.0 & 0.0 & 0.4 & 0.6 & 1.0 & 1.2 & 1.4 & 1.8 & 1.9 & 2.1 & 2.1 & 2.1 & 2.1 & 2.1 \\
\hline Grants & 46.7 & 43.6 & $\ldots$ & $\ldots$ & $\ldots$ & $\ldots$ & $\ldots$ & $\ldots$ & $\ldots$ & $\ldots$ & $\ldots$ & $\ldots$ & $\ldots$ & $\ldots$ \\
\hline Expenditures (incl. off-budget spending) & 58.2 & 55.6 & 51.9 & 48.5 & 43.0 & 40.2 & 39.6 & 38.6 & 38.5 & 38.6 & 38.9 & 38.9 & 38.9 & 39.0 \\
\hline Security 21 & 32.4 & 29.5 & 27.0 & 24.8 & 19.7 & 16.3 & 16.1 & 15.7 & 15.4 & 15.2 & 15.0 & 14.8 & 14.7 & 14.5 \\
\hline Wages & 9.2 & 9.1 & 8.7 & 8.2 & 6.5 & 5.2 & 5.0 & 4.8 & 4.6 & 4.4 & 4.3 & 4.2 & 4.0 & 3.9 \\
\hline Goods and services, capital expenditures, operations and maintanance & 23.1 & 20.5 & 18.3 & 16.6 & 13.3 & 11.1 & 11.0 & 10.9 & 10.8 & 10.8 & 10.7 & 10.7 & 10.6 & 10.6 \\
\hline Nonsecurity operating & 12.3 & 12.9 & 11.8 & 12.1 & 12.2 & 12.4 & 12.7 & 13.0 & 13.3 & 13.6 & 14.0 & 14.1 & 14.3 & 14.5 \\
\hline Wages & 4.2 & 4.1 & 4.1 & 4.2 & 4.2 & 4.2 & 4.4 & 4.5 & 4.6 & 4.8 & 4.9 & 4.9 & 4.9 & 4.9 \\
\hline Of which: Capacity Building for Results and Externally Funded Staff & 0.0 & 0.1 & 0.2 & 0.2 & 0.2 & 0.2 & 0.4 & 0.6 & 0.7 & 0.9 & 1.0 & 1.0 & 1.0 & 1.0 \\
\hline Goods and services, capital expenditures, operations and maintanance & 6.7 & 7.1 & 6.0 & 6.3 & 6.5 & 6.6 & 6.7 & 6.9 & 7.1 & 7.2 & 7.4 & 7.6 & 7.8 & 7.9 \\
\hline Transfers, subsides, pensions & 1.4 & 1.7 & 1.6 & 1.5 & 1.5 & 1.5 & 1.5 & 1.5 & 1.5 & 1.5 & 1.5 & 1.5 & 1.5 & 1.5 \\
\hline Interest & 0.1 & 0.1 & 0.1 & 0.1 & 0.1 & 0.1 & 0.1 & 0.1 & 0.1 & 0.1 & 0.1 & 0.1 & 0.1 & 0.1 \\
\hline Development & 13.6 & 13.1 & 13.2 & 11.7 & 11.1 & 11.5 & 10.8 & 9.8 & 9.8 & 9.8 & 10.0 & 9.9 & 10.0 & 10.0 \\
\hline On-budget & 6.4 & 6.4 & 6.6 & 6.7 & 6.9 & 7.1 & 7.2 & 7.4 & 7.6 & 7.8 & 8.0 & 8.0 & 8.0 & 8.0 \\
\hline Off-budget $3 /$ & 7.1 & 6.8 & 6.6 & 4.9 & 4.2 & 4.4 & 3.6 & 2.4 & 2.2 & 2.0 & 2.0 & 2.0 & 2.0 & 2.0 \\
\hline Overall budget balance (excl. grants) & -12.3 & -12.8 & -11.8 & -27.8 & -24.3 & -21.2 & -20.5 & -20.1 & -20.1 & -20.2 & -20.3 & -20.2 & -20.0 & -20.0 \\
\hline Overall budget balance (incl. grants) & -0.5 & -0.8 & -11.8 & -27.8 & -24.3 & -21.2 & -20.5 & -20.1 & -20.1 & -20.2 & -20.3 & -20.2 & -20.0 & -20.0 \\
\hline Overall balance incl. off-budget spending (incl. grants) $4 /$ & -0.5 & -0.8 & -39.8 & -34.5 & -28.5 & -25.6 & -24.0 & -22.5 & -22.3 & -22.2 & -22.3 & -22.2 & -22.0 & -22.0 \\
\hline Identified financing & 0.5 & 0.8 & 1.0 & 0.9 & 1.5 & 1.0 & 0.7 & 0.6 & 0.5 & 0.7 & 0.5 & 0.7 & 0.8 & 1.0 \\
\hline Sale of Nonfinancial Assets & 0.3 & 0.3 & 0.0 & 0.4 & 2.5 & 0.4 & 0.3 & 0.0 & 0.0 & 0.0 & 0.0 & 0.0 & 0.0 & 0.0 \\
\hline External Loans & 0.3 & 0.5 & 0.5 & 0.5 & 0.5 & 0.5 & 0.4 & 0.5 & 0.5 & 0.5 & 0.5 & 0.5 & 0.5 & 0.5 \\
\hline Net domestic financing & -0.2 & 0.0 & 0.6 & 0.0 & -1.5 & 0.2 & 0.0 & 0.2 & 0.1 & 0.2 & 0.1 & 0.2 & 0.3 & 0.5 \\
\hline Of which: Amortization payments & -0.4 & -0.3 & -0.1 & -0.1 & -0.1 & 0.0 & -0.2 & -0.2 & -0.2 & -0.2 & -0.2 & 0.0 & 0.0 & 0.0 \\
\hline Financing gap & 0.0 & 0.0 & 38.8 & 33.6 & 27.1 & 24.6 & 23.3 & 21.8 & 21.8 & 21.5 & 21.7 & 21.4 & 21.2 & 21.0 \\
\hline Security & $\ldots$ & $\ldots$ & 23.5 & 20.9 & 15.8 & 12.3 & 12.0 & 11.6 & 11.2 & 10.9 & 10.6 & 10.3 & 10.1 & 9.8 \\
\hline Development and budget support & $\ldots$ & $\ldots$ & 15.3 & 12.7 & 11.3 & 12.3 & 11.3 & 10.2 & 10.6 & 10.6 & 11.1 & 11.1 & 11.2 & 11.2 \\
\hline \multicolumn{15}{|l|}{ Memorandum items } \\
\hline Total revenue from extractive industries incl. signature bonuses & 0.3 & 0.3 & 0.4 & 1.0 & 3.5 & 1.5 & 1.7 & 1.8 & 1.9 & 2.1 & 2.1 & 2.1 & 2.1 & 2.1 \\
\hline Security spending paid for by Government of Afghanistan & 3.4 & 3.5 & 3.5 & 3.9 & 3.9 & 4.0 & 4.1 & 4.1 & 4.2 & 4.3 & 4.4 & 4.5 & 4.6 & 4.7 \\
\hline Development spending paid for by Government of Afghanistan & 2.3 & 2.4 & 2.5 & 3.4 & 3.4 & 2.6 & 2.9 & 3.0 & 2.4 & 2.3 & 2.0 & 2.0 & 2.0 & 2.0 \\
\hline Non-security operations and maintanance cost & 5.3 & 5.4 & 4.2 & 4.5 & 4.5 & 4.6 & 4.7 & 4.7 & 4.8 & 4.9 & 5.0 & 5.1 & 5.2 & 5.3 \\
\hline Of which: Off-budget & 5.3 & 5.4 & 2.9 & 1.8 & 0.0 & 0.0 & 0.0 & 0.0 & 0.0 & 0.0 & 0.0 & 0.0 & 0.0 & 0.0 \\
\hline Net Public Debt & 8.7 & 6.6 & 6.9 & 7.1 & 7.8 & 7.8 & 8.3 & 8.5 & 8.5 & 8.9 & 9.4 & 9.6 & 9.5 & 10.1 \\
\hline Of which: Domestic debt & $\ldots$ & $\ldots$ & 0.2 & 0.4 & 1.1 & 1.0 & 1.6 & 1.7 & 1.7 & 2.0 & 2.4 & 2.5 & 2.2 & 2.6 \\
\hline
\end{tabular}

Source: Afghan authorities; International Security Assistance Force; and Fund staff estimates and projections.

$1 /$ Excludes proceeds from the signature bonuses from natural resource projects, as recorded in the sale of nonfinancial assets.

2/ ISAF estimates ANSF costs of USD 4.1bn in 2017. This cost is projected both backwards to 2012 and forwards to 2025. The remainder includes additional security sector costs, e.g., wages outside of the MoD and Mol in MoFA, PPS, GDNS.

4/ For 2014, augmented by expenses related to central bank capitalization not included above the line. 
Table 5a. Islamic Republic of Afghanistan: Central Bank Balance Sheet, 2010-2013 (at market exchange rates)

\begin{tabular}{|c|c|c|c|c|c|c|c|c|c|c|c|c|c|}
\hline & \multirow{2}{*}{$\begin{array}{r}2010 \\
\text { Mar. } 20 \\
\text { Act. }\end{array}$} & \multicolumn{4}{|c|}{2011} & \multicolumn{4}{|c|}{2012} & \multicolumn{4}{|c|}{2013} \\
\hline & & $\begin{array}{r}\text { Mar. } 20 \\
\text { Act. }\end{array}$ & $\begin{array}{r}\text { June } 21 \\
\text { Act. }\end{array}$ & $\begin{array}{r}\text { Sep. } 22 \\
\text { Act. }\end{array}$ & $\begin{array}{r}\text { Dec. } 21 \\
\text { Act. }\end{array}$ & $\begin{array}{r}\text { Mar. } 19 \\
\text { Act. }\end{array}$ & $\begin{array}{r}\text { June } 20 \\
\text { Proj. }\end{array}$ & $\begin{array}{r}\text { Sep. } 21 \\
\text { Proj. }\end{array}$ & $\begin{array}{r}\text { Dec. } 20 \\
\text { Proj. }\end{array}$ & $\begin{array}{r}\text { Mar } 20 \\
\text { Proj. }\end{array}$ & $\begin{array}{r}\text { June } 21 \\
\text { Proj. }\end{array}$ & $\begin{array}{r}\text { Sep } 22 \\
\text { Proj. }\end{array}$ & $\begin{array}{r}\text { Dec 21 } \\
\text { Proj. }\end{array}$ \\
\hline & \multicolumn{13}{|c|}{ (In billions of Afghanis, unless otherwise indicated) } \\
\hline Net foreign assets & 192.8 & 235.5 & 267.6 & 285.4 & 296.2 & 301.0 & 317.7 & 327.1 & 335.6 & 345.4 & 355.3 & 365.3 & 375.3 \\
\hline Foreign assets & 209.9 & 252.1 & 284.9 & 302.8 & 314.6 & 319.5 & 337.2 & 346.7 & 356.2 & 366.9 & 376.8 & 387.7 & 397.8 \\
\hline Foreign exchange reserves $1 /$ & 204.0 & 245.2 & 278.3 & 293.3 & 305.1 & 308.0 & 324.7 & 333.7 & 342.8 & 353.0 & 362.5 & 372.8 & 382.5 \\
\hline Other foreign assets & 5.9 & 7.0 & 6.6 & 9.5 & 9.5 & 11.5 & 12.6 & 13.0 & 13.4 & 13.9 & 14.3 & 14.8 & 15.3 \\
\hline Foreign liabilities & -17.1 & -16.6 & -17.3 & -17.4 & -18.4 & -18.5 & -19.6 & -19.6 & -20.6 & -21.5 & -21.5 & -22.4 & -22.4 \\
\hline Net domestic assets & -68.4 & -84.5 & -101.1 & -117.1 & -126.0 & -125.7 & -142.4 & -142.0 & -133.7 & -143.5 & -144.4 & -139.1 & -141.1 \\
\hline Domestic assets & -50.6 & -71.7 & -49.1 & -57.4 & -64.5 & -69.4 & -73.6 & -71.6 & -61.4 & -69.3 & -68.3 & -60.9 & -60.8 \\
\hline Net claims on general government & -34.7 & -46.9 & -19.4 & -24.0 & -24.0 & -25.1 & -38.3 & -34.2 & -31.3 & -34.0 & -33.2 & -34.7 & -34.8 \\
\hline Gross claims on government & 17.1 & 16.6 & 54.9 & 53.9 & 52.5 & 53.3 & 53.3 & 49.0 & 48.8 & 48.6 & 47.3 & 46.8 & 45.6 \\
\hline Of which: MoF promissory note $2 /$ & $\ldots$ & $\ldots$ & 37.6 & 36.6 & 34.1 & 34.8 & 33.7 & 29.4 & 28.3 & 27.1 & 25.8 & 24.5 & 23.2 \\
\hline Domestic currency deposits & -10.6 & -9.2 & -4.0 & -7.1 & -8.7 & -12.9 & -14.7 & -13.4 & -12.9 & -13.2 & -12.8 & -12.9 & -12.6 \\
\hline Foreign currency deposits $1 /$ & -41.2 & -54.4 & -70.3 & -70.8 & -67.8 & -65.4 & -76.9 & -69.8 & -67.3 & -69.4 & -67.7 & -68.7 & -67.7 \\
\hline Other claims & -15.9 & -24.8 & -29.7 & -33.4 & -40.5 & -44.3 & -35.3 & -37.3 & -30.1 & -35.3 & -35.1 & -26.2 & -26.0 \\
\hline Of which: Capital notes & -10.9 & -16.1 & -20.5 & -23.9 & -26.0 & -30.9 & -22.5 & -24.5 & -17.2 & -22.3 & -22.1 & -13.0 & -12.7 \\
\hline Other items net $3 /$ & -17.8 & -12.8 & -52.0 & -59.6 & -61.5 & -56.3 & -68.8 & -70.4 & -72.2 & -74.2 & -76.1 & -78.2 & -80.3 \\
\hline Reserve money & 124.4 & 151.0 & 166.4 & 168.3 & 170.1 & 175.3 & 175.3 & 185.1 & 202.0 & 202.0 & 210.9 & 226.2 & 234.3 \\
\hline Currency in circulation & 98.7 & 132.4 & 133.7 & 144.4 & 148.4 & 148.2 & 152.8 & 166.8 & 176.1 & 179.6 & 190.0 & 197.0 & 204.0 \\
\hline Bank deposits (Afghani denominated) & 25.7 & 18.5 & 32.7 & 24.0 & 21.8 & 27.1 & 22.5 & 18.3 & 25.8 & 22.4 & 20.8 & 29.2 & 30.3 \\
\hline \multicolumn{14}{|l|}{ Memorandum items: } \\
\hline \multicolumn{14}{|l|}{ Money growth (year-on-year in percent) } \\
\hline Reserve money & 17.1 & 21.3 & 34.5 & 24.1 & 21.9 & 16.1 & 5.3 & 9.9 & 18.7 & 15.2 & 20.3 & 22.2 & 16.0 \\
\hline Currency in circulation & 28.5 & 34.2 & 30.8 & 22.0 & 20.8 & 11.9 & 14.3 & 15.6 & 18.7 & 21.2 & 24.3 & 18.1 & 15.8 \\
\hline \multicolumn{14}{|l|}{ Gross international reserves } \\
\hline (In millions of U.S. dollars) & 4,209 & 5,403 & 5,946 & 6,069 & 6,208 & 6,234 & 6,458 & 6,590 & 6,721 & 6,871 & 7,005 & 7,153 & 7,286 \\
\hline Interest rate, 28-day capital notes (percent) & 4.3 & 2.4 & 2.3 & 2.2 & 2.1 & 2.0 & $\ldots$ & $\ldots$ & $\ldots$ & $\ldots$ & $\ldots$ & $\ldots$ & $\cdots$ \\
\hline
\end{tabular}

Source: Afghan authorities; and Fund staff estimates and projections.

1/ Includes Afghanistan's SDR holdings.

2/ Represents a claim on the ministry of finance equal to the outstanding balance on a lender of last resort loan, as specified by a promissory note issued by the ministry.

3/ Before May 2011, includes lender of last resort assistance to a now-bankrupt bank—extended in September 2011 (Af 18.1 billion) and April 2012 (Af 19.5 billion)—subsequently assumed by the ministry of finance through issuance of a promissory note (see footnote above). 
Table 5b. Islamic Republic of Afghanistan: Central Bank Balance Sheet, 2010-2013

(at program exchange rates) $1 /$

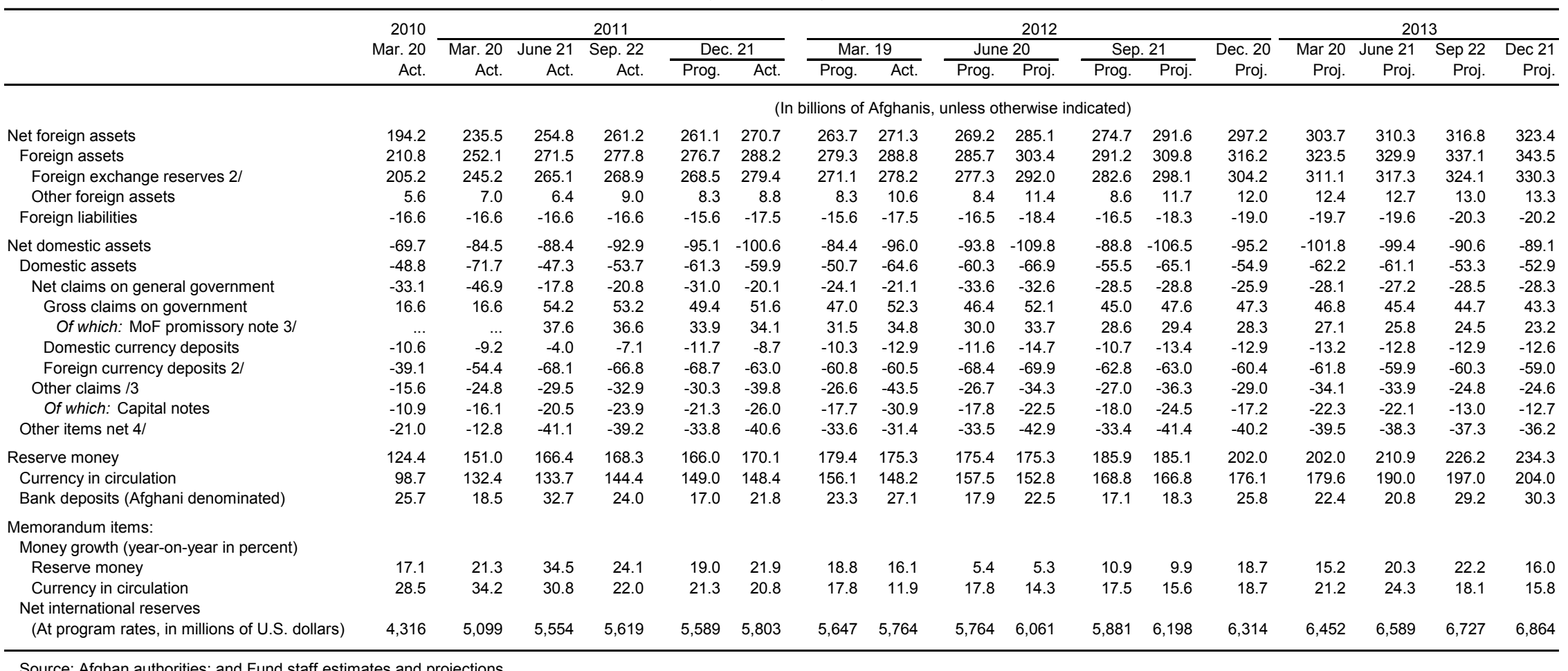

Source: Afghan authorities; and Fund staff estimates and projections.

1/ Program exchange rates as of March 20, 2011 applied to evaluate foreign currency-denominated components. The Afghani per U.S. dollar rate for that day was 45.3740. 2/ Includes Afghanistan's SDR holdings.

3/ Represents a claim on the ministry of finance equal to the outstanding balance on a lender of last resort loan, as specified by a promissory note issued by the ministry.

4/ Before May 2011, includes lender of last resort assistance to a now-bankrupt bank—extended in September 2011 (Af 18.1 billion) and April 2012 (Af 19.5 billion) —subsequently assumed by the ministry of finance through issuance of a promissory note (see footnote above). 
Table 6. Islamic Republic of Afghanistan: Monetary Survey, 2008-13 1/

\begin{tabular}{|c|c|c|c|c|c|c|c|}
\hline & \multicolumn{4}{|c|}{ Solar year 2/ } & \multicolumn{3}{|c|}{ Year 2/ } \\
\hline & $2008 / 09$ & $2009 / 10$ & $2010 / 11$ & $2011 / 12$ & 2011 & 2012 & 2013 \\
\hline & Act. & Act. & Act. & Est. & Act. & Est. & Proj. \\
\hline & \multicolumn{7}{|c|}{ (In billions of Afghanis) } \\
\hline Net foreign assets & 183.9 & 223.7 & 274.3 & 353.8 & 348.9 & 401.9 & 459.9 \\
\hline Foreign assets & 221.1 & 257.7 & 302.2 & 387.7 & 381.7 & 440.5 & 505.4 \\
\hline Central bank & 187.1 & 209.9 & 252.1 & 319.5 & 314.6 & 356.2 & 397.8 \\
\hline Commercial banks & 34.1 & 47.8 & 50.1 & 68.1 & 67.1 & 84.3 & 107.6 \\
\hline Foreign liabilities & -37.2 & -34.0 & -27.9 & -33.8 & -32.8 & -38.6 & -45.5 \\
\hline Central bank & -6.7 & -17.1 & -16.6 & -18.5 & -18.4 & -20.6 & -22.4 \\
\hline Commercial banks & -30.5 & -16.9 & -11.3 & -15.3 & -14.4 & -18.1 & -23.1 \\
\hline Net domestic assets & -21.4 & 2.7 & 4.3 & -39.8 & -37.5 & -45.4 & -48.4 \\
\hline Net domestic credit & 18.8 & 32.2 & 33.4 & 15.7 & 16.6 & 12.5 & 13.3 \\
\hline Nonfinancial public sector & -31.1 & -37.5 & -49.7 & -28.1 & -25.6 & -33.0 & -36.5 \\
\hline Central bank & -29.3 & -34.7 & -46.9 & -25.1 & -24.0 & -31.3 & -34.8 \\
\hline Commercial banks & -1.7 & -2.7 & -2.7 & -3.0 & -1.6 & -1.7 & -1.8 \\
\hline Private sector $3 /$ & 49.8 & 69.7 & 83.1 & 43.7 & 42.2 & 45.6 & 49.8 \\
\hline Other items net & -40.2 & -29.5 & -29.1 & -55.5 & -54.1 & -57.9 & -61.6 \\
\hline Broad money M2 & 162.5 & 226.4 & 278.6 & 314.0 & 311.4 & 356.5 & 411.5 \\
\hline Narrow money M1 & 158.4 & 212.6 & 261.2 & 294.2 & 290.7 & 333.5 & 385.2 \\
\hline Currency outside banks & 73.8 & 92.5 & 126.3 & 141.2 & 141.2 & 168.2 & 194.9 \\
\hline Currency in circulation & 76.8 & 98.7 & 132.4 & 148.2 & 148.4 & 176.1 & 204.0 \\
\hline Currency held by banks & 3.0 & 6.1 & 6.2 & 7.0 & 7.1 & 7.9 & 9.1 \\
\hline Demand deposits & 84.5 & 120.1 & 134.9 & 153.1 & 149.5 & 165.3 & 190.3 \\
\hline \multirow[t]{2}{*}{ Other deposits } & 4.2 & 13.7 & 17.4 & 19.8 & 20.7 & 22.9 & 26.4 \\
\hline & \multicolumn{7}{|c|}{ (12-month percentage change) } \\
\hline M2 & 35.9 & 39.3 & 23.1 & 12.7 & 21.3 & 14.5 & 15.4 \\
\hline M1 & 37.8 & 34.3 & 22.8 & 12.7 & 21.0 & 14.7 & 15.5 \\
\hline Currency outside banks & 28.4 & 25.3 & 36.4 & 11.8 & 20.8 & 19.1 & 15.8 \\
\hline \multirow[t]{2}{*}{ Net credit to the private sector $3 /$} & 29.8 & 39.7 & 19.3 & -47.4 & -49.8 & 7.9 & 9.3 \\
\hline & \multicolumn{7}{|c|}{ (In percent of GDP) } \\
\hline M2 & 30.5 & 36.8 & 38.2 & 35.9 & 36.8 & 35.5 & 36.1 \\
\hline M1 & 29.7 & 34.6 & 35.8 & 33.6 & 34.4 & 33.2 & 33.8 \\
\hline \multicolumn{8}{|l|}{ Memorandum items: } \\
\hline M2 velocity & 3.3 & 2.7 & 2.6 & 2.8 & 2.7 & 2.8 & 2.8 \\
\hline Reserve money multiplier & 1.5 & 1.8 & 1.8 & 1.8 & 1.8 & 1.8 & 1.8 \\
\hline \multicolumn{8}{|l|}{ Banking sector } \\
\hline Loan dollarization (percent) & 73.3 & 74.4 & 73.1 & 76.5 & 76.5 & 76.5 & 76.5 \\
\hline Deposit dollarization (percent) & 58.7 & 67.5 & 65.5 & 66.6 & 66.6 & 66.6 & 66.6 \\
\hline Currency-to-deposit ratio (percent) & 86.6 & 73.8 & 86.9 & 85.7 & 87.2 & 93.6 & 94.1 \\
\hline Loans-to-deposit ratio (percent) & 56.2 & 52.1 & 54.6 & 25.3 & 24.8 & 24.2 & 23.0 \\
\hline Net open forex position & $\ldots$ & $\ldots$ & $\ldots$ & $\ldots$ & $\ldots$ & $\ldots$ & $\ldots$ \\
\hline
\end{tabular}

Source: Afghan authorities; and Fund staff estimates and projections.

$1 /$ End of period. Data underlying the survey are not fully consistent because the central bank and the public banks use the solar calendar, while commercial banks use the Gregorian calendar.

2/ Through 2011/12, the fiscal year corresponded to the Afghan calendar year, which is based on the solar year running from March 21 - March 20, in most years. In 2012, the authorities are transitioning to a fiscal year that is closer aligned with the Gregorian calendar year and runs December 22 - December 21, in most years. (Accordingly, the interim FY2012 has only 9 months.) In this table, 2011 and 2012 are recalculated to full years for comparison.

3/ The decline in 2012 reflects a write-down of bad loans of a bankrupt bank. 
Table 7. Islamic Republic of Afghanistan: Balance of Payments, 2010-2014

\begin{tabular}{|c|c|c|c|c|c|c|}
\hline & \multicolumn{2}{|c|}{ Solar year $1 /$} & \multicolumn{4}{|c|}{ Year 1/ } \\
\hline & $\begin{array}{r}2010 / 11 \\
\text { Prel. }\end{array}$ & $\begin{array}{r}2011 / 12 \\
\text { Est. }\end{array}$ & $\begin{array}{r}2011 \\
\text { Est. }\end{array}$ & $\begin{array}{l}2012 \\
\text { Proj. }\end{array}$ & $\begin{array}{l}2013 \\
\text { Proj. }\end{array}$ & $\begin{array}{l}2014 \\
\text { Proj. }\end{array}$ \\
\hline & \multicolumn{6}{|c|}{ (In millions of U.S. dollars, unless otherwise indicated) } \\
\hline Current account (including grants) & 618 & 608 & 654 & 346 & 117 & -193 \\
\hline Current account (excluding grants) & $-7,105$ & $-7,753$ & $-7,360$ & $-8,910$ & $-9,481$ & $-9,395$ \\
\hline Trade balance & $-7,070$ & $-7,734$ & $-7,358$ & $-8,875$ & $-9,512$ & $-9,410$ \\
\hline Exports of goods (f.o.b.) 2/ & 2,827 & 2,893 & 2,840 & 2,785 & 2,679 & 2,873 \\
\hline Imports of goods (f.o.b.) & $-9,897$ & $-10,626$ & $-10,199$ & $-11,660$ & $-12,191$ & $-12,283$ \\
\hline Services and income, net & -403 & -414 & -390 & -444 & -398 & -431 \\
\hline Of which: Interest due & -11 & -12 & -12 & -10 & -15 & -73 \\
\hline Current transfers & 8,091 & 8,756 & 8,402 & 9,665 & 10,027 & 9,649 \\
\hline Public (program grants) & 7,723 & 8,361 & 8,014 & 9,257 & 9,598 & 9,203 \\
\hline Private & 368 & 395 & 388 & 409 & 429 & 446 \\
\hline Financial account & 351 & 241 & 234 & 158 & 424 & 638 \\
\hline Foreign direct investment & 313 & 316 & 315 & 321 & 593 & 802 \\
\hline Official loans (net) 3/ & 133 & 156 & 27 & 59 & 106 & 109 \\
\hline Disbursement & 134 & 158 & 29 & 61 & 119 & 135 \\
\hline Amortization due & -1 & -2 & -2 & -2 & -13 & -26 \\
\hline Other portfolio flows & -95 & -231 & -108 & -222 & -275 & -273 \\
\hline Of which: Commercial banks & -117 & -276 & -211 & -287 & -397 & -357 \\
\hline Errors and omissions & -93 & -141 & 128 & 0 & 0 & 0 \\
\hline Overall balance & 876 & 709 & 1,016 & 505 & 541 & 445 \\
\hline Financing & -876 & -709 & $-1,016$ & -505 & -541 & -445 \\
\hline Central bank's gross reserves, '-' = accumulation & -876 & -727 & $-1,035$ & -538 & -565 & -464 \\
\hline Use of Fund resources (net) & 0 & 19 & 19 & 33 & 24 & 19 \\
\hline Exceptional financing & 0 & 0 & 0 & 0 & 0 & 0 \\
\hline Financing gap & 0 & 0 & 0 & 0 & 0 & 0 \\
\hline \multicolumn{7}{|l|}{ Memorandum items: } \\
\hline Gross international reserves, central bank & 5,403 & 6,234 & 6,208 & 6,721 & 7,286 & 7,750 \\
\hline (Import coverage) 4/ & 6.4 & 6.5 & 6.7 & 6.8 & 7.3 & 7.7 \\
\hline \multirow[t]{2}{*}{ (Relative to external debt service) } & 435 & 468 & 474 & 421 & 181 & 65.2 \\
\hline & \multicolumn{6}{|c|}{ (In percent of GDP, unless otherwise indicated) } \\
\hline Trade balance & -44.4 & -42.2 & -40.6 & -44.4 & -43.2 & -39.6 \\
\hline Current account balance, including grants & 3.9 & 3.3 & 3.6 & 1.7 & 0.5 & -0.8 \\
\hline Excluding grants & -44.6 & -42.3 & -40.7 & -44.6 & -43.0 & -39.6 \\
\hline Total debt service (percent of exports) 4/ & 1.1 & 1.1 & 1.1 & 0.9 & 1.5 & 2.0 \\
\hline Total debt stock & 8.2 & 6.6 & 6.9 & 6.7 & 6.6 & 6.7 \\
\hline
\end{tabular}

Source: Afghan authorities; and Fund staff estimates and projections.

1/ Through 2011/12, the fiscal year corresponded to the Afghan calendar year, which is based on the solar year running from March 21 to March 20, in most years. In 2012, the authorities are transitioning to a fiscal year that is closer aligned with the Gregorian calendar year and runs December 22 - December 21, in most years. (Accordingly, the interim FY2012 has only 9 months.) In this table, 2011 and 2012 are recalculated to full years for comparison.

2/ Excludes opium exports; includes official exports, estimates of smuggling, reexport, and sales to nonresidents.

3/ Excluding IMF credit.

4/ Excluding reexport-related flows. 
Table 8. Islamic Republic of Afghanistan:

Proposed Schedule of Reviews and Disbursements Under the ECF Arrangement

\begin{tabular}{|c|c|c|c|}
\hline \multirow[b]{2}{*}{ Date } & \multicolumn{2}{|c|}{ Amount of disbursement } & \multirow[b]{2}{*}{ Conditions } \\
\hline & Millions of SDRs & Percent of quota $1 /$ & \\
\hline November 23, 2011 & 12.0 & 7.4 & Approval of arrangement 2/ \\
\hline June 20, 2012 & 12.0 & 7.4 & $\begin{array}{l}\text { First review and March 19, } 2012 \\
\text { performance criteria }\end{array}$ \\
\hline December 20, 2012 & 12.0 & 7.4 & $\begin{array}{l}\text { Second review and September 21, } 2012 \\
\text { performance criteria }\end{array}$ \\
\hline March 20, 2013 & 12.0 & 7.4 & $\begin{array}{l}\text { Third review and December 20, } 2012 \\
\text { performance criteria }\end{array}$ \\
\hline September 20, 2013 & 12.0 & 7.4 & $\begin{array}{l}\text { Fourth review and June 21, } 2013 \\
\text { performance criteria }\end{array}$ \\
\hline March 20, 2014 & 12.0 & 7.4 & $\begin{array}{l}\text { Fifth review and December 21, } 2013 \\
\text { performance criteria }\end{array}$ \\
\hline September 20, 2014 & 13.0 & 8.0 & $\begin{array}{l}\text { Sixth review and June } 21,2014 \\
\text { performance criteria }\end{array}$ \\
\hline Total & 85.0 & 52.5 & \\
\hline
\end{tabular}

Source: Fund staff estimates.

$1 /$ Percentages do not add up to the total due to rounding.

2/ The arrangement was approved on November 14, 2011 and the first disbursement drawn on the date indicated. 
Table 9. Islamic Republic of Afghanistan:

External Financing Requirement and Sources, 2010-14

\begin{tabular}{|c|c|c|c|c|}
\hline & \multicolumn{4}{|c|}{ Year 1/ } \\
\hline & $\begin{array}{c}2011 \\
\text { Prel. }\end{array}$ & $\begin{array}{c}2012 \\
\text { Proj. }\end{array}$ & $\begin{array}{l}2013 \\
\text { Proj. }\end{array}$ & $\begin{array}{c}2014 \\
\text { Proj. }\end{array}$ \\
\hline & \multicolumn{3}{|c|}{ (In millions of U.S. dollars) } & \\
\hline I. Total financing requirement & 8,396 & 9,454 & 10,071 & 9,905 \\
\hline Current account (excluding grants) & 7,360 & 8,910 & 9,481 & 9,395 \\
\hline Amortization & 2 & 6 & 25 & 46 \\
\hline Of which: IMF & 0 & 4 & 13 & 20 \\
\hline Prepayment of debt & 0 & 0 & 0 & 0 \\
\hline Change in reserves (increase $=+$ ) & 1,035 & 538 & 565 & 464 \\
\hline Reduction in arrears & 0 & 0 & 0 & 0 \\
\hline II. Available financing & 8,396 & 9,454 & 10,071 & 9,905 \\
\hline Current transfers & 8,014 & 9,257 & 9,598 & 9,203 \\
\hline Foreign direct investment & 315 & 321 & 593 & 802 \\
\hline Official medium- and long-term loans & 29 & 61 & 119 & 135 \\
\hline IMF disbursements & 19 & 37 & 37 & 38 \\
\hline Debt forgiveness & 0 & 0 & 0 & 0 \\
\hline Debt rescheduling & 0 & 0 & 0 & 0 \\
\hline Other items, net $2 /$ & 19 & -222 & -275 & -273 \\
\hline III. Financing gap (I-II) & 0 & 0 & 0 & 0 \\
\hline
\end{tabular}

Sources: Afghan authorities; and Fund staff estimates and projections.

$1 /$ Through 2011/12, the fiscal year corresponded to the Afghan calendar year, which is based on the solar year running from March 21 to March 20, in most years. In 2012, the authorities are transitioning to a fiscal year that is closer aligned with the Gregorian calendar year and runs December 22 - December 21, in most years. (Accordingly, the interim FY2012 has only 9 months.) In this table, 2011 and 2012 are recalculated to full years for comparison.

2/ Includes commercial banks' accumulation of gross reserves 


\section{APPENDIX I. ISLAMIC REPUBLIC OF AFGHANISTAN: LETTER OF INTENT}

June 18, 2012

Ms. Christine Lagarde

Managing Director

International Monetary Fund

Washington, DC 20431

Dear Ms. Lagarde:

We would like to thank the International Monetary Fund for supporting our economic program under the Extended Credit Facility (ECF) arrangement, approved by the Executive Board on November 14, 2011. The program has guided our macroeconomic policy formulation and structural reforms over the last six months and has helped us to manage some of the key challenges facing Afghanistan's economy, though progress in important areas such as asset recovery from Kabul Bank has been difficult and slow. Looking ahead, our program will continue to focus on safeguarding and developing the financial sector, strengthening economic governance, and moving toward fiscal sustainability.

We are pleased to inform you that we have made progress on our structural reform agenda. Albeit with delays, we have completed five out of six structural benchmarks for the first review (Table 1). At New Kabul Bank, we have closed 25 branches to improve efficiency, we have appointed a privatization advisor, and the economic committee of the cabinet approved privatization of the bank. We have submitted value-added tax legislation for a formal judicial review, are incorporating Fund's comments to the recently prepared amendments to the law on state-owned enterprises - however, amendments to the company law to strengthen oversight and control over state-owned corporation will take until the end of the year - and rolled out the new border management model to additional border crossing points on time. The ministry of finance and Da Afghanistan Bank (DAB) have signed a memorandum of understanding on central bank capitalization, and an external auditor for DAB's 2011/12 financial statements has been appointed. Finally, a national economic crimes strategy was approved and included in the National Priority Program "Justice for All."

We have also made progress on asset recovery from Kabul Bank since April. Given the, in general, poor payment performance of the existing legally binding agreements, we decided to discontinue this option for shareholders or related parties to repay their obligations to the receivership. Instead, President Karzai has given shareholders and related parties until June 4, 2012 to repay their debts in full or be referred to a special tribunal. Based on the decree, we have initiated an asset recovery process for each of the beneficiaries (individuals or corporations) identified in a forensic audit report for the amounts specified therein. Only regular borrowers who remain current on their obligations can continue to repay on their original schedule. 
As of June 4, 2012, cash recoveries from Kabul Bank beneficiaries amount to US\$128 million out of US\$935 million identified assets transferred to the receiver-an additional US\$54 million since November 2011. The presidential decree expedited the cash recovery process by bringing in US\$30 million since its issuance on April 4, 2012. In addition, we have taken over properties with an estimated value of US\$44 million in Dubai, and properties with a book value of US\$146 million in Afghanistan. No beneficiary identified in the forensic audit report has paid in full. The two main architects of the fraud have been referred to the special tribunal, and the remaining beneficiaries will be dealt with by the Financial Dispute Resolution Committee, and, if warranted, could be referred to the special tribunal at a later date. In the case of Pamir Airways, Gulbahar Towers, and the Zahira oil tank farm, assets have been transferred to or are under the full legal control of the receiver. In order to assist our investigations and our efforts to recover assets outside of Afghanistan, we have issued mutual legal assistance requests to the United Arab Emirates, and will issue similar requests to other relevant foreign jurisdictions shortly.

We also met all but one of the end-March 2012 quantitative performance criteria. DAB contained reserve money growth to below the program ceiling while accumulating net international reserves by more than projected under the program. The government achieved its social and other priority spending objectives. We did not contract new nonconcessional external debt or accumulate external payment arrears.

However, reflecting trade disruptions at the border with Pakistan and problems in revenue administration, we missed our revenue target by a small margin. This shortfall also translated into a higher than programmed operating deficit of the government, an indicative target. Since revenue mobilization remains one of the government's top priorities, we are taking immediate measures to address the underlying problems that are under our control. Specifically, in fiscal year 2012, we have not renewed and will not renew concessions for certain taxpayer groups that have undermined our revenue base in the past. This measure is also important to ensure the success of the value-added tax introduction.

Accordingly, we would like to hereby kindly request the completion of the first review, the second disbursement of SDR 12 million, and a waiver for nonobservance of the quantitative performance criterion on revenue collection by the central government given that the shortfall was minor and that we are taking corrective action. Completion of the review will allow us to consolidate reform gains while providing necessary confidence to our development partners.

Our reform agenda ahead is ambitious. Fiscal and monetary policies will continue to be geared toward revenue generation and containing inflation. Moreover, in the coming months, we will take necessary steps to contain emerging risks in the financial sector, including tightening capital requirements and strengthening supervision. We will also submit to the 
ministry of justice and subsequently to parliament amendments and new laws critical to building good economic institutions, including in the areas of central banking, commercial banking, VAT, debt securities, public enterprises, anti-money laundering and countering the financing of terrorism, and establish an interagency framework for the implementation of these laws.

Our policies for the next 12 months are laid out in the attached Memorandum of Economic and Financial Policies (MEFP) and the Technical Memorandum of Understanding (TMU). The program will be monitored through quantitative performance criteria and indicative targets (Table 2) as well as structural benchmarks (Table 1). We also request that the ECF arrangement be rephased to make it consistent with the new Afghan fiscal year (Table 3).

We believe that the policies set forth in the MEFP are adequate to achieve the objectives of the program, but stand ready to take any further measures that may become appropriate for this purpose. We will consult with the Fund on the adoption of these measures and in advance of any revision to the policies contained in the MEFP, in accordance with the Fund's policies on such consultation. We will remain in close consultation with Fund staff and provide timely information necessary for monitoring economic developments and implementation of policies under the ECF-supported program.

Sincerely yours,

$/ \mathrm{s} /$

Omar Zakhilwal

Minister of Finance
$/ \mathrm{s} /$

Noorullah Delawari

Governor Da Afghanistan Bank

Tables:

Table 1. Islamic Republic of Afghanistan: Prior Actions and Structural Benchmarks

Table 2a-b. Islamic Republic of Afghanistan: Quantitative Targets

Table 3. Rephasing and Change to the Schedule of Reviews

Attachments:

Memorandum of Economic and Financial Policies

Technical Memorandum of Understanding 


\section{Table 1. Islamic Republic of Afghanistan: Prior Actions and Structural Benchmarks}

\section{$\begin{array}{lll}\text { Measure } & \text { Rationale Target Date Status as of June } 10\end{array}$}

\section{Structural Benchmarks for the First Review}

In consultations with Fund staff, the ministry of finance and the central bank will agree, in a memorandum of understanding, on the central bank's capitalization needs and a schedule for recapitalizing the central bank as needed.

Submit VAT law-consistent with IMF advice-aimed at raising the revenue-toGDP ratio to the ministry of justice ("Taqnin") for review with a view to submitting it to parliament by endDecember 2012.

Submit to the ministry of justice ("Taqnin") legislation bringing public enterprises under effective monitoring and oversight of the ministry of finance.

Roll out to at least two additional Border Crossing Points the new Border Management Model piloted at Hairatan Border Crossing Point. The model is based on a 'two-agency' approach which restricts border presence to the Afghan Border Police and the Afghan Customs Department. Under this model, the ABP will continue to fulfill immigration and security responsibilities, with ACD fulfilling all other commercial and trade-related duties.

Reduce number of branches at bridge bank (New Kabul Bank) by 20 according to the updated business plan.
Financial sector Endstability, central December

bank 2011 independence and ability to conduct monetary policy

\section{Support fiscal} sustainability

EndDecember 2011

Support fiscal

End-March sustainability

2012

macroeconomic stability by enhancing controls and reducing fiscal risks

\section{Improve} efficiency and lower corruption 2012

End-March Completed in April 2012.

Minimize fiscal costs
End-March 2012
Completed in June 2012.

Completed in December 2011.

Draft amendments to the state-owned enterprise law have been shared with staff, and we will incorporate comments received. State-owned corporations will be addressed later this year (see new end-December 2012 structural benchmark).

Completed. 25 branches have been closed as of end-January 2012. 
In line with our desire to protect the financial system from abuse, the government will produce a roadmap with a strategy to build capacity and improve the institutional framework to respond promptly to economic crime.

This roadmap will address issues related to the prosecution system to reinforce its independence to ensure accountability for economic crimes and build capacity for investigation and prosecution of these crimes as well as determine the most appropriate institutional framework going forward.
Financial sector End-March The strategy was included stability and 2012 in the "Justice for All" integrity, reduce moral hazard National Priority Program on May 15, 2012.

\section{Structural Benchmarks for the Second Review}

The economic committee of the cabinet Advance Kabul End-August will approve the privatization plan for Bank resolution 2012 New Kabul Bank prepared by the privatization advisor in consultation with Fund staff. The plan includes clear criteria for bidders, precise determination of what is being sold, the procedures for sale, and public communication strategy (MEFP paragraph 25). 
The bridge bank (New Kabul Bank) will be offered for sale in a transparent way, involving a pre-qualification process to ensure that controlling shareholders, beneficial owners, directors and management of intending bidders are fit and proper (which implies, inter alia, that an intending bidder is subject to a high standard of corporate governance, including risk management and internal controls, and that an intending bidder is in a sound financial and risk management state), and that the intending bidder controls adequate resources and has the necessary capacity and capability to ensure the ongoing provision of the relevant salary payments services. There will be a request for expressions of interest locally and internationally for the sale of the bridge bank. Interested parties will be given four weeks to respond with intention to participate in the bid. Once the central bank has vetted potential buyers, the ministry of finance will give them a deadline for sealed bids (MEFP paragraph 25).

The independent Monitoring and Evaluation Commission will conduct an indepth public inquiry to examine the events leading to the Kabul Bank crisis, starting with the inception of the bank, and look into the operations of the bank, activities of its shareholders, the role of supervisory and auditing bodies, and the subsequent effectiveness of the government and the criminal justice system in dealing with any crimes committed (MEFP paragraph 11).
Advance Kabul End-

Bank resolution

September 2012
Delayed from end-June

2012 because it took longer than expected to appoint a privatization advisor. The advisor is now in place.
Strengthen End-

financial sector September stability, transparency 
Measure
Rationale Target Date

Status as of June 10

\begin{tabular}{|c|c|c|c|}
\hline $\begin{array}{l}\text { Submit to parliament the amended or new } \\
\text { banking law, prepared in consultation with } \\
\text { the Fund that will strengthen provisions on } \\
\text { corporate governance, capital, large } \\
\text { exposures, related parties, consolidated } \\
\text { supervision, early intervention, } \\
\text { enforcement, and bank resolution, where } \\
\text { appropriate in line with the Basel Core } \\
\text { Principles. Specifically, the law should } \\
\text { enable us to enforce upon a bank - going } \\
\text { concern - all necessary resolution } \\
\text { measures and strengthen corporate } \\
\text { governance requirements for banks } \\
\text { (including fit and proper requirements as } \\
\text { set by the Financial Action Task Force } \\
\text { standards) (MEFP paragraph 21). }\end{array}$ & $\begin{array}{l}\text { Promote } \\
\text { financial sector } \\
\text { stability by } \\
\text { strengthening } \\
\text { independence } \\
\text { of bank } \\
\text { regulators, } \\
\text { reducing risks } \\
\text { from bank } \\
\text { lending, and } \\
\text { ensuring swift } \\
\text { processes for } \\
\text { bank resolution }\end{array}$ & $\begin{array}{l}\text { End- } \\
\text { September } \\
2012\end{array}$ & $\begin{array}{l}\text { Draft law is being finalized } \\
\text { by DAB and needs to be } \\
\text { sent to the ministry of } \\
\text { justice next. }\end{array}$ \\
\hline $\begin{array}{l}\text { Strengthen our AML/CFT regime by } \\
\text { implementing an action plan based on } \\
\text { the recommendations of the February } \\
2011 \text { assessment by: (i) submitting } \\
\text { amendments to the AML/CFT law to } \\
\text { parliament as necessary; (ii) increasing the } \\
\text { capacity of FinTRACA, including by hiring } \\
\text { additional staff as needed; (iii) expanding } \\
\text { MSP registration and implementation of } \\
\text { reporting to MSPs in areas currently } \\
\text { inaccessible for security reasons if and } \\
\text { when the security situation allows; and (iv) } \\
\text { enforcing MSP reporting by dedicated } \\
\text { software in all reporting areas where it is } \\
\text { technically and logistically feasible (MEFP } \\
\text { paragraph 30). }\end{array}$ & $\begin{array}{l}\text { Promote } \\
\text { financial sector } \\
\text { stability and } \\
\text { integrity and } \\
\text { strengthen } \\
\text { AML/CFT } \\
\text { framework }\end{array}$ & $\begin{array}{l}\text { End- } \\
\text { September } \\
2012\end{array}$ & \\
\hline $\begin{array}{l}\text { Submit legislation to the ministry of justice } \\
\text { ("Taqnin") for review with a view to } \\
\text { submitting it to parliament by end-March } \\
2013 \text { for the introduction of marketable } \\
\text { debt instruments by the ministry of } \\
\text { finance (MEFP paragraph 39). }\end{array}$ & $\begin{array}{l}\text { Develop } \\
\text { financial } \\
\text { markets }\end{array}$ & $\begin{array}{l}\text { End- } \\
\text { September } \\
2012\end{array}$ & $\begin{array}{l}\text { We have delayed } \\
\text { submission to parliament } \\
\text { to allow for wide } \\
\text { consultation of the law } \\
\text { with all relevant } \\
\text { stakeholders. }\end{array}$ \\
\hline
\end{tabular}




\begin{abstract}
Measure
The Supreme Council of Da Afghanistan

Bank to approve strategies for banks

that fall short of the minimum paid-up

capital to be merged or closed, with the process starting no later than December 2012 (MEFP paragraph 23).

\section{Structural Benchmarks for the Third Review}

Following the approval by the cabinet submit draft law on VAT to the parliament. The draft legislation will be prepared in consultation with Fund staff, limit exemptions, and require that new exemptions be introduced only through parliamentary approval in the VAT law (MEFP paragraph 34).
\end{abstract}

Rationale Target Date

Status as of June 10
Financial stability

$\begin{array}{ll}\text { Fiscal } & \text { End- } \\ \text { sustainability, } & \text { December } \\ \text { revenue } & 2012 \\ \text { mobilization } & \end{array}$

$\begin{array}{ll}\text { VAT } & \text { End- } \\ \text { implementation; } & \text { December } \\ \text { tax } & 2012 \\ \text { administration } & \\ \text { and revenue } & \\ \text { collection } & \end{array}$

End-

September

2012
The Afghanistan Revenue Department within the ministry of finance to identify taxpayers in the medium taxpayer office and the small taxpayer office whose turnover exceeds the large taxpayer office threshold and revisit the thresholds for the large taxpayer office and the medium taxpayer office with a view to having an efficient allocation of taxpayers across the three offices. The findings and actions to be summarized in a report (MEFP paragraph 35).

Submit to the ministry of justice ("Taqnin") legislation bringing state-owned corporations under effective monitoring and oversight of the ministry of finance, including financial reporting to the ministry of finance and ministry of finance approving financial plans on an annual basis. The draft legislation will be prepared in consultation with Fund staff (MEFP paragraph 40).

Approval by the Supreme Council of Da Afghanistan Bank of the new organizational structure of the Financial Supervision Department developed in consultation with Fund staff (MEFP paragraph 22).

\author{
Corporate End- \\ governance; December \\ transparency; 2012 \\ sound public \\ financial \\ management, \\ fiscal \\ sustainability \\ (by reducing \\ fiscal risks)
}

Strengthening supervision;

EndDecember 2012 


$\begin{array}{lll}\text { Revise central bank regulations as well } & \text { Improve } & \text { End- } \\ \begin{array}{l}\text { as prepare and issue informational } \\ \text { circulars to ensure consistency with the }\end{array} & \text { financial } & \text { December } \\ \text { revised banking law (MEFP paragraph 21). } & & 2012\end{array}$

\section{Structural Benchmarks for the Fourth Review}

\begin{abstract}
Establish a senior official-led interagency steering committee (Economic Crimes Task Force) to oversee the passage and implementation of the new banking law, the anti-money laundering and terrorist financing laws and their provisions, and to function as a coordinating body for the implementation of the Economic Crimes Strategy and its action items (MEFP paragraph 29).
\end{abstract}

Submit to the ministry of justice ("Taqnin") amendments to the income tax law, minerals law, oil and gas (hydrocarbons) law and customs code-developed in consultation with Fund staff-related to the development of a sound and robust

natural resources fiscal regime to attract investment and ensure the government has a reasonable share of the economic rent (MEFP paragraph 37).

\section{Sign a memorandum of understanding between Financial Transactions and Reports Analysis Center of Afghanistan, the Financial Supervision Department of DAB, and competent law enforcement authorities on information sharing as described in the attached MEFP (paragraph 30).}

\section{Financial integrity, End-March 2013} financial development

Fiscal sustainability, revenue mobilization

\section{End-June}

2013

\section{Financial End-June integrity, 2013 financial development}




\begin{tabular}{|c|c|c|c|}
\hline Measure & Rationale & Target Date & Status as of June 10 \\
\hline $\begin{array}{l}\text { Submit to parliament amendments to } \\
\text { the central bank legislation-drafted in } \\
\text { consultation with Fund staff-- } \\
\text { implementing the memorandum of } \\
\text { understanding on the central bank's } \\
\text { capitalization, that aligns the provisions on } \\
\text { the netting and allocation of net income } \\
\text { with international good practice (MEFP } \\
\text { paragraph } 31 \text { ). }\end{array}$ & $\begin{array}{l}\text { Central bank } \\
\text { capitalization, } \\
\text { macroeconomic } \\
\text { stability }\end{array}$ & $\begin{array}{l}\text { End-June } \\
2013\end{array}$ & \\
\hline
\end{tabular}


Table 2a. Islamic Republic of Afghanistan: Quantitative Targets, 2011/12

(cumulatively from the beginning of the fiscal year, unless otherwise indicated)

\begin{tabular}{|c|c|c|c|c|c|c|c|c|c|}
\hline & \multirow{3}{*}{$\begin{array}{r}\text { Mar. 20, } 2011 \\
\text { Stocks }\end{array}$} & \multicolumn{8}{|c|}{ Fiscal Year 2011/12 } \\
\hline & & \multirow{2}{*}{$\begin{array}{r}\text { June 21, } 2011 \\
\text { Actual }\end{array}$} & \multirow{2}{*}{$\begin{array}{r}\text { Sep. 22, } 2011 \\
\text { Actual }\end{array}$} & \multicolumn{3}{|c|}{ Dec. 21, 2011} & \multicolumn{3}{|c|}{ Mar. 19, 2012} \\
\hline & & & & $\begin{array}{r}\text { Indicative } \\
\text { targets }\end{array}$ & Adj. & Act. & $\begin{array}{r}\text { Performance } \\
\text { criteria }\end{array}$ & $\begin{array}{l}\text { Est. } \\
\text { adj. }\end{array}$ & Prel. \\
\hline & \multicolumn{9}{|c|}{ (In billions of Afghanis) } \\
\hline Floor on revenues of the central government & $\ldots$ & 21.7 & 46.1 & 71.6 & $\ldots$ & 69.3 & 98.8 & $\ldots$ & 97.3 \\
\hline Indicative target (ceiling) on currency in circulation & 132.4 & 1.3 & 11.9 & 16.6 & $\ldots$ & 15.9 & 23.6 & $\ldots$ & 15.7 \\
\hline Ceiling on net central bank credit to the central government & -46.9 & 29.1 & 26.2 & 17.8 & 37.0 & 26.8 & 24.8 & 38.0 & 25.8 \\
\hline $\begin{array}{l}\text { Indicative target (ceiling) on the operating budget deficit } \\
\text { of the government, excluding grants }\end{array}$ & $\ldots$ & 1.4 & 12.9 & 33.8 & 33.8 & 28.8 & 52.6 & 52.6 & 53.0 \\
\hline Indicative target (floor) on social and other priority spending & $\ldots$ & 4.5 & 9.0 & 13.5 & $\ldots$ & 12.7 & 18.0 & $\ldots$ & 18.2 \\
\hline \multirow[t]{2}{*}{ Ceiling on reserve money } & 151.0 & 15.4 & 17.3 & 15.0 & $\ldots$ & 19.2 & 28.4 & $\ldots$ & 24.3 \\
\hline & \multicolumn{9}{|c|}{ (In millions of U.S. dollars) } \\
\hline Floor on net international reserves of $D A B$ & 5,099 & 454 & 519 & 572 & 182 & 704 & 630 & 363 & 665 \\
\hline $\begin{array}{l}\text { Ceiling on contracting or guaranteeing new medium- and long-term } \\
\text { nonconcessional external debt by the public sector } 2 /\end{array}$ & $\ldots$ & 0.0 & 0.0 & 0.0 & $\ldots$ & 0.0 & 0.0 & $\ldots$ & 0.0 \\
\hline Ceiling on short-term external debt owed or guaranteed by the public sector $2 /$ & $\ldots$ & 0.0 & 0.0 & 0.0 & $\ldots$ & 0.0 & 0.0 & $\ldots$ & 0.0 \\
\hline New external payments arrears, excluding interest on preexisting arrears $2 /$ & $\cdots$ & 0.0 & 0.0 & 0.0 & $\ldots$ & 0.0 & 0.0 & $\ldots$ & 0.0 \\
\hline $\begin{array}{l}\text { Ceiling on lending from state-owned banks or the central bank to public enterprises in } \\
\text { need of restructuring or government guaranteeing borrowing by these enterprises } 2 /\end{array}$ & $\cdots$ & 0.0 & 0.0 & 0.0 & $\cdots$ & 0.0 & 0.0 & $\ldots$ & 0.0 \\
\hline \multicolumn{10}{|l|}{ Reference projections for the adjustors } \\
\hline & & & & \multicolumn{3}{|c|}{ Deviation } & \multicolumn{3}{|c|}{ Deviation } \\
\hline Core budget development spending & $\ldots$ & 5.3 & 16.9 & 33.5 & -1.6 & 31.9 & 53.4 & 0.0 & 53.4 \\
\hline External financing of the core budget and sale or transfers of nonfinancial assets & $\ldots$ & 21.7 & 41.9 & 85.4 & -18.4 & 67.0 & 115.3 & -10.7 & 104.6 \\
\hline Asset recovery from banking sector institutions in liquidation & $\ldots$ & 0.0 & 1.0 & 1.8 & -0.8 & 1.0 & 3.5 & -2.5 & 1.0 \\
\hline Recapitalization, net of profit distribution and revaluation of MoF securities at DAB & $\ldots$ & 0.0 & 0.0 & 0.0 & 0.0 & 0.0 & 0.0 & 0.0 & 0.0 \\
\hline Externally financed expenditures transferred to the core operating budget & $\ldots$ & 0.0 & 0.0 & 0.0 & 0.0 & 0.0 & 0.0 & 0.0 & 0.0 \\
\hline
\end{tabular}

Source: Afghan authorities

1/ The performance criteria and indicative targets under the program, and their adjustors, are defined in the Technical Memorandum of Understanding.

$2 /$ These targets apply on a continuous basis. 
Table 2b. Islamic Republic of Afghanistan: Quantitative Targets, 2012-13

(cumulatively from the beginning of the respective fiscal year, unless otherwise indicated)

\begin{tabular}{|c|c|c|c|c|c|c|c|c|c|c|}
\hline & \multirow{3}{*}{$\begin{array}{r}\text { Mar. 19, } 2012 \\
\text { Stocks }\end{array}$} & \multicolumn{5}{|c|}{ Fiscal Year 2012 (interim, 9-months fiscal year) } & \multicolumn{4}{|c|}{ Fiscal Year 2013} \\
\hline & & \multicolumn{2}{|c|}{$\begin{array}{c}\text { June } 20,2012 \\
\text { Indicative targets }\end{array}$} & \multicolumn{2}{|c|}{$\begin{array}{c}\text { Sep. 21, } 2012 \\
\text { Performance criteria }\end{array}$} & \multirow{2}{*}{$\begin{array}{r}\text { Dec. 20, } 2012 \\
\text { Performance } \\
\text { criteria }\end{array}$} & \multirow{2}{*}{$\begin{array}{r}\text { Mar. 20, } 2013 \\
\text { Indicative } \\
\text { targets }\end{array}$} & \multirow{2}{*}{$\begin{array}{r}\text { June } 21,2013 \\
\text { Performance } \\
\text { criteria }\end{array}$} & \multirow{2}{*}{$\begin{array}{r}\text { Sep. 22, } 2013 \\
\text { Proj. }\end{array}$} & \multirow{2}{*}{$\begin{array}{r}\text { Dec. 21, } 2013 \\
\text { Proj. }\end{array}$} \\
\hline & & Prog. & Proj. & Prog. & Rev. & & & & & \\
\hline & \multicolumn{10}{|c|}{ (In billions of Afghanis) } \\
\hline Floor on revenues of the central government & $\ldots$ & 23.8 & 23.8 & 50.4 & 50.4 & 85.1 & 28.2 & 59.7 & 92.6 & 129.0 \\
\hline Indicative target (ceiling) on currency in circulation & 148.2 & 1.5 & 4.7 & 12.7 & 18.6 & 28.0 & 3.5 & 13.9 & 20.9 & 27.8 \\
\hline Ceiling on net central bank credit to the central government & -21.1 & -9.5 & -11.4 & -4.4 & -7.7 & -4.8 & -2.2 & -1.3 & -2.5 & -2.3 \\
\hline $\begin{array}{l}\text { Indicative target (ceiling) on the operating budget deficit } \\
\text { of the government, excluding grants }\end{array}$ & $\ldots$ & 10.1 & 10.1 & 29.2 & 29.2 & 45.3 & 16.0 & 33.9 & 52.6 & 73.3 \\
\hline Indicative target (floor) on social and other priority spending & $\cdots$ & 4.8 & 6.1 & 9.7 & 12.2 & 18.3 & 6.0 & 12.0 & 18.0 & 23.9 \\
\hline \multirow[t]{2}{*}{ Ceiling on reserve money } & 175.3 & -4.0 & 0.0 & 6.5 & 9.8 & 26.7 & 0.0 & 8.9 & 24.2 & 32.3 \\
\hline & \multicolumn{10}{|c|}{ (In millions of U.S. dollars) } \\
\hline Floor on net international reserves of $\mathrm{DAB}$ & 5,764 & 117 & 297 & 234 & 434 & 550 & 137 & 275 & 413 & 550 \\
\hline $\begin{array}{l}\text { Ceiling on contracting or guaranteeing new medium- and long-term } \\
\text { nonconcessional external debt by the public sector } 2 /\end{array}$ & $\cdots$ & 0.0 & 0.0 & 0.0 & 0.0 & 0.0 & 0.0 & 0.0 & 0.0 & 0.0 \\
\hline Ceiling on short-term external debt owed or guaranteed by the public sector $2 /$ & $\ldots$ & 0.0 & 0.0 & 0.0 & 0.0 & 0.0 & 0.0 & 0.0 & 0.0 & 0.0 \\
\hline New external payments arrears, excluding interest on preexisting arrears $2 /$ & $\cdots$ & 0.0 & 0.0 & 0.0 & 0.0 & 0.0 & 0.0 & 0.0 & 0.0 & 0.0 \\
\hline $\begin{array}{l}\text { Ceiling on lending from state-owned banks or the central bank to, or government } \\
\text { guaranteed borrowing by, public enterprises in need of restructuring } 2 /\end{array}$ & $\ldots$ & 0.0 & 0.0 & 0.0 & 0.0 & 0.0 & 0.0 & 0.0 & 0.0 & 0.0 \\
\hline Ceiling on government guarantees $2 /$ & $\cdots$ & 0.0 & 0.0 & 0.0 & 0.0 & 0.0 & 0.0 & 0.0 & 0.0 & 0.0 \\
\hline \multicolumn{11}{|l|}{$\underline{\text { Reference projections for the adjustors }}$} \\
\hline Core budget development spending & $\ldots$ & 4.2 & 4.2 & 23.9 & 23.9 & 49.6 & 15.9 & 33.7 & 52.3 & 72.9 \\
\hline External financing of the core budget and sale of nonfinancial assets & $\ldots$ & 22.8 & 25.4 & 55.7 & 58.7 & 96.2 & 33.8 & 67.8 & 105.8 & 146.2 \\
\hline Asset recovery from banking sector institutions in liquidation & $\ldots$ & 0.9 & 1.3 & 1.9 & 2.5 & 3.8 & 0.6 & 1.3 & 1.9 & 2.6 \\
\hline Recapitalization, net of dividend, and revaluation of MoF's promissory note & $\ldots$ & 0.0 & 0.0 & 0.0 & 0.0 & 0.0 & 0.0 & 0.0 & 0.0 & 0.0 \\
\hline Externally financed expenditures transferred to the core operating budget & $\ldots$ & 0.0 & 0.0 & 0.0 & 0.0 & 0.0 & 0.0 & 0.0 & 0.0 & 0.0 \\
\hline
\end{tabular}

Source: Afghan authorities

1/ The performance criteria and indicative targets under the program, and their adjustors, are defined in the Technical Memorandum of Understanding

2/ These targets apply on a continuous basis. 
Table 3. Islamic Republic of Afghanistan: Rephasing and Change to the Schedule of Reviews

\begin{tabular}{|c|c|c|c|c|c|c|}
\hline \multirow[t]{3}{*}{ Review } & \multicolumn{6}{|c|}{ Disbursement } \\
\hline & \multicolumn{2}{|c|}{ Date } & \multicolumn{2}{|c|}{ Amount 1/ } & \multicolumn{2}{|c|}{ Conditions (test dates) $2 /$} \\
\hline & Old & New & Old & New & Old & New \\
\hline Second & November 20, 2012 & December 20, 2012 & 12.0 & 12.0 & September 21, 2012 & September 21, 2012 \\
\hline Third & May 20,2013 & March 20, 2013 & 12.0 & 12.0 & March 20, 2013 & December 20, 2012 \\
\hline Fourth & November 20, 2013 & September 20, 2013 & 12.0 & 12.0 & September 22, 2013 & June 21, 2013 \\
\hline Fifth & May 20, 2014 & March 20, 2014 & 12.0 & 12.0 & March 20, 2014 & December 21, 2013 \\
\hline Sixth & November 6, 2014 & September 20, 2014 & 13.0 & 13.0 & September 22, 2014 & June 21, 2014 \\
\hline
\end{tabular}

1/ SDR million.

2/ Approval of the respective review based on performance criteria set for the indicated test dates. 


\section{Attachment I. Memorandum of Economic and Financial Policies}

1. This memorandum provides an update on our economic program supported by the IMF's Extended Credit Facility (ECF) approved the IMF's Executive Board on November 14, 2011. It reviews program implementation through early June 2012 and summarizes the government's economic objectives and policy framework for the next 12 months - a period covered by the second, the third, and fourth review. The memorandum complements our previous memorandum, attached to the letter of intent dated November 1, 2011.

\section{The policies described here are consistent with the Afghanistan National} Development Strategy (ANDS), our umbrella development agenda. Through the Kabul process, launched in 2010, we have further prioritized the ANDS through 22 National Priority Programs (NPPs) to reflect budgetary realities and provide a framework to measure results. The design, sequencing, and financing of the NPPs are currently being discussed with our development partners. The ANDS Annual Progress Report was published in September 2011 and a new progress report is being prepared.

3. The focus of our program remains broadly appropriate. We have made a number of achievements in key reform areas over the past six months, including in the areas of introducing a value-added tax (VAT) in 2014, improving the central bank's capitalization framework, and asset recovery from Kabul Bank. Looking forward, the program will focus on safeguarding and developing the financial system, further strengthening economic governance, and moving toward fiscal sustainability.

\section{RECENT DEVELOPMENTS}

4. Macroeconomic developments in 2011/12 were impacted by adverse external factors. Real GDP growth in 2011/12 is estimated at 6 percent - a slowdown from a year ago, largely due to the decline in agricultural output. Inflation slowed to single digits in 2011, but in recent months accelerated again to 11 percent year on year in March, mainly due to rising food and fuel prices. The current account, despite weak exports, has been sustained by continuing aid inflows. Consistent with the program, we have not incurred any new nonconcessional external debt. After having reached the HIPC completion point in 2010, we successfully negotiated debt forgiveness agreements with the United States and Germany, and we also received 100 percent debt relief from Saudi Arabia, Iraq, and Croatia. We continue to discuss debt restructuring and relief with both Paris Club and Non-Paris Club creditors.

5. The financial sector remains underdeveloped and plays only a limited role in fostering private sector activity. Following the collapse of Kabul Bank, confidence in the banking sector is low; after an initial fall, deposits have stagnated at about 20 percent of GDP since mid-2011. There has been virtually no new net lending during the past year, reflecting limited credit demand - partly due to low confidence - and banks' difficulties in identifying 
sound lending opportunities. The interest rate on 28-day capital notes remained stable, at around 2 percent.

6. The outlook for 2012 is challenging, but growth should still accelerate. The global and regional economic environment is difficult, with rising oil prices and occasional trade disruptions with neighboring countries. Domestically, the low level of security and the impact of the ongoing military withdrawal will weigh on activity. On the other hand, we expect agriculture to rebound after poor harvests in the last two years. On balance, we project growth to reach about 7 percent in 2012. With some tightening of monetary policy, we aim to contain inflation to $7 \frac{1}{2}$ percent year-on-year by end-2012. Exports are likely to be sluggish, while fuel prices will push up our import bill. We expect that the resulting large trade deficit will continue to be covered by donor support.

\section{Program Implementation Through June 2012}

\section{Against the backdrop of a challenging environment, we have made good} progress on our economic program through June 2012. We have met all but one of the end-March 2012 quantitative performance criteria, and have implemented five out of six envisaged reform steps, though in some cases this took extra time.

\section{A. Quantitative Program}

\section{In the fiscal area, budget implementation improved further, but revenue} collections fell slightly short of expectations. Development spending increased by 22 percent in 2011/12 over the previous year, reaching about 6 percent of GDP. Better capacity in line ministries and more realistic budget projections led to a budget implementation rate of 52 percent. At the same time, as explained in our letter of intent, revenue collection fell short of our target and declined marginally in percent of GDP, reflecting trade disruptions at the border with Pakistan and problems in revenue administration. We restrained operating expenditure in the first half of the fiscal year, and that contributed to containing the operating budget deficit to 6.1 percent of GDP, slightly above our program ceiling.

9. In the monetary area, we continued to build our international reserves buffer, while containing reserve money growth. On the back of strong financial inflows, net international reserves reached US\$5.9 billion at end-March 2012, US\$220 million over the program floor. We sterilized some of these inflows through the capital notes issued by $\mathrm{Da}$ Afghanistan Bank (DAB), and reserve money growth was limited to 16 percent year-on-year in March 2012, compared to 19 percent envisaged under the program.

\section{B. Structural Reforms}

10. We have completed five out of six structural benchmarks for the first review. In December 2011 we submitted the VAT law to the ministry of justice for legislative review- 
we have benefited from Fund's assistance in drafting this law. By January 2012, 25 branches of New Kabul Bank have been closed. In March we completed rolling out the new border management model to two additional border crossing points. In May the minister of finance and the governor of the central bank signed a Memorandum of Understanding on Central Bank's Capitalization, and an internationally reputable external auditor has been appointed for the central bank. Also in May, we prepared amendments to the legislation on state-owned enterprises to the ministry of justice (Fund's comments are now being integrated), and finalized our national strategy to build capacity to respond to economic crime which has been incorporated in the NPP on "Justice for All". Finally, following the approval on February 21, 2012 by the economic committee of the cabinet of the sale of New Kabul Bank, we have appointed a privatization advisor for the bank.

\section{We remain committed to maximizing the recovery of assets from Kabul Bank} shareholders and related parties who benefited from the fraud. Dealing with this decisively, effectively and transparently will not only reduce the enormous fiscal costs of the bail-out, but send a clear message to the public, banks, and future investors that Afghanistan's financial sector is governed and protected by the rule of law-which we know will be crucial to economic growth going forward. The independent Monitoring and Evaluation Commission is conducting an in-depth public inquiry into the Kabul Bank crisis to identify lessons learned (by September 2012; structural benchmark).

\section{Asset recovery has accelerated since the completion of a forensic audit and the} issuance of a presidential decree. A forensic audit completed in March documented the various criminal violations in the bank and determined the exact amounts owed as of August 31, 2010, totaling US\$935 million, of which US\$74 million are from regular borrowers. Following that, a presidential decree dated April 4, 2012 gave beneficiaries until June 4, 2012 to repay their debts, or face criminal and civil action. The decree also called for the establishment of a special tribunal, which was subsequently established by the Supreme Court on April 18, 2012. Based on the guidance from the decree, we have initiated an asset recovery process for each of the beneficiaries (individuals or corporations) identified in the forensic audit report as beneficiaries of the scheme to defraud the bank for the amounts specified therein. Given the, in general, poor payment performance of the existing legally binding agreements, we decided to discontinue this option for shareholders or related parties to repay their obligations to the receivership. Only regular borrowers can continue to repay on their original schedule.

\section{We subsequently ensured that all competent authorities have a common} interpretation of their roles, responsibilities, and legal jurisdictions in asset recovery and the jurisdiction of the special tribunal mentioned in the decree. These include the receiver, the Financial Dispute Resolution Commission, the ministry of justice, and the courts, as well as competent law enforcement authorities and the attorney general's office. The attorney general, the special tribunal, law enforcement (e.g., the Major Crimes Task Force), will concentrate their efforts, inter alia, on building criminal cases, conducting 
investigations that identify crimes and assets owed to the receiver, engaging in international cooperation and investigations, prosecuting criminal behavior and freezing, seizing and confiscating assets. The receiver and the Financial Dispute Resolution Commission will concentrate their efforts on indentifying, taking possession of, and managing recovered assets.

\section{The decree has been implemented and the process has yielded cash and in-kind recoveries, and a number of individuals have been referred to the special tribunal. As of} June 4, 2012, cash recoveries from Kabul Bank beneficiaries amount to US\$128 million out of US\$935 million identified assets transferred to the receiver - an additional US\$54 million since November 2011. The presidential decree expedited the cash recovery process by bringing in US\$30 million since its issuance on April 4, 2012. In addition, we have taken over properties with an estimated value of US $\$ 44$ million in Dubai, and properties with a book value of US\$146 million in Afghanistan. No beneficiary identified in the forensic audit report has paid in full. The two main architects of the fraud have been referred to the special tribunal, and the remaining beneficiaries will be dealt with by the Financial Dispute Resolution Committee, and, if warranted, could be referred to the special tribunal at a later date. In the case of Pamir Airways, Gulbahar Towers, and the Zahira oil tank farm, assets have been transferred to or are under the full legal control of the receiver. In order to assist our investigations and our efforts to recover assets outside of Afghanistan, we have issued mutual legal assistance requests to the United Arab Emirates, and will issue similar requests to other relevant foreign jurisdictions shortly. We will report on the status of asset recovery on a quarterly basis to the public by posting progress on our website - starting in September-including on cash recoveries, taking over of nonfinancial assets, and the status of civil and criminal proceedings.

\section{There has also been significant progress on structural reforms in the fiscal}

sector. First, in line with program budgeting reform, all budgetary units now prepare a single common program-based budget presentation integrating the operating and development budgets. Second, financial plans piloted in the ministry of defense and ministry of interior have been rolled out to nine additional ministries. Third, in accordance with public financial management legislation, the Internal Audit Department of the ministry of finance performs internal audits and similar departments in four additional line ministries have been strengthened. Fourth, in the increasingly important mining sector, we reviewed the consistency between our natural resource and income tax laws and have started the process of amendment; we are developing capacity in mining revenue projections and would welcome technical assistance in the design of an appropriate fiscal regime. Fifth, we continue to improve the medium term fiscal framework-for example by developing a security sector projections model — and are applying it more consistently to assess future fiscal developments. Sixth, we are developing an asset register that will enable us to evaluate more accurately the recurrent costs associated with those assets put in place by past donor investment. Finally, in consultation with the IMF, the new organizational structure for the Afghanistan Customs Department has been approved by the ministry of finance and is now 
awaiting the final approval by the civil service commission. In addition, capacity continues to be bolstered for the conduct of post-clearance audits.

\section{ECONOMIC Program Through June 2013}

16. Our overall economic and development agenda continues to be guided by the ANDS. We continue to aim at poverty reduction, macroeconomic and financial stability, fiscal sustainability, transparency and efficiency of public spending. To this end, we remain committed to the policies agreed under the ECF-supported program, including those spelled out in our previous memorandum from November 1, 2011. Specifically, we will continue to our efforts to enhance economic governance, safeguard and develop the financial system, and move toward fiscal sustainability, including by increasing domestic revenues, and reducing the fiscal risks from public enterprises.

\section{A. Macroeconomic Policies}

\section{Macroeconomic policies aim to provide the fiscal space for development} spending while maintaining a low and stable inflation environment. Given Afghanistan's large development needs and limited domestic resources, we remain highly dependent on donor support to meet our fiscal and external financing needs. The operating balance excluding grants is our fiscal anchor, while reserve money remains the monetary anchor. We will also take measures to support financial stability.

\section{Fiscal policies remain focused on revenue mobilization and strengthening public} financial management. The 2012 budget, approved in April, reflects our reform effort and is consistent with the program (as initially designed for 2012/13). The budget envisages domestic revenue collection to increase to 11 percent of GDP compared to 10.6 percent of GDP during the same period in 2011. Development spending, including on health and education, is targeted to grow by about $1 / 2$ percent of GDP over the last three quarters of 2011 to 6.4 percent of GDP in fiscal year 2012. The budget includes the allocation of US\$65 million for the 2012 amortization of the promissory note issued to DAB for the lender of last resort loan to Kabul Bank. We will also continue with the civil service pay and grade reforms.

\section{Monetary policy will be geared to contain the recent acceleration of headline} inflation and potential second round effects. Based on our estimate for money demand growth, we believe that a reserve money growth of about 19 percent by end-2012 is appropriate. This is somewhat higher than initially envisaged under the program, and is intended to provide some cushion for the anticipated changes in seasonality stemming from the shift in the fiscal year, which is likely to lead to a bunching of government spending in the final quarter of 2012. Net foreign exchange purchases will be the main driver of reserve money growth, and we will use capital notes issuances as needed to fine-tune the course. 


\section{We will maintain a floating exchange rate that will allow the rate to reflect market fundamentals, with interventions aiming at smoothing out volatility and} achieving our reserve money and international reserves targets. We will also adopt measures to deepen the foreign exchange market. We will move towards a system in which foreign currency will be exchanged on an open market, with sales to the central bank as a last recourse, and the central bank using its purchases mainly to steer reserve money growth. To this end, the central bank has been reducing its role in providing currency through foreign exchange auctions by raising minimum bid amounts in these auctions and charging service fees for cash transactions

\section{B. Structural Reforms}

\section{Safeguarding the financial sector and strengthening governance}

\section{We are revising the banking law and will subsequently adapt financial sector} regulations accordingly. The revisions will strengthen provisions on corporate governance, enforcement of capital requirements, large exposures, and related parties, consolidated supervision, early intervention enforcement, and bank resolution. The revisions to the current conventional banking law were finalized in 2011 with support from the IMF, and have undergone review by the Supreme Council. We have also added provisions on Islamic banking, and will submit the revised banking law to parliament by end-September 2012 (structural benchmark). Then, by end-December 2012, we will adapt central bank regulations in the key areas in which the banking law has been revised - corporate governance, capital requirements, related parties, and large exposures - and prepare and issue informational circulars and trainings (structural benchmark). Also, because capacity constraints in the judicial system are some of the main risks to contract enforcement by banks (in particular, loan contracts), financial integrity, and financial development, we intend to create capacity amongst court officials in relevant banking laws and commercial codes, so that the courts can supplement and enhance contract enforcement efforts by banks - to that effect, by endDecember 2012, we will have established a training program for these court officials.

\section{We are also further strengthening banking supervision. We have developed a} work plan to review and strengthen enforcement regulations and the draft enforcement manual, and we are working to improve timeliness for provision of enforcement actionsand hence their impact - through streamlining the examination and special supervision process, as well as monitoring and following up forcefully on the outstanding enforcement orders. We have recently hired 14 additional staff for the supervision department and hope to fill all current vacancies by end-December 2012, although attracting qualified candidates remains challenging. Moreover, we will adopt a new organizational structure for the Financial Supervision Department by end-September 2012 to better utilize existing staff (structural benchmark), and also increase the number of authorized positions from 97 to 137 . Furthermore, we intend to strengthen supervisory staff protection from prosecution for good faith conduct of their duties, including through amendments to the law, where necessary. 
Lastly, the mandate of the Risk Management Department will be modified to focus exclusively on risks within DAB. Staff from that department have been transferred to the Financial Supervision Department and will be trained in risk-based banking supervision.

23. The banking sector remains weak, with 8 out of 12 domestic banks scoring in the lower range of supervisory ratings. ${ }^{1}$ Recognizing that a thorough examination of the sector was merited, we have completed audits of 10 smaller banks with the help of the World Bank. The audits largely confirmed the findings of Financial Supervision Department's on-site examinations: The sector's main vulnerabilities stem from inadequate capital, deficiencies in governance, and excessive exposures. On the basis of these conclusions, the Financial Supervision Department will develop strategies for all structurally weak banks which will be approved by DAB's Supreme Council by end-September 2012. These strategies will envisage that any bank that is unable to increase its minimum paid-up capital to the new level of US $\$ 20$ million by June 2012 will be merged or closed, with the process starting no later than December 2012 (structural benchmark). In parallel, we will strengthen capital adequacy requirements by adapting the risk weights on assets and loan classification and provisioning requirements in line with Basel I standards. Lastly, DAB will restore reserve averaging to its minimum reserve requirement regulation in order to strengthen the incentives and options for banks to more carefully manage their liquidity.

\section{An external audit confirmed the earlier findings of DAB's on-site examination of} a bank with respect to regulatory compliance by the bank, and found no fraud similar to that perpetrated at Kabul Bank. The bank has violated exposure limits, including to a related party, and some years back, the bank appears to have been involved in a misuse of loans, though these loans have been paid back. However, over the past year, the bank has increased capital, addressed violations highlighted by the central bank and improved compliance. We agreed to the bank's request that the additional capital injection planned for March would be delayed until June to allow time to complete good faith negotiations over the sale of some real estate properties. In the meantime, we have started another regular full scope examination of the bank to determine whether additional enforcement actions are necessary. This could include, but is not limited to the injection of additional capital and/or sanctions for unaddressed (or slippages on addressed) violations identifies in DAB's own 2011 examination.

25. New Kabul Bank has been operating according to its business plan. The bank has provided regular prudential reports to the DAB on a monthly basis. Deposits have stabilized at around US\$600 million and expenses have been cut substantially, reducing operating losses. Given our decision that New Kabul Bank should not engage in lending so that it can be presented with a clean bank at the point of sale, the bank is making monthly losses of

\footnotetext{
${ }^{1}$ New Kabul Bank is one of the 12 domestic banks but it has not yet been rated.
} 
around US\$2-3 million. The economic committee of the council of ministers approved the sale of New Kabul Bank on February 21, 2012, and President Karzai endorsed the decision on April 17, 2012. Due to delays in appointing a privatization advisor-the advisor started work only in early May_we will need to move back the benchmark on offering the bank for sale to September 2012 from June (structural benchmark). The economic committee of the cabinet will approve the privatization plan which will be prepared by the privatization advisor by end-August (structural benchmark); the plan includes clear criteria for bidders, precise determination of what is being sold, the procedures for sale, and public communication strategy. Also by end-August, we will finalize the valuation of fixed assets, thus completing the valuation of the bank's balance sheet. We will perform necessary due diligence, including on beneficial ownership of bidders, and only accept reputable, fit-andproper, financially sound investor(s) with expertise in the banking sector and high standard of corporate governance.

\section{If there is no interest from a suitable buyer or the sale fails, we will wind down} New Kabul Bank by end-December 2013. This timeline is longer than we had previously expected because it will take longer than hoped for to hand over the salary payment function to other banks in full as well as migrate the existing deposit accounts to viable financial institutions. Intermediate steps to be taken to prepare New Kabul Bank for liquidation will include, but are not limited to, closure or transfer of credit card business, elimination of interest bearing deposit accounts, transfer of nongovernment salary related deposit accounts elsewhere, and immediate reduction of staff not required for government salary payment administration.

\section{While New Kabul Bank will continue to play a leading role in effecting salary} payments for government employees, other banks are slowly taking over some of this function. Over 400,000 government employees now receive their salaries by direct deposit to their bank accounts (largely at New Kabul Bank) or third party intermediaries. In June we awarded the new salary payment contract for the next three years to several banks that have participated in the bidding process. New national payments systems (real time gross settlement, automated clearing house, and a national switch) are expected to be operational by mid-2014. At that time, government employees will be able to select the bank in which their salaries are paid, thus introducing genuine market competition for provision of the service.

\section{Afghanistan's financial sector is nascent and the criminal justice and regulatory} and institutional framework that support financial and economic activity lack capacity and experience. These weaknesses are among the key factors that are slowing asset recovery in the case of Kabul Bank. We recognize the extreme threats and vulnerabilities which threaten economic stability, including an overwhelmingly large illicit narcotics and smuggling sector, a large cash based economy, a large amount of unregulated financial activity taking place outside the formal regulated sector, high levels of corruption, powerful 
vested interests, porous borders which impede revenue collection, and vast areas of the country that cannot fall under financial supervision due to insecurity.

\section{As such, our economic crimes strategy aims to build capacity and foster} cooperation among relevant government entities to enable them to respond to these

risks. To this end, we will establish a senior official-led interagency steering committee-the Economics Crime Task Force-(structural benchmark) to oversee the implementation of the new banking law, the AML and terrorist financing laws and their provisions, and to function as a coordinating body for the implementation of the economic crimes strategy and its action items. The committee will be responsible for: (i) ensuring and coordinating the education of the competent authorities on the new provisions to the laws related to economic crimes (especially, but not exclusive to) the banking law, the AML law and the CFT law); (ii) ensuring there is a commensurate issuance of necessary regulations, circulars and trainings within agencies and the private sector following the passage of the laws; (iii) increasing the capacity of the justice system to investigate, try, and hear financial crimes and related money laundering, terrorist finance and asset recovery cases/procedures; (iv) the establishment of a consolidated handbook of guidelines and procedures for investigating and trying economic crimes according to Afghan law that contains definitions of the roles and responsibilities of competent authorities including courts, the attorney general's office, the central bank, the financial intelligence unit, the Financial Disputes Resolution Committee, and the Major Crimes Task Force; and (v) monitoring the implementation of appropriate programs for competent authorities on new and existing institutional procedures, legal provisions, and regulations.

\section{We are also taking urgent actions to address deficiencies in our AML/CFT}

framework. Following its first AML/CFT mutual evaluation, Afghanistan was found to be deficient in a number of areas. As part of the International Cooperation Review Group's targeted review process we have made a ministerial level commitment to an action plan to be agreed with the Financial Action Task Force in June 2012, key elements of which will be implemented by September 2012 (structural benchmark). In particular, we will submit to parliament by end-September 2012 our new AML and CFT laws. Finally, by June 2013, the AML/CFT stakeholders, including the Financial Transactions and Reports Analysis Center of Afghanistan, DAB's Financial Supervision Department, and competent law enforcement authorities, will agree in a memorandum of understanding on a mandatory exchange of AML/CFT information and analyses (structural benchmark). This memorandum will mandate the exchange of (i) analytical information - specifically in the form of biennial reports for all AML/CFT stakeholders by the Financial Transactions and Reports Analysis Center of Afghanistan identifying risks, threats, and typologies; (ii) as well as dissemination of tactical analysis products to competent law enforcement authorities in a timely fashion; (iii) dissemination of information on reporting trends and suspected instances of noncompliance (for DAB AML/CFT supervisors); and (iv) the production of annual statistical reports on the exchange of information. 


\section{Strengthening the central bank capitalization framework and governance}

31. The memorandum of understanding between DAB and the ministry of finance sets out a path toward a strengthened central bank capitalization framework. This memorandum, inter alia, reconfirms that the authorized capital be five percent of the sum of currency in circulation, debt securities issued by DAB and the credit balances of commercial bank's accounts maintained on the books of DAB, and aligns the provisions on the netting and allocation of net income with international good practice. Any required recapitalization will be effected no later than 2014. According to the memorandum, we will draft the necessary amendments to the central bank law (in consultation with the Fund) and submit them to the ministry of justice by December 2012, with the view to submitting them to parliament by June 2013 (structural benchmark).

\section{The central bank is committed to ensuring timely implementation of the} recommendations of the update safeguards assessment. In this context, we will strengthen the internal audit function (with external assistance) and enhance the Audit Committee's oversight as a priority. We have recently appointed the head of the Audit Committee and intend to fill the two vacant posts by August 2012.

\section{Moving toward fiscal sustainability while providing space for development spending}

33. Our fiscal strategy focuses on revenue generation and minimizing fiscal risks to ensure that the necessary resources are available for development spending. Revenue generation efforts include the introduction of a VAT and designing and legislating the fiscal regime for the natural resources sector, and continued reforms in customs and tax administration. ${ }^{2}$ We are also taking steps to improve budget planning and implementation and to contain the fiscal risk stemming from state-owned enterprises and corporations. To improve cash management, we will introduce sukuks as government securities in 2014.

34. The VAT law is under preparation. The law is currently being reviewed by the ministry of justice. It will be sent to cabinet for discussion in September 2012 and to parliament for approval by end-December 2012 (structural benchmark). As we finalize the draft, we will ensure that exemptions are limited to medical, education, and financial services as well as humanitarian and religious purposes, and are explicitly listed in the VAT law. We will also revise the current draft to require that any future exemptions are provided for through an amendment of the VAT law that is approved by parliament.

\footnotetext{
${ }^{2}$ The pilot border management model at Hairatan will be applied fully to the Hairatan railway border crossing. The Afghan Border Police and the Afghanistan Customs Department will take full control of the railway border crossing by end-September 2012, and the ministry of commerce and industries will at most provide logistical services, but no longer be in charge of operating any part of the facility.
} 
35. Planning for the VAT implementation has begun. Given the VAT threshold, the tax will initially be collected only from large taxpayers registered with the large taxpayer office (LTO). Successful VAT introduction, therefore, hinges upon the identification and registration of all taxpayers whose turnover exceeds the Large Taxpayer Office (LTO) threshold. Currently, many large taxpayers (importers) pay a fixed rate (a presumptive tax) in order to avoid filing, thereby remaining outside the LTO or outside the formal tax net altogether. Therefore, by end-December 2012 and consistent with the recent Fund advice on VAT implementation, The Afghanistan Revenue Administration will identify taxpayers in the Medium Taxpayer Office (MTO) and the Small Taxpayer Office (STO) whose turnover exceeds the LTO threshold, revisit the thresholds for the large taxpayer office and the medium taxpayer office with a view to having an efficient allocation of taxpayers across the three offices - no more than 350 taxpayers in the LTO — and transfer cases into the LTO accordingly (structural benchmark) in time for the submission of the VAT law to parliament. The findings and follow-up actions will be summarized in a report. The above will be complemented with significant increases in the number of staff as well as training, which will enable the LTO to have the adequate capacity to administer all taxpayers. In order to ensure the stability of the VAT taxpayer base and to level the playing field across businesses, we will also immediately discontinue all "concessions" for importers ("traders"). ${ }^{3}$ This is an important commitment, as initially a large proportion of VAT revenues will be collected at the border. Therefore, eliminating concessions for certain taxpayer groups will prevent continued undermining of the revenue base in light of our weak revenue performance in 2011/12.

\section{Our natural resources are an important part of our economic future and we} understand that a number of policy issues must be addressed to harness this wealth for the benefit of the population. The development of our natural resource wealth, including the rate of exploitation, will be integrated into the country's long-term development program. Specifically, all revenues from extractive industries will be channeled transparently through the budgetary process where national spending priorities are set. There will be no use of earmarking or off-budget spending. We will consult closely with the World Bank and the IMF in designing the framework for managing our natural resources.

\section{We will put in place a sound and robust fiscal regime for the mining sector to} attract investment and ensure the government has a reasonable share of the economic rent. Over the past few years we have benefited from numerous studies, including by the

\footnotetext{
${ }^{3}$ Namely, in fiscal year 2012, we have not renewed or granted any concessions and will revoke any existing concessions by end-June 2012, effectively obliging the beneficiaries to file and pay taxes as otherwise required. We will enforce this obligation on the beneficiaries in case they fail to comply.
} 
Fund, reviewing the current framework and recommending changes to legislation. ${ }^{4}$ In that regard, by end-June 2013, we will submit to the ministry of justice amendments to the appropriate laws that create an effective and transparent fiscal regime for natural resources in full compliance with the Extractive Industries Transparency Initiative and in consultation with the IMF (structural benchmark).

\section{To strengthen budget planning and implementation, we are adopting program} budgeting. As part of the preparation for migration to program-based appropriations, by endMarch 2013, we will conduct a thorough review of preparedness for migration, including, inter alia, verifying under the program structure the alignment of policies with budgetary allocations, consistency in program definitions across agencies, linkages between the program and agencies' organizational structures, clear rules for program level appropriation control (and other levels of expenditure control) during budget execution, clear rules for reallocation between line/economic items within each program (e.g., putting restrictions on certain line items such as salaries to prevent abuse), and a detailed appraisal of areas with capacity and accountability shortfalls of program managers to execute their budgets.

\section{We are working on the introduction of marketable sukuk securities to improve} cash management with assistance from the IMF. We have finalized an initial assessment of laws for their suitability for Islamic finance, and now are finalizing the draft sukuk law, with a view to submitting it to the ministry of justice by September 2012 (structural benchmark) and to the parliament by March 2013. ${ }^{5}$ This is a delay compared to our earlier plans because it will take longer to ensure that the draft law is consistent with the Afghan legal tradition and we want to consult widely with all stakeholders on the law. We continue to aim for the first issuance in late 2013 or early 2014. In this respect, we will produce a sukuk implementation plan, with specific actions and a timetable by June 2012, finalize the identification of state-owned assets and development projects that would back sukuks, and set up a sukuk shariah supervisory board by September 2012.

\section{We are taking steps to identify the potential fiscal risks from public} enterprises - state-owned enterprises and state-owned corporations. The fiscal burden of these companies ranges from an immediate budgetary impact due to tax concessions or subsidies, to fiscal risks such as debt guarantees and implicit obligations in the event of failure. We have recently prepared amendments to the legislation on SOEs to bring them

\footnotetext{
${ }^{4}$ See, for example, Afghanistan: Mining Tax and Royalties Policy, USAID Economic Growth and Governance Initiative, 2010; Tax Reform: The Next Steps, International Monetary Fund, 2010; A Review of the Consistency between Afghanistan's Tax and Mining Laws, Adam Smith International, 2011.

${ }^{5}$ Inter alia, we have requested a legal opinion whether debt service (principal and interest) requires annual parliamentary approval, and, if so, we will assess whether an alternative mechanism would be desirable, such as automatic debt service authorization or a debt ceiling. In this respect, we will consult the Fund and prepare a legal and economic assessment of the framework for debt servicing.
} 
under ministry of finance oversight and, after incorporating Fund's comments, will submit the draft to the ministry of justice. We are also working on amendments to the company law that will improve the financial oversight of state-owned corporations (SOCs) by the ministry of finance. We intend to send the amendments to the ministry of justice for review by endDecember 2012 (structural benchmark). By 2014, we will include a fiscal risk statement related to condition of public enterprises and corporations into the published budget documents. The objective is to provide a transparent assessment of the fiscal risks involved in these companies. The state-owned enterprises department will be responsible for preparing this assessment - initially for those enterprises of which the ministry of finance is the main shareholder or owner - as part of the budget preparation process. ${ }^{6}$ As an intermediate step, we will review the financial statements and plans provided by public enterprises to the ministry of finance and issue guidance on what information these statements and plans have to contain by December 2012.

\section{Program Modalities ANd Monitoring}

\section{In 2012, we are changing the fiscal year to facilitate budget planning and} implementation. Through 2011/12, the fiscal year corresponded to the Afghan calendar year (based on the solar year) running from March 21 to March 20 in most years. In 2012 (ministry of finance) and 2013 (central bank), we are transitioning to a fiscal year that is closer aligned with the Gregorian calendar year and runs December from 22 to December 21 in most years. The interim fiscal year 2012 has only 9 months. We request to adjust the schedule of reviews under the program to accommodate this change. Accordingly, the quantitative performance criteria and structural benchmarks are set for September 2012, December 2012, and June 2013; with indicative targets for March 2013 (Tables 1-3 of the letter of intent). The second, third and fourth reviews of the program could be completed before the end of the months of December 2012, March 2013 and September 2013 respectively. We will continue monitoring our reform agenda through our Technical Coordination Committee.

42. Article VIII. During the program period, we will not impose or intensify restrictions on the making of payments and transfers for current international transactions, or introduce or modify multiple currency practices, or conclude bilateral payments agreements inconsistent with Article VIII, or impose or intensify restrictions for balance of payments purposes.

\footnotetext{
${ }^{6}$ At present eight SOCs, which represent 90 percent of total SOEs/SOCs assets, have the ministry of finance as their main shareholder.
} 


\section{AtTaChment II. TeChnical Memorandum of Understanding}

1. This memorandum reflects understandings between the Afghan authorities and Fund staff in relation to the monitoring of the ECF-supported program during 2012-13. It specifies valuation for monitoring quantitative targets under the program (Section I), quantitative performance criteria and indicative targets (Section II), adjustors (Section III), and data reporting (Section IV).

\section{Program Exchange Rates and Gold Valuation}

2. Program exchange rates are used for formulating and monitoring quantitative targets. All foreign assets and liabilities denominated in U.S. dollars will be converted into Afghanis at a program exchange rate of 45.3740 Afghanis per U.S. dollar, which corresponds to the cash rate of March 20, 2011. Gold holdings will be valued at US\$1,418.90 per troy ounce, the price as of March 20, 2011. Assets and liabilities denominated in SDRs and in foreign currencies other than the U.S. dollar will be converted into U.S. dollars at their respective exchange rates of March 20, 2011, as reported in the following table.

\section{Exchange Rate}

U.S. dollars / Canadian dollar
U.S. dollars / U.A.E. dirham
U.S. dollars / Egyptian pound
U.S. dollars / euro
U.S. dollars / Hong Kong dollar
U.S. dollars / Indian rupee
U.S. dollars / Pakistani rupee
U.S. dollars / Polish zloty
U.S. dollars / Iranian rial
U.S. dollars / Saudi Arabian riyal
U.S. dollars / Russian ruble
U.S. dollars / Swiss franc
U.S. dollars / pounds sterling
U.S. dollars / SDR

\section{Program Rate}

1.016000

0.272300

0.168600

1.418200

0.128200

0.022160

0.011715

0.348800

0.000097

0.266600

0.035180

1.109800

1.623400

1.585700

\section{Quantitative Performance Criteria ANd Indicative Targets}

\section{The quantitative performance criteria for September and December 2012, and} June 2013, specified in Table 2 of the letter of intent, are:

- Floors on revenue of the central government and net international reserves (NIR); and

- $\quad$ Ceilings on reserve money; net central bank credit to the central government (NCG); contracting and/or guaranteeing new medium- and long-term nonconcessional external debt by the public sector, (continuous); short-term external debt owed or guaranteed by the public sector (continuous); accumulation of external payment arrears, excluding interest on preexisting arrears (continuous); lending from state- 
owned banks or the central bank to, or government guaranteed borrowing by, public enterprises in need of restructuring (continuous).

4. The above variables also constitute indicative targets for March 2013. In addition, the program includes the following indicative targets for the four above-mentioned dates:

- $\quad$ Ceilings on currency in circulation and on the operating budget deficit of the central government excluding grants; and

- $\quad$ Floor on social and other priority spending.

5. The central government consists of the Office of the President, the ministries and other state administrations and governmental agencies, including the attorney general's office; the National Assembly; and the judiciary, including the Supreme Court.

6. Reserve money is defined as currency in circulation plus Afghani-denominated commercial bank deposits at the central bank, excluding deposits held at the DAB's branches (because of the unavailability of reliable and timely data from the DAB's branches), but including balances maintained by the commercial banks in the DAB's overnight facility.

7. Currency in circulation is defined as total currency issued by the DAB. It excludes currency held in the presidential palace vault, in the DAB main vault, and in the vaults of all provincial and district branches of the DAB.

\section{Rerouting of treasury's IMF accounts to central bank's balance sheet. For} program purposes, the government's financial positions arising from dealing with the IMF is treated as if these functions were performed by DAB on behalf of the treasury, that is as if DAB have assumed these positions and have established corresponding counterpositions visà-vis the treasury.

9. Net central bank credit to the government is defined as the difference between the central bank's claims on the government and government deposits at the DAB, excluding deposits held at the DAB's branches. Claims include the so-called "promissory note," in the amount outstanding (at face value) of DAB's claim on a bank in liquidation that has been guaranteed by the ministry of finance though issuance of a promissory note, and up to the amount specified therein.

10. Net international reserves are defined as reserve assets minus reserve liabilities of the DAB, both of which are expressed in U.S. dollars.

- $\quad$ Reserve assets of the $\mathrm{DAB}$, as defined in the fifth edition of the Balance of Payments Manual (BPM5), are claims on nonresidents denominated in foreign convertible currencies controlled by $\mathrm{DAB}$, and are readily and unconditionally available for DAB 
to meet balance of payments financing needs, intervention in exchange markets, and other purposes. They include DAB holdings of monetary gold, SDRs, Afghanistan's reserve position in the IMF, foreign currency cash (including foreign exchange banknotes in the vaults of the DAB, but excluding cash held in the DAB's branches), and deposits abroad (including balances on accounts maintained with overseas correspondent banks). Excluded from reserve assets are any assets that are pledged, collateralized, or otherwise encumbered; claims on residents; precious metals other than monetary gold; assets in nonconvertible currencies; illiquid assets; and claims on foreign exchange arising from derivatives in foreign currencies vis-à-vis domestic currency (such as futures, forwards, swaps, and options).

- $\quad$ Reserve liabilities are defined as short-term (original maturity) foreign exchange liabilities of DAB to nonresidents (held at DAB headquarters); all credit outstanding from the IMF; foreign currency reserves of commercial banks held at DAB headquarters; commitments to sell foreign exchange arising from derivatives (such as futures, forwards, swaps, and options); and all arrears on principal or interest payments to commercial banks, suppliers, or official export credit agencies.

11. Revenues of the central government are defined in line with the Government Financial Statistics Manual (GFSM 2001) but on a cash accounting basis, excluding grants. Revenue is an increase in net worth of the central government (including its units in the provinces and agencies) resulting from a transaction.

- $\quad$ Revenues of the central government include taxes and other compulsory transfers imposed by central government units, property income derived from the ownership of assets, sales of goods and services, social contributions, interest, fines, penalties and forfeits and voluntary transfers received from nongovernment other than grants. The definition for program monitoring excludes grants and other noncompulsory contributions received from foreign governments and international organizations; such transfers between central government units would be eliminated in the consolidation of the fiscal reports and not recorded as revenue. Receipts collected by central government on behalf of noncentral government units should not be counted as revenue (e.g., Red Crescent fees). Receipts from the sale of nonfinancial assets (e.g., privatization and signature bonuses from natural resource contracts), and transactions in financial assets and liabilities, such as borrowing but excepting interest payments, are also excluded from the definition of revenue.

- $\quad$ Revenues should be recognized on a cash basis and flows should be recorded when cash is received. The official Afghanistan Government Financial Management Information System (AFMIS) reports will be used as the basis for program monitoring. Exceptional advanced payments will be treated as if received on the normal due date. All revenue must be supported by the relevant documentation and revenue receivables, where a cash sum has been recorded but the revenue item has not yet been accounted 
for, and revenues payable, where the revenue has been reported but the cash has yet to be recorded should be separately reported on a gross basis.

12. Social and other priority spending is defined as the sum of pro-poor spending identified in accordance with the ANDS poverty profile by the Ministry of Education, Ministry of Public Health, and Ministry of Labor, Social Affairs, Martyrs, and Disabled within the central government's operating budget for a particular fiscal year.

13. For program purposes, the definition of external debt is set out in Executive Board Decision No. 6230-(79/140), as revised on August 31, 2009 (Decision No. 14416-(09/91).

(a) The term "debt" will be understood to mean a current (i.e., not contingent) liability, created under a contractual arrangement through the provision of value in the form of assets (including currency) or services, and which requires the obligor to make one or more payments in the form of assets (including currency) or services, at some future point(s) in time; these payments will discharge the principal and/or interest liabilities incurred under the contract. Debts can take a number of forms, the primary ones being as follows:

(i) loans (i.e., advances of money) to the obligor by the lender made on the basis of an undertaking that the obligor will repay the funds in the future (including deposits, bonds, debentures, commercial loans and buyers' credits) and temporary exchanges of assets that are equivalent to fully collateralized loans under which the obligor is required to repay the funds, and usually pay interest, by repurchasing the collateral from the buyer in the future (such as repurchase agreements and official swap arrangements);

(ii) suppliers' credits (i.e., contracts where the supplier permits the obligor to defer payments until sometime after the date on which the goods are delivered or services are provided); and

(iii) leases (i.e., arrangements under which property is provided which the lessee has the right to use for one or more specified period(s) of time that are usually shorter than the total expected service life of the property), while the lessor retains the title to the property. For the purpose of the guideline, the debt is the present value (at the inception of the lease) of all lease payments expected to be made during the period of the agreement excluding those payments that cover the operation, repair or maintenance of the property.

(b) Under the definition of debt set out in paragraph 13 (a) above, arrears, penalties, and judicially awarded damages arising from the failure to make payment under a contractual obligation that constitutes debt are debt. Failure to make payment on an obligation that is not considered debt under this definition (e.g., payment on delivery) will not give rise to debt. 
14. Long term and medium term external debt. A ceiling applies, on a continuous basis, to the contracting and guaranteeing by the public sector of new nonconcessional borrowing debt with nonresidents with original maturities of one year or more. The ceiling applies to debt and commitments contracted or guaranteed for which value has not yet been received. This applies to private debt for which official guarantees have been extended and which, therefore, constitute a contingent liability of the public sector. Excluded from the limits are refinancing credits and rescheduling operations, credits extended by the IMF, and credits on concessional terms as defined below. Consistent with the Public Finance and Expenditure Management (PFEM) Law, the MOF should have sole responsibility for the contracting and guaranteeing of external debt on behalf of the government.

- The definition of "government" includes the central government (including government departments), as well as official agencies that do not seek profit and whose budgets are issued independent of the annual operational or development budgets. The public sector comprises the government as defined above, the DAB, and nonfinancial public enterprises. Public enterprises are defined below in paragraph $17 .{ }^{1}$

- $\quad$ For program purposes, the guarantee of a debt arises from any explicit legal obligation of the public sector to service a debt in the event of nonpayment by the debtor (involving payments in cash or in kind), or from any implicit legal or contractual obligation of the public sector to finance partially or in full any a shortfall incurred by the debtor.

- $\quad$ For program purposes, a debt is concessional if it includes a grant element of at least 60 percent, calculated as follows: the grant element of a debt is the difference between the present value (PV) of debt and its nominal value, expressed as a percentage of the nominal value of the debt. The PV of debt at the time of its contracting is calculated by discounting the future stream of payments of debt service due on this debt. ${ }^{2}$ The discount rates used for this purpose are the currency specific commercial interest reference rates (CIRRs), published by the Organization for Economic Cooperation and Development (OECD). For debt with a maturity of at least 15 years, the ten-year-average CIRR will be used to calculate the PV of debt and, hence, its grant element. For debt with a maturity of less than 15 years, the six-month average CIRR will be used. To both the ten-year and

\footnotetext{
${ }^{1}$ Government control of an entity can be established based on the following criteria: (i) ownership of the majority of the voting interest; (ii) control of the board or other governing body; (iii) control of the appointment and removal of key personnel; (iv) control of key committees of the entity; (v) golden shares and options; (vi) regulation and control; (vii) control by a dominant public sector customer or group of public sector customers; and (viii) control attached to borrowing from the government.

${ }^{2}$ The calculation of concessionality will take into account all aspects of the loan agreement, including maturity, grace period, payment schedule, upfront commissions, and management fees.
} 
six-month averages, the same margins for differing repayment periods as those used by the OECD need to be added $(0.75$ percent for repayment periods of less than 15 years, 1 percent for 15 to 19 years, 1.15 percent for 20 to 29 years, and 1.25 percent for 30 years or more).

15. The zero ceiling on short-term external debt applies on a continuous basis to the stock of short-term external debt owed or guaranteed by the public sector (as defined in paragraph 14 of this memorandum), with an original maturity of up to and including one year.

- It applies to debt as defined in paragraph 13 of this memorandum.

- $\quad$ Excluded from the limit are rescheduling operations (including the deferral of interest on commercial debt) and normal import-related credits.

- Debt falling within the limit shall be valued in U.S. dollars at the exchange rate prevailing at the time the contract or guarantee becomes effective.

16. A continuous performance criterion applies to the nonaccumulation of new external payments arrears on external debt contracted or guaranteed by the central government or the DAB. External payment arrears consist of external debt service obligations (principal and interest) falling due after March 20, 2012 and that have not been paid at the time they are due, as specified in the contractual agreements. Excluded from the prohibition on the accumulation of new arrears are (i) arrears arising from interest on the stock of arrears outstanding as of March 20, 2012; and (ii) external arrears that are subject to debt rescheduling agreements or negotiations.

17. Lending to, or guaranteeing borrowing by, public enterprises. The zero ceiling on new lending from state-owned banks or the central bank to, or government guaranteed domestic borrowing by, public enterprises in need of restructuring applies on a continuous basis.

- $\quad$ For the purposes of this performance criterion (i) "state-owned banks" refers to those banks that are wholly or majority owned by the government (as defined in paragraph 14 of this memorandum), including Bank Millie, Bank Pashtany and New Kabul Bank; (ii) "public enterprises in need of restructuring" refers to enterprises that meet either of the following: (a) public enterprises that have not had an audited balance sheet in the past two fiscal years, (b) public enterprises that have been identified by the Ministry of Finance for liquidation, or (c) public enterprises that do not have cabinetapproved restructuring plans; and (iii) "public enterprises" refers to enterprises wholly or majority owned by the government, including those covered by the State-Owned Enterprise (Tassady) Law, and all state-owned corporations and any other public entities and government agencies engaged in commercial activities but not covered by the Tassady Law. 
- It applies to any new loans (or financial contributions) extended directly from the central bank or state-owned banks to public enterprises in need of restructuring, and also to any explicit government guarantees for borrowing undertaken by these public enterprises (including loan agreements and guarantees for which value has not been received).

18. Operating budget deficit of the central government excluding grants is defined as revenues of the central government minus operating budget expenditure recorded in AFMIS.

\section{AdJUSTORS}

19. The floor on NIR and the ceiling on the NCG are consistent with the assumption that core budget development spending will amount, on a cumulative basis from the beginning of the respective fiscal year, to:

$\begin{array}{ll}\text { September 21, 2012 } & \text { Af 23.9 billion } \\ \text { December 20, 2012 } & \text { Af } 49.6 \text { billion } \\ \text { March 20, 2013 } & \text { Af } 15.9 \text { billion } \\ \text { June 21, 2013 } & \text { Af } 33.7 \text { billion }\end{array}$

Should core budget development spending exceed these projections, the NIR floor will be adjusted downward and the NCG ceiling will be adjusted upward by the difference between the actual level (up to the appropriated amount) and the projected level of development spending.

20. The NIR floor and NCG ceiling are defined consistent with the assumption that the external financing of the core budget and receipts from the sale of nonfinancial assets will amount, on a cumulative basis from the beginning of the respective fiscal year, to:

$\begin{array}{ll}\text { September 21, 2012 } & \text { Af } 58.7 \text { billion } \\ \text { December 20, 2012 } & \text { Af } 96.2 \text { billion } \\ \text { March 20, 2013 } & \text { Af } 33.8 \text { billion } \\ \text { June 21, 2013 } & \text { Af } 67.8 \text { billion }\end{array}$

Should external financing of the core budget (including that associated with off-budgetary spending coming on budget) and the receipts from the sale of nonfinancial assets collectively exceed (fall short of) these projections, the NIR floor will be adjusted upward (downward) and the NCG ceiling will be adjusted downward (upward) by the difference between their actual level and the projected level. The overall downward adjustment to the NIR floor will be capped at US $\$ 500$ million and the overall upward adjustment to the NCG ceiling will be capped at Af 25 billion.

21. The NIR floor and NCG ceiling are defined consistent with the assumption that the asset recovery from banking sector institutions in liquidation applied towards DAB's claim on the 
ministry of finance will amount, on a cumulative basis from the beginning of the respective fiscal year, to:

$\begin{array}{ll}\text { September 21, 2012 } & \text { Af } 2.5 \text { billion } \\ \text { December 20, 2012 } & \text { Af } 3.8 \text { billion } \\ \text { March 20, 2013 } & \text { Af } 0.6 \text { billion } \\ \text { June 21, 2013 } & \text { Af } 1.3 \text { billion }\end{array}$

Should such asset recovery exceed (fall short of) these projections, the NIR floor will be adjusted upward (downward) and the NCG ceiling will be adjusted downward (upward) by the difference between the actual recovery and its projected level.

22. Should the ministry of finance recapitalize (receive dividend from) DAB, the NCG ceiling will be adjusted upward (downward) by the amount of this recapitalization (dividend).

23. Should Afghanistan receive an SDR allocation the NIR floor will be adjusted upwards by the amount of this allocation.

\section{Should some expenditure currently financed directly by donors outside the} budget be moved on to the operating budget, the NIR floor will be adjusted downward, and the NCG ceiling and the indicative targets (ceilings) for the operating budget deficits of the central government, excluding grants, will be adjusted upward, by the actual amount of these expenditures on the conditions that (i) the moving on budget of these expenditures is justified by a statement from donors indicating their decision to stop financing them outside the budget; and (ii) they are subject to a supplementary appropriation approved by parliament. The overall downward adjustment to the NIR floors will be capped at US\$300 million.

\section{Provision OF Information to the Fund}

25. To facilitate monitoring of program implementation, the government of Afghanistan will provide the Fund through the office of the Resident Representative of the IMF in Afghanistan, the information specified below and summarized in the list of reporting tables provided to the Technical Coordination Committee.

26. Actual outcomes will be provided with the frequencies and lags indicated below.

- DAB net international reserves: weekly, no later than two weeks after the end of each week.

- Monetary statistics, including exchange rates, government accounts with the DAB, currency in circulation, reserve money, and a monetary survey: monthly and no later than three weeks after the end of the month (six weeks in the case of the monetary survey). The monetary survey will include the balance sheet of the DAB and a consolidated balance sheet of the commercial banking sector. 
- $\quad$ Core budget operations and their financing: monthly and no later than four weeks after the end of the month. The official reports for program monitoring will be the monthly financial statements from the Afghanistan Financial Management Information System. The structure of financing (grants and loans should be separately identified) and expenditure data should be on a consistent cash basis. Core operating expenditures should be reported on a monthly basis using the budget appropriation economic and administrative classification in addition to the program and functional classification as reported in the budget documents. Core development expenditures should also be reported separately on a monthly basis using the budget program classification in addition to the economic, administrative and functional classification consistent with the operating budget. All the data should also compare outturns against the approved budget. Core operating and development revenues and expenditures should also be reported by province, separately on the same monthly basis.

- $\quad$ External budget operations and their financing (i.e., donor funded spending outside the core budget treasury systems): semiannually (more frequently if possible) and no later than eight weeks after the end of the period. External development expenditures should be reported on a disbursement basis (as currently defined in budget documents) using the budget program classification (and an administrative, functional and provincial classification where possible).

- External debt data: quarterly and no later than six weeks after the end of the quarter. These will include: (i) details of new loans contracted or guaranteed during the quarter, including the terms of each new loan; (ii) the stock of debt at the end the quarter, including short-term debt, and medium- and long-term debt; (iii) loan disbursements and debt service payments (interest and amortization) during the quarter; (iv) debt relief received during the quarter; (v) information on all overdue payments on short-term debt, and on medium- and long-term debt, including new external arrears (if any); and (vi) total outstanding amount of arrears.

- National accounts data: annually and no later than three months after the end of the year. Merchandise trade data should be reported quarterly and no later than eight weeks after the end of the quarter.

- Monthly consumer price indexes (CPIs) for Kabul and other major cities ("national" CPI) with a lag of four weeks after the end of each month.

- $\quad$ Four-monthly and with a three-month lag, financial flows and other key variables of the state electricity company (DABS), aggregated as well as disaggregated by regional hubs, for the preceding 12 months and (in the last four-monthly period of the year) a forecast for the following fiscal year. The report will use the template agreed with Fund staff, with all lines filled in, but excluding the disaggregation of loses into 
technical and nontechnical for regions outside Kabul, which is expected by March 20, 2013.

- $\quad$ Financial Stability Indicators for each commercial bank: quarterly and with a onemonth lag after the end of each quarter. These indicators will include banks' prudential ratios, capital adequacy ratio, liquidity ratio, portfolio quality indicators (e.g., nonperforming loans, provisions as percentage of classified loans), concentration ratio, related loan ratio, information on open foreign exchange positions, large loan and deposits statistics, income and expense information such as net income to total assets ratio (ROA), rate of return on capital, and net interest margin, and other relevant information. Monthly, balance sheet and income statement for each bank compiled from supervisory submissions, as well as the Summary Analysis of Condition and Performance of the Banking System.

- $\quad$ Lending to public enterprises from each commercial bank: quarterly with a one-month lag after the end of each quarter share a report on the following balance sheet items and operations for each bank: (i) aggregate value of outstanding loans to all public enterprises; (ii) disaggregated value of outstanding loans by public enterprise for each bank's top 10 borrowers; and (iii) indicators of the quality of these loans. For this reporting requirement, public enterprises refer to those defined in point (iii) in the first bullet of paragraph 17 .

- Monthly activity and cost reports from the Kabul Bank receiver, including the status and financial details of asset recovery.

- $\quad$ Monthly detailed balance sheet and income statement for New Kabul Bank (with a two weeks lag) as well as quarterly reports on bank's progress against its business and financial plans (staffing, branches, etc).

- Quarterly, transactions on the Kabul Bank loan account, Kabul Bank receivership accounts and any other accounts related to the bank or asset recovery from the bank.

- $\quad$ Copies of documents related to asset recovery, such as mutual legal assistance requests (evidence and banking and property information redacted) to foreign jurisdictions, copies of MOUs which cover international cooperation with said jurisdictions.

- Monthly details of the discretionary cash balances held in the Ministry's AFS and U.S. dollar-denominated TSA accounts, and the discretionary development 27232 account. In addition, an update of the monthly summary report of funds under operating budget, summaries of expenditure for both the operating budget and discretionary development budgets, and the updated cash projections for the current and following fiscal years. 
- Copies of documents related to the progress on structural benchmarks under the program, such as draft legislation, memoranda of understanding, strategies, implementation plans, transmittal letters, etc.

27. The Technical Coordination Committee (TCC) will send to the IMF reports by the end of each quarter documenting progress in implementing structural benchmarks under the program. These reports will include appropriate documentation and explain any deviations relative to the initial reform timetable, specifying expected revised completion date. Other details on major economic and social measures taken by the government that are expected to have an impact on program sequencing (such as changes in legislation, regulations, or any other pertinent document) will be sent in a timely manner to IMF staff for consultation or information. 


\title{
INTERNATIONAL MONETARY FUND
}

ISLAMIC REPUBLIC OF AFGHANISTAN

First Review Under the Extended Credit Facility Arrangement, Request for Waiver of Nonobservance of a Performance Criterion, Modification of Performance Criteria, and Rephasing of Disbursements

\author{
Informational Annex \\ Prepared by the Middle East and Central Asia Department
}

June 19, 2012

Contents

Page

Annexes

I. Islamic Republic of Afghanistan — Relations with the Fund ...........................................

II. Islamic Republic of Afghanistan - Relations with the World Bank and Bank-Fund Collaboration Under the Joint Management Action Plan ....................................................

III. Islamic Republic of Afghanistan—Relations with the Asian Development Bank .............11 
ANNEX I. ISLAMIC REPUblic OF AfGHANISTAN-RELATIONS WITH THE FUND

(As of May 31, 2012)

I. Membership Status: Joined July 14, 1955; Article XIV.

II. General Resources Account

Quota

Fund holdings of currency

Reserve position in Fund

III. SDR Department

Net cumulative allocation

Holdings

IV. Outstanding Purchases and Loans

ECF Arrangements \begin{tabular}{rr} 
SDR Million & \% Quota \\
\hline 161.90 & 100.00 \\
161.92 & 100.01 \\
0.00 & 0.00
\end{tabular}

$\frac{\text { SDR Million }}{155.31}$

128.25

$\underline{\text { SDR Million }}$

87.35 $\underline{\% \text { Allocation }}$ 100.00

82.57

\% Quota

53.95

V. Latest Financial Arrangements:

\begin{tabular}{|c|c|c|c|c|}
\hline Type & $\begin{array}{l}\text { Date of } \\
\text { Arrangement }\end{array}$ & $\begin{array}{l}\text { Expiration } \\
\text { Date }\end{array}$ & $\begin{array}{l}\text { Amount Approved } \\
\text { (SDR Million) }\end{array}$ & $\begin{array}{l}\text { Amount Drawn } \\
\text { (SDR Million) }\end{array}$ \\
\hline $\mathrm{ECF}$ & $\overline{\text { Nov } 14,2011}$ & $\overline{\text { Nov } 13,2014}$ & 85.00 & 12.00 \\
\hline $\mathrm{ECF}^{1}$ & Jun 26, 2006 & Sep 25,2010 & 81.00 & 75.35 \\
\hline
\end{tabular}

VI. Projected Payments to Fund ${ }^{2}$ SDR million

\begin{tabular}{|c|c|c|c|c|c|}
\hline & 2012 & 2013 & $\underline{2014}$ & $\underline{2015}$ & 2016 \\
\hline Principal & 2.45 & 8.29 & 12.81 & 14.51 & 15.07 \\
\hline Charges/Interest & 0.02 & 0.04 & 0.21 & 0.17 & 0.14 \\
\hline Total & 2.47 & 8.33 & 13.02 & 14.68 & 15.21 \\
\hline
\end{tabular}

VII. Implementation of HIPC Initiative:

Enhanced

I. Commitment of HIPC assistance

Framework

Decision point date

Jul 2007

Assistance committed by all creditors (US $\$$ million, NPV) ${ }^{3}$

582.40

Of which: IMF assistance (US\$ million)

Completion point date

Jan 2010

II. Disbursement of IMF assistance (SDR million)

Total disbursements

VIII. Implementation of MDRI Assistance: Not Applicable

\footnotetext{
${ }^{1}$ Formerly PRGF.

${ }^{2}$ Forthcoming; based on existing use of resources and present holdings of SDRs.

${ }^{3}$ Net Present Value (NPV) at the decision point under the enhanced framework.
} 


\section{Exchange Arrangement}

\section{Nonfinancial Relations}

Afghanistan is an Article XIV member country. The authorities are implementing a liberal exchange system. Based on information currently available to the staff, no exchange restrictions and multiple currency practices are in place. The authorities have provided documents to Fund staff related to laws and regulations on the exchange regime and have requested technical assistance from the Fund to formalize the current liberal regime. They have been implementing a managed float system with no predetermined path for the exchange rate. On May 31, 2012, the average of the buying and selling exchange rates in cash transactions on the Kabul money exchange market was 50.1950 Afghanis per U.S. dollar.

To conduct monetary policy, the authorities rely on foreign exchange auctions since May 2002, and on short-term capital note auctions since September 2004. The foreign exchange auctions were initially open only to licensed money changers, but since June 2005 they are also open to commercial banks. The capital note auctions are open to commercial banks. Auctions are linked to the overall monetary program and are held on the regular basis.

\section{Article IV Consultation}

The last Article IV consultation with Afghanistan was discussed by the Executive Board on November 14, 2011. Article IV consultation with Afghanistan are held in accordance with the decision on consultation cycles approved on July 15, 2002.

\section{Safeguards Assessment}

An update safeguards assessment of Da Afghanistan Bank (DAB) was finalized in December 2011. It found that while most of the previous safeguards recommendations had been implemented, an effective internal audit mechanism had still not been established and governance oversight was weak. The assessment also made recommendations to address new risks emerging as a result of the Kabul Bank crisis including with respect to central bank autonomy. Since the assessment, some recommendations have been implemented, albeit with delay. In particular, a Memorandum of Understanding on central bank capitalization has been signed and an external auditor has been appointed. The DAB is committed to implementing the remaining safeguards recommendations, with priority assigned to development of the internal audit function (with external support) and strengthening of Audit Committee oversight. 


\section{Technical Assistance, 2006-12}

\begin{tabular}{|c|c|c|}
\hline Department & Dates & Purpose \\
\hline \multirow[t]{20}{*}{ FAD } & February 2004-May 2006 & Treasury advisor \\
\hline & February $21-28,2006$ & Public financial management (budget and treasury procedures) \\
\hline & March $1-4,2006$ & Public financial management (budget and treasury procedures) \\
\hline & April 30-May 9, 2006 & Tax policy \\
\hline & November $14-23,2006$ & Tax and customs administration \\
\hline & October 23-November 27, 2008 & Resident public financial management advisor \\
\hline & July 4-16, 2009 & Organizational structure \\
\hline & December 2-12, 2009 & Revenue administration \\
\hline & December 6-16, 2009 & Program budgeting reform \\
\hline & April 3-17, 2010 & Revenue administration (customs) \\
\hline & April 6-19, 2010 & Program budgeting reform \\
\hline & June $6-19,2010$ & Revenue administration (customs) \\
\hline & September $14-27,2010$ & Tax policy reform \\
\hline & October $16-28,2010$ & Public financial management (with METAC) \\
\hline & January 22-February 4,2011 & Customs administration \\
\hline & April 1-13,2011 & Customs administration \\
\hline & September 15-22, 2011 & Program budgeting \\
\hline & January $14-26,2012$ & Revenue administration (value added tax) \\
\hline & January $14-28,2012$ & Customs reform \\
\hline & April 4-14, 2012 & Public financial management (with METAC) \\
\hline \multirow[t]{2}{*}{$\overline{L E G}$} & May $2-10,2006$ & Legal framework for monetary and payment systems \\
\hline & May $27-28,2010$ & Islamic banking law \\
\hline $\mathrm{MCD}$ & August 29, 2011-present & Resident monetary policy and banking advisor \\
\hline \multirow[t]{23}{*}{$\mathrm{MCM}$} & September 2004-April, 2006 & Training coordinator \\
\hline & January 22-February 5, 2006 & Monetary policy \\
\hline & April 2006-June 2007 & Training coordinator (peripatetic) \\
\hline & May $2-11,2006$ & Monetary policy, foreign reserve management, money markets \\
\hline & November 28-December 10, 2006 & Monetary policy formulation and implementation \\
\hline & March 19-April 16, 2007 & Central bank capacity building \\
\hline & March 24-29, 2007 & Banking supervision \\
\hline & April 17-30, 2007 & Monetary policy formulation and implementation \\
\hline & November 7-14, 2007 & Banking supervision \\
\hline & January $13-21,2008$ & Banking law (with LEG) \\
\hline & August 27-September 11, 2008 & Monetary policy formulation and implementation \\
\hline & August 2009 & Monetary operations \\
\hline & June $20-24,2010$ & Debt/money markets, sukuks (with METAC) \\
\hline & September 2010-present & Resident advisor on banking resolution \\
\hline & October 25-November 4, 2010 & Banking crisis management (with MCD) \\
\hline & February 7-14, 2011 & Banking crisis management (with MCD) \\
\hline & July $24-27,2011$ & Islamic finance (sukuk workshop) \\
\hline & October $1-12,2011$ & Banking supervision \\
\hline & February 11-14, 2012 & Islamic finance (sukuk) \\
\hline & April 7-12, 2012 & Banking supervision and regulations \\
\hline & April 15-17, 2012 & Islamic finance (sukuk) \\
\hline & May $27-30,2012$ & Islamic finance (sukuk) \\
\hline & June $23-28,2012$ & Banking supervision and regulations (PLANNED) \\
\hline \multirow[t]{2}{*}{ STA } & October 31, 2005-January 12, 2006 & Multisector statistics \\
\hline & March 6-15, 2006 & Balance of payments statistics \\
\hline
\end{tabular}




\begin{tabular}{|c|c|c|}
\hline & March 8-May 2, 2006 & Multisector statistics \\
\hline & March 13-22, 2006 & Monetary and financial statistics \\
\hline & May 24-July 18, 2006 & Multisector statistics \\
\hline & August 2-16, 2006 & Balance of payments statistics \\
\hline & August 8-October 3, 2006 & Multisector statistics \\
\hline & September 22-October 4, 2006 & Government finance statistics \\
\hline & November 1-December 26, 2006 & Multisector statistics \\
\hline & November 5-19, 2006 & Monetary and financial statistics \\
\hline & April 22-May 4, 2007 & Monetary and financial statistics \\
\hline & July 7-30, 2007 & National accounts \\
\hline & August 29-September 13, 2007 & Government finance statistics \\
\hline & September 09-October 05, 2007 & National accounts \\
\hline & December 02-13, 2007 & National accounts \\
\hline & April 20-30, 2008 & Monetary and financial statistics \\
\hline & August 6-27, 2008 & Balance of payments statistics \\
\hline & October 13-29, 2008 & National accounts \\
\hline & November 12-December 3, 2008 & Balance of payments statistics \\
\hline & November 23-December 5, 2008 & Monetary and financial statistics \\
\hline & August 30-September 9, 2009 & Monetary and financial statistics \\
\hline & September 27-October 07, 2009 & Balance of payments statistics \\
\hline & September $18-29,2010$ & Government finance statistics \\
\hline & September 20-October 1, 2010 & National accounts statistics \\
\hline & October 23-November 3, 2011 & Monetary statistics \\
\hline & April 29-May 10, 2012 & National accounts statistics \\
\hline \multirow{28}{*}{$\begin{array}{l}\text { (Afgha- } \\
\text { nistan is a } \\
\text { participant } \\
\text { in the } \\
\text { Middle } \\
\text { East } \\
\text { Technical } \\
\text { Assistance } \\
\text { Center) }\end{array}$} & May 2006 & Treasury modernization (resident treasury advisor) \\
\hline & June 8-13, 2006 & Budget integration \\
\hline & September 2006 & Banking supervision (training) \\
\hline & November, 2006 & Price statistics (training) \\
\hline & November $15-22,2006$ & Tax policy and administration \\
\hline & December 12-19, 2006 & Budget integration \\
\hline & December $12-22,2006$ & Budget development \\
\hline & March 10-21, 2007 & Tax policy and administration \\
\hline & March 11-22, 2007 & Cash management \\
\hline & March 24-29, 2007 & Banking supervision \\
\hline & May $19-25,2007$ & Balance of payments statistics \\
\hline & June 6-19, 2007 & Accounting \\
\hline & July 1-12, 2007 & Banking supervision \\
\hline & August 2007 & Cash management and program budgeting \\
\hline & September 1-12, 2007 & Banking supervision \\
\hline & November 4-8, 2007 & Banking supervision \\
\hline & January 5-26, 2008 & Central bank accounting \\
\hline & March 22-April 2, 2008 & Banking supervision \\
\hline & June 25-July 7, 2008 & Budget preparation \\
\hline & August $24-27,2008$ & Banking supervision \\
\hline & September 14-24, 2008 & Real sector statistics \\
\hline & December $14-17,2008$ & Banking supervision \\
\hline & January 25-February 2, 2009 & Central bank accounting \\
\hline & January 28-February 2, 2009 & Tax policy and administration \\
\hline & March 7-19, 2009 & Cash and budget management \\
\hline & February $13-24,2010$ & Public financial management \\
\hline & May $17-28,2010$ & Consumer price index \\
\hline & June $20-30,2010$ & Banking supervision (with MCM) \\
\hline
\end{tabular}




\begin{tabular}{|l|l|l|}
\hline & September 19-30, 2010 & Customs reform \\
\cline { 2 - 3 } & January 2-11,2011 & Financial planning, budget classification \\
\cline { 2 - 3 } & January 10-14, 2011 & Tax information systems (workshop) \\
\cline { 2 - 3 } & January 14-21,2012 & Revenue administration \\
\cline { 2 - 3 } & August 2-4, 2011 & Consumer price statistics \\
\cline { 2 - 3 } & December 10-19, 2011 & Consumer price statistics \\
\cline { 2 - 3 } & April 15-26, 2012 & Consumer price statistics \\
\cline { 2 - 3 } & June 25-28, 2012 & Balance of payments statistics (PLANNED) \\
\hline
\end{tabular}

\section{Resident Representatives}

Mr. de Schaetzen; August 2002-June 2005

Mr. Charap; June 2005-June 2008

Mr. Abdallah; June 2008-present 


\title{
ANNEX II. ISLAMIC REPUBLIC OF AFGHANISTAN-RELATIONS WITH THE WORLD BANK and Bank-Fund Collaboration Under the Joint Management ACtion Plan
}

\author{
(As of May 22, 2012)
}

1. IDA has committed a total of over US $\$ 2.3$ billion to Afghanistan since 2002. To date, 30 development and emergency reconstruction projects including 3 development policy grants have been implemented. IDA's active portfolio comprises 19 investment projects with combined net commitment of almost US $\$ 1$ billion, of which almost 50 percent (US\$478 million) has been disbursed.

2. The Bank's involvement in Afghanistan extends to its role as administrator of the Afghanistan Reconstruction Trust Fund (ARTF). The ARTF has developed into the primary multi-donor funding mechanism, financing the essential running costs of government as well as key investments, including national programs in health, education, rural access, irrigation rehabilitation, microfinance, and the National Solidarity Program. Since early 2002, 33 donors have contributed over US\$5.3 billion to this fund, making it the largest contributor to the Afghan budget for both operating costs and development programs.

3. In January 2010, the World Bank's International Development Association (IDA) and the International Monetary Fund (IMF) agreed to support debt relief for Afghanistan. The Boards of both institutions agreed that Afghanistan had taken the necessary steps to reach the completion point under the enhanced Heavily Indebted Poor Countries (HIPC) Initiative. This will generate total debt service savings of US $\$ 1.6$ billion, which include US $\$ 1.3$ billion from the HIPC Initiative, US\$260 million from Paris Club creditors beyond HIPC, and US\$38.4 million from the Multilateral Debt Relief Initiative (MDRI).

4. The International Finance Corporation (IFC), the World Bank Group's private sector development arm, continues to work with its investment partners in Afghanistan. IFC now has an investment portfolio totaling more than US $\$ 90$ million in six companies. This includes commitments in the financial, telecom, hospitality and healthcare sectors.

5. One of the primary focuses of the Bank's assistance has been to help the government with legal and regulatory reform, establishing fiscal authority, and developing systems and human resources for public financial management and the civil service as a whole. The performance of the Ministry of Finance regarding alignment of budgetary allocations with the country's priority needs, fiscal discipline, cash control, and aggregate transparency has contributed to macroeconomic stability and sustained external donor assistance.

6. In rural development, the National Solidarity Program (NSP) is active in 359 of Afghanistan's 364 districts and all of Afghanistan's 34 provinces. This is the Government of Afghanistan's flagship program aimed at strengthening Afghan communities' ability to identify, plan, manage and monitor their own development activities. As of May 2012 approximately 29,460 communities have been mobilized, of which 29,351 have elected 
Community Development Councils (CDCs). 29,294 CDCs have completed their Community Development Plans which have resulted in approximately 62,068 community subprojects being partially or fully financed, of which some 49,023 subprojects have been completed. About 80 percent of the community subprojects involve infrastructure such as irrigation, rural roads, electrification, and drinking water supply, all critical for the recovery of the rural economy, stability, and governance.

7. With support of the World Bank, the Ministry of Public Health nearly tripled the number of health facilities from 148 to 432 since 2003 in 11 provinces. About 85 percent of the population lives in districts, which now have service providers to deliver a basic package of health services. However, 57.4 percent of the population lives within one hour walking distance from a public health facility (AMS 2010). Health service utilization increased among project area populations from a rate of 0.3 consultations per capita annually at the outset to 1.21 per capita by mid 2011. Health care for expectant mothers expanded, with the number of deliveries assisted by trained health workers jumping from 14 percent to 34 percent (AMS2010). The number of pregnant women who received at least one prenatal care visit appears to have more than tripled from 16 percent to 60 between 2003 and 2010.

8. The demand for basic and higher education has continuously exceeded expectations and supply capacity. There are now 7.2 million children enrolled in schools, with nearly 40 percent being girls, compared to a little more than one million in 2001, with no girls. The Second Education Quality Improvement Project EQUIP, Strengthening Higher Education Project SHEP and the Afghanistan Skills Development Projects ASDP of the World Bank are geared towards providing equitable access to quality education and improving relevance of learning and skills of youth. Demand for education will continue to rise during the post transition decade.

9. Since the implementation of an Automated System for Customs Data, the collection of transit fees in major transit corridors in Afghanistan has improved and customs revenues have increased significantly over the past years. To date, customs processes have been automated at major Inland Customs Depots, including at the Kabul Airport which receives approximately 55 percent of all the country's customs declarations.

10. The Bank is helping to build the capacity of the government to exercise strong oversight of the minerals and hydrocarbons sectors. Private sector investment has been facilitated through the successful tender of the Aynak copper and Hajigak ore deposit. If these and other likely deposits are developed, foreign direct investment in the sector could amount to some US\$10-20 billion over the next decade.

11. Looking forward, the Bank's indicative lending envelope for 2012-13 is approximately US $\$ 260$ million. Resources will be primarily channeled to private and financial sector development as well as proven national programs in education and rural livelihood (including agriculture), where demands remain vast, to sustain and accelerate 
progress made so far. These areas constitute significant platforms for improving livelihoods and building government credibility.

12. Supporting sound national programs under the leadership of the government is the most effective use of international aid. The Bank will engage in areas where there is evidence: (i) that a strong, reform-minded, leadership is in place; (ii) where it can help leverage the support of other members of the international donor community; and (iii) where opportunities exist to develop operational frameworks for future programs. In this context, the Government has requested that the Bank play an enhanced role in working with them to determine the vision, design, and framework of their national programs.

13. Bank-Fund collaboration on macroeconomic issues proceeded according to the October 2011 memorandum under the Joint Management Action Plan. Both institutions worked closely on strengthening the banking sector and its supervision, as well as on issues related to Afghanistan's transition to more self-reliance, debt sustainability, fiscal sustainability and the macroeconomic framework in general. Inter alia, in support of Fund conditionality the Bank financed audits of ten weak banks and began implementation of the project to improvement the payments system. Table 1 below lists the Bank's and Fund's planned activities in the area of joint interest for June 2012-May 2013. 


\section{Table 1. Afghanistan: Bank's and Fund's Planned Activities in the Area of Joint Interest, June 2012-May 2013}

\begin{tabular}{|c|c|c|c|}
\hline & Products & Preparations/Mission Timing $^{1}$ & Delivery $^{1}$ \\
\hline \multirow[t]{4}{*}{ Fund } & First Review under the ECF & May 2012 & June/July 2012 \\
\hline & Second Review under the ECF & October 2012 & $\begin{array}{l}\text { December } \\
2012\end{array}$ \\
\hline & Third Review under the ECF & February 2013 & March 2013 \\
\hline & \multicolumn{3}{|c|}{$\begin{array}{l}\text { Areas of Technical Assistance: } \\
\text { ctor supervision (three long-term resident advisor), revenue } \\
\text { management, tax policy, public financial management, foreign } \\
\text { anking law, treasury securities, statistics (national accounts, prices, } \\
\text { ance of payments, GDDS) }\end{array}$} \\
\hline \multirow[t]{7}{*}{ Bank } & Development Policy Grant I & $\begin{array}{l}\text { November } 2011 \text { - September } \\
2012\end{array}$ & $\begin{array}{l}\text { September } \\
2012\end{array}$ \\
\hline & Financial Sector Strengthening Project, Supervision & November 2011 - August 2012 & October 2011 \\
\hline & ARTF IP Program (SY 1391), Preparation of MoU & November 2011 - June 2012 & June 2012 \\
\hline & Study: Long Term Sources Economic Growth & November 2011 - August 2012 & August 2012 \\
\hline & ESW: Sub-National Finance & August 2012 - September 2013 & $\begin{array}{l}\text { September } \\
2013\end{array}$ \\
\hline & DPG support to management of mineral revenues & January 2012 - September 2013 & February 2013 \\
\hline & Economic Monitoring & Continuous & $\begin{array}{l}\text { September } \\
2012 \text { and April } \\
2013\end{array}$ \\
\hline \multirow[t]{6}{*}{ Joint } & Financial Sector Assessment Program & TBD & TBD \\
\hline & $\begin{array}{l}\text { Dialogue on mineral revenue and expenditure } \\
\text { management }\end{array}$ & Continuous & \\
\hline & Dialogue on the transition process & Continuous & \\
\hline & Strengthening of the banking sector & Continuous & \\
\hline & Strengthening of customs & Continuous & \\
\hline & Improving payment systems & Continuous & \\
\hline
\end{tabular}

\footnotetext{
${ }^{1}$ Timing is tentative.
} 


\section{ANNEX III. ISLAMIC REPUbLiC OF AFGHANISTAN-RELATIONS WITH THE ASIAN DEVELOPMENT BANK}

(As of May 22, 2012)

1. A new Country Partnership Strategy (CPS) covering the 2009-2013 period was endorsed by ADB's Board of Directors on March 5, 2009. The new CPS is fully aligned with the Afghanistan National Development Strategy (ANDS), with ADB's investments contributing to Afghanistan's further economic development. The new CPS continues ADB's narrow focus on Afghanistan's energy, transport, and agriculture and irrigation sectors, including sector governance and further institutional and human capacity development.

2. As of end-December 2011 the Asian Development Bank had provided US $\$ 2.7$ billion to Afghanistan's reconstruction through approved Asian Development Fund (ADF) loan- and grant-financed projects, technical assistance, ADB administered Japan's Fund for Poverty Reduction (JFPR), and ADB private sector investments. Since 2007, all of ADB's public sector assistance has been provided on a 100 percent grant basis. At the June 2008 International Conference in Support of Afghanistan, ADB pledged an additional US $\$ 1.3$ billion over the 2008-2013 period, making ADB Afghanistan's fourth largest donor to Afghanistan (after the United States, Japan, and the European Union).

3. Since November 2008, ADB has approved four multi-tranche financing facility (MFF) projects as follows, a US\$570 million energy sector MFF and a US\$400 million transport sector MFF (November 2008); a US $\$ 805$ million transport sector MFF (October 2011), a US\$300 million water resources management sector MFF (October 2009); and a railway project of US $\$ 165$ million (October 2009). With the approval of these MFFs and the railway project, the bulk of ADB's anticipated assistance over the 2008-2013 period will be fully "earmarked," although with some flexibility depending on changes in the Government's priorities.

4. Since ADB operations in Afghanistan resumed in 2002, there have been 11 public sector loans in total US\$722.2 million and 19 grants in total US\$1580.1 million covering 23 investment projects. This involved ADF funding resources using loan or combined loan and grant funding, and since 2007 all projects have used only ADF grant-financing. In addition, there have been 10 Japan JFPR projects, 8 standalone projects, and 2 combined with loan projects totaling US\$110.0 million. Also, there have been 5 donor cofinancing grants from Japan's JFPR, Kuwait Fund, CIDA (2), and United Kingdom amounting to US\$76.7 million. ADB's private sector operations in Afghanistan began in 2004. By end of 2011, cumulative approvals for seven private sector projects have amounted to US\$206.1 million, including support for Afghanistan International Bank, Roshan Telecommunications, and the Afghanistan Investment Guarantee Facility.

5. ADB has been actively engaged in providing analytical and advisory technical assistance (TA) services to the Government, with total TA amounting to some 
US\$66.9 million as of the end 2011. Increasingly, TA is included as integral parts of ADB's grant-based investment projects, with focus on public financial management, procurement, and anti-corruption as well as institutional and human capacity development. ADB has also been providing ongoing TA support to the Ministry of Finance.

6. In November 2010, ADB approved the ADB-managed Afghanistan Infrastructure Trust Fund (AITF) to pool donor resources to cofinance and complement ADB-assisted infrastructure projects. AITF promotes infrastructure development by allowing donors to leverage ADB's technical expertise, fiduciary arrangements, and local capacity. To date, ADB has received cash flows amounting to US\$58 million from Japan and United Kingdom, with US\$33 million committed during 2011. Japan approved a further US\$50 million contribution to AITF in March 2012. ADB is also discussing the prospect of AITF contributions with the Australia, E.U., Germany, India, United States, and other interested partners. It is hoped that US\$1 billion could be raised for AITF to support the transition process.

7. $\mathrm{ADB}$ is a member of the Afghanistan Compact/ANDS Joint Coordination Management Board (JCMB) and also is a member of the Afghanistan Reconstruction Trust Fund Management Committee. ADB plays an active part in other donor coordination activities, including the JCMB Social and Economic Development Standing Committee, the Ministry of Finance's High Level Committee on Aid Effectiveness, and the Inter-Ministerial Committee on Energy. 
INTERNATIONAL MONETARY FUND

ISLAMIC REPUBLIC OF AFGHANISTAN

Joint World Bank/IMF Debt Sustainability Analysis Update

Prepared by the Staffs of the International Monetary Fund and the

International Development Association

June 19, 2012

Afghanistan continues to be at a high risk of debt distress. ${ }^{1},{ }^{2}$ Following debt relief under the enhanced HIPC initiative and MDRI, Afghanistan's external and public debt burden indicators have improved. However, and as noted in the HIPC Initiative Paper prepared in early $2010^{3}$ and the November 2011 debt sustainability analysis, ${ }^{4}$ debt burden indicators could deteriorate rapidly if Afghanistan's substantial financing needs were met with new loans, even concessional ones, instead of the hoped for grant financing, which would be discussed at the upcoming Tokyo Conference. Afghanistan's vulnerability is illustrated by the country-specific alternative scenarios.

\section{A. Macroeconomic Outlook}

1. The growth outlook underlying the DSA remains cautiously optimistic and is predicated on a stable security situation and grant-financed social and development spending. Real GDP growth rates are projected to reach an average of 5.5 percent during 2013-20 and to decline to about 4 percent thereafter (Box 1). ${ }^{5}$ The mining sector, as part of

\footnotetext{
${ }^{1}$ The results presented here are based on an update of the debt sustainability analysis based on the joint IMF/World Bank debt sustainability framework for low-income countries (see http://www.imf.org/external/pubs/ft/dsa/lic.htm and http://www.imf.org/External/np/pp/eng/2005/032805.htm).

${ }^{2}$ The LIC DSA compares the evolution over the projection period of debt-burden indicators against policydependent indicative thresholds, using the three-year average of the World Bank's Country Policy and Institutional Assessment (CPIA). With an average 2010 CPIA of 2.5, Afghanistan is classified as a "weak performer" according to the Debt Sustainability Framework (DSF).

${ }^{3}$ Afghanistan: HIPC Initiative Paper, IMF Country Report No. 10/40 (http://www.imf.org/external/pubs/ft/scr/2010/cr1040.pdf); and Memorandum and Recommendation of the President of the International Development Association to the Executive Directors on Assistance to the Islamic Republic of Afghanistan under the Enhanced Heavily Indebted Poor Countries Initiative and the Multilateral Debt Relief Initiative Report No.51184-AF.

${ }^{4}$ IMF Country Report No. 11/330 (http://www.imf.org/external/pubs/ft/scr/2011/cr11330.pdf).

${ }^{5}$ GDP estimates do not reflect opium production.
} 
industry, is expected to become an important contributor to growth, in addition to agriculture and services. To support sustainable inclusive growth, Afghanistan's business environment and economic governance will need to be strengthened significantly.

\section{Over the medium term, domestic revenue is expected to be boosted by tax} measures and mining revenues. The implementation of a VAT in 2014 could generate about 2 percent of GDP. In addition, the updated DSA incorporates the introduction of excises in 2018, yielding about 1 percent of GDP, to achieve the authorities' revenue target presented at the Bonn Conference in December 2011; alternative revenue measures could be considered. Fiscal revenue stemming from mining projects could reach 2 percent of GDP by 2020 , but there is significant uncertainty over the timing and level of these revenues. With these measures and developments in place, domestic revenue could reach 17 percent of GDP by 2025; a level that is in the upper range of what comparable countries achieve.

\begin{tabular}{|c|c|c|c|c|c|c|c|}
\hline \multicolumn{8}{|c|}{ Box 1. Macroeconomic Assumptions Comparison Table $1 /$} \\
\hline & \multicolumn{2}{|c|}{ DSA Nov. 2011} & \multicolumn{3}{|c|}{ DSA June 2012} & \multicolumn{2}{|c|}{$\begin{array}{c}\text { Differences } \\
\text { (current vs. previous) } \\
\end{array}$} \\
\hline & 2011-15 & 2016-30 & 2012 & $2012-16$ & $2017-32$ & Medium term & Long term \\
\hline Real growth (\%) & 6.1 & 4.6 & 6.4 & 5.9 & 4.4 & -0.2 & -0.2 \\
\hline Inflation (GDP deflator, \%) & 6.2 & 4.3 & 9.0 & 6.1 & 5.0 & -0.1 & 0.7 \\
\hline Nominal GDP (Bil. Afghani) & 1082 & 2947 & 1028 & 1298 & 3586 & 216 & 639 \\
\hline Revenue and grants (\% GDP) & 24.0 & 22.9 & 17.2 & 29.9 & 35.1 & 5.9 & 12.2 \\
\hline Grants (\% GDP) & 11.4 & 5.5 & 8.9 & 17.4 & 18.5 & 6.1 & 13.0 \\
\hline Primary expenditure (\% GDP) & 25.1 & 24.9 & 17.4 & 30.3 & 36.4 & 5.2 & 11.4 \\
\hline Primary deficit (\% GDP) & -1.2 & -2.1 & -0.3 & -0.4 & -1.2 & 0.8 & 0.8 \\
\hline Exports of G\&S (\% GDP) & 16.0 & 22.4 & 15.8 & 14.8 & 24.5 & -1.2 & 2.2 \\
\hline Imports of G\&S (\% GDP) & 48.4 & 32.4 & 60.9 & 54.8 & 47.4 & 6.5 & 15.0 \\
\hline Noninterest current account deficit (\% GDP) & -2.6 & -1.5 & 2.2 & -1.3 & -2.0 & 1.3 & -0.5 \\
\hline
\end{tabular}

\section{Expenditures will be driven by security, the take-over of the recurrent costs} associated with donor projects, and the size of the civil service, and development needs:

- $\quad$ Security-related outlays are estimated by the International Security Assistance Force (ISAF) to reach US\$4.1 billion in 2017 (or 14 percent of GDP). These projections assume that the size of the security forces is reduced by about one third in 2015-16about 120,000 troops. The authorities believe that such a sharp reduction may not be feasible over such a short period of time. If the troop size stayed at 350,000, security costs would be about US\$700 million higher in 2017 (2⿺辶2 percent of GDP). Beyond 2017 and with a constant troop size, security costs would decline only marginally as a share of GDP.

- $\quad$ Operating expenditures are expected to rise by a cumulative 4-5 percent of GDP by 2018, as the government assumes responsibility for previously donor-funded projects. 
In addition, operating expenditures will be pushed up by increases in the size of the civil service, mainly in the education and health sectors. At the same time, wage growth is projected quite conservatively at 1 percentage point over inflation, implying that the civil service would not benefit in full from the gains of real GDP growth. This may create wage pressures and could lead to even higher spending.

- Given Afghanistan's large development needs, the government is assumed to target development spending of 10 percent of GDP.

4. In view of these revenue and expenditure trends, Afghanistan's total financing needs are expected to remain higher than those of comparable low-income countries. Over the long term, the overall deficit (excluding grants) will remain at around 25 percent of GDP (Figures 1 and 2). A small share of this is likely to be financed from external concessional loans, and there is also some scope for domestic financing through the sukuk instrument that will be introduced in 2014 - mainly for market development and liquidity management purposes. The remaining financing gap is expected to stabilize at just over 20 percent of GDP in 2020 and beyond.

5. In this scenario, Afghanistan would continue to rely on donor support even for nonsecurity operating spending. Fiscal sustainability—defined as domestic revenues covering operating expenditures - is becoming a more distant goal, likely to be reached only after 2032. In the November 2011 DSA, fiscal sustainability was projected to be reached in 2025. The reason for this deterioration in outlook is that the current projections - based on better information - now incorporate the full operations and maintenance costs related to donor-funded projects in both security and the nonsecurity sectors. In the November 2011 DSA, this had been included as a risk since full information on the cost implications was not available.

\section{The scenario desribed above is only viable if donors meet the government's} financing needs of 20 percent of GDP with grants through 2025 and beyond. The May 2012 Chicago NATO summit fell short by US $\$ 300$ million of the US $\$ 4.1$ billion target considered necessary to cover Afghanistan's security spending needs through 2017, implying that security remains underfunded. The summit also asked that the government of Afghanistan should assume full responsibility for its financing needs by 2024 , hence eliminating its dependency on donor aid in this sector. While a progressive increase in Afghanistan's contribution to the financing of its security needs is incorporated in the scenario, full financing of security by domestic revenues could only be achieved by (i) cutting security spending, or (ii) reallocating substantial domestic resources toward security spending at the cost of development spending, leaving the latter significantly underfunded. This increases the pressure on development assistance, which will be discussed at the Tokyo Conference on July $8^{\text {th }}$. At the time of writing, the authorities were still finalizing their own projections and financing request for this conference. 
Figure 1. Afghanistan: Current Account Balance Excluding Grants (In percent of GDP)

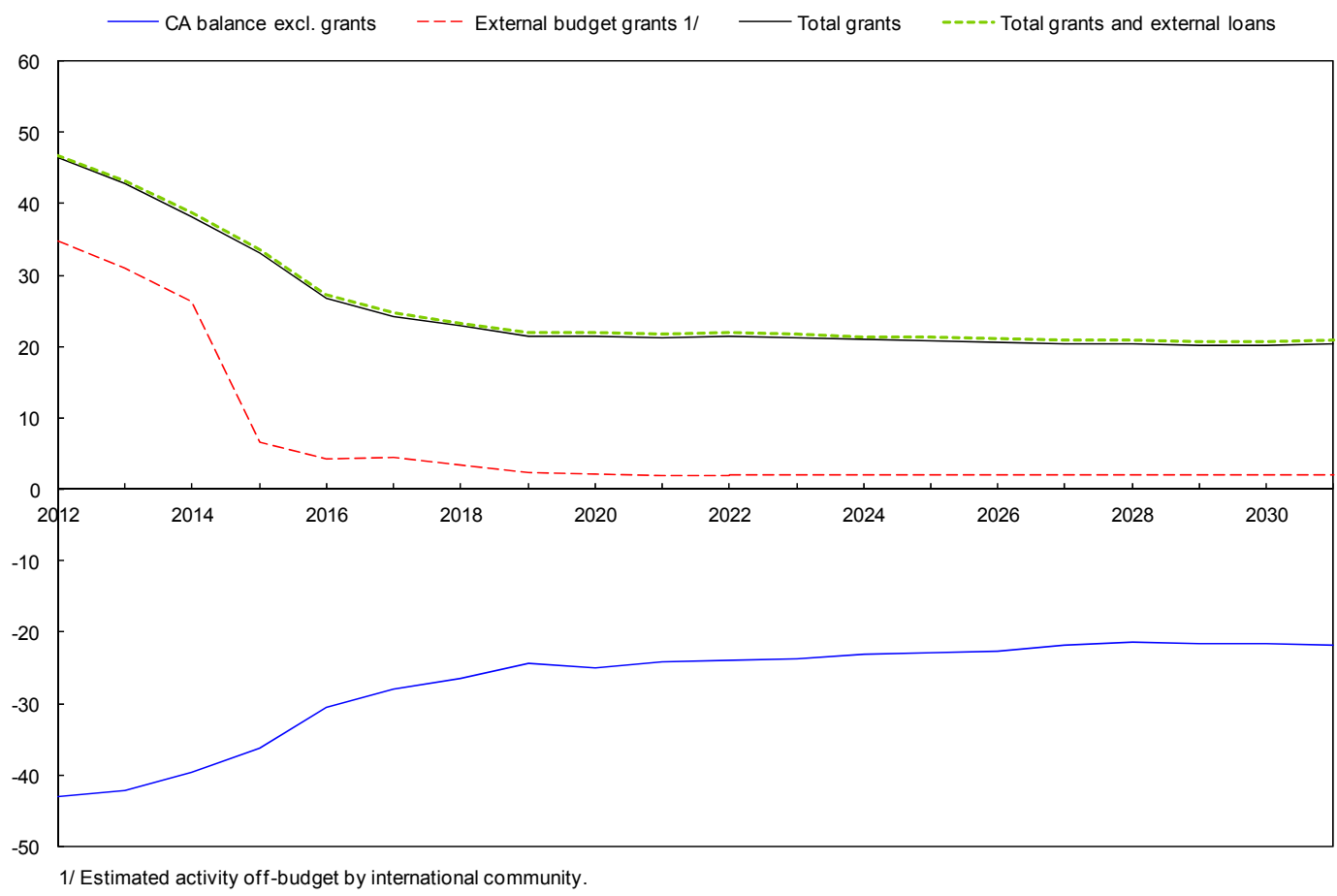

Figure 2. Afghanistan: Current Account Balance Including Grants (In percent of GDP)



CInternational Monetary Fund. Not for Redistribution 
7. In case donor funding should not be forthcoming over the longer term in the amounts needed to fill the financing gap, the authorities will face difficult decisions. Any grant shortfall will have to be met by additional revenue measures, which may be limited, or by spending cuts. Such fiscal consolidation is likely to weigh on short-term and long-term growth. There is only very limited scope to substitute debt financing for grants.

8. Given the large trade-off between civilian and development spending, the authorities should conduct regular public expenditure reviews to ensure that the expenditure mix remains appropriate. Under the baseline scenario, security spending remains high at 14.5 percent of GDP, compared to about 2-5 percent of GDP in other postconflict countries, though security situations are not easily comparable. The adequate provision of security services will remain a principal development objective in Afghanistan. However, over the years, the authorities should periodically review the country's security needs in line with developments. If the security situation were to be better than what underpins ISAF's projections, security spending might be lower than currently expected. In this context, the authorities can explore opportunities for more efficient, effective and transparent spending in all areas of government activity.

\section{B. Debt Sustainability Analysis}

9. Although Afghanistan's external and public debt burden indicators have improved significantly, following debt relief under the enhanced HIPC initiative and MDRI, the country remains at high risk of external debt distress. In 2011, Afghanistan's external public and publicly guaranteed debt amounted to US $\$ 1.2$ billion, or 7 percent of GDP, in 2011 (Tables 1 and 2). The bulk of this debt was owed to Paris Club and multilateral creditors. In present value terms, it reached about 4 percent of GDP at end-2011. Under the baseline scenario - in which Afghanistan's financing needs are fully met by grants - the present value of public external debt would reach about 5 percent of GDP by the end of the projection period, below the indicative debt-burden threshold applying to a country like Afghanistan. ${ }^{6}$ However, as set out in the alternative scenarios below, significant risks remain.

10. The first alternative scenario depicts a deterioration of the fiscal outlook with more limited access to grants. As a proxy for the above, Tables $3 a, 3 b$, and 4 and Figures 3 and 4 present the results of a customized alternative "lower grants" scenario in which grants are expected to fall short by 50 percent compared to the baseline. This implies that Afghanistan would still receive about 10 percent of GDP in grants, the upper range for comparable low-income countries. The government is assumed to have access to concessional loans, and hence the economy adjusts by borrowing both externally and domestically to offset the shortfall in grants. As a result, Afghanistan would face a rapid deterioration of debt burden indicators, with the PV of the debt-to-GDP ratio reaching 105 percent, the PV of the debt-to-exports ratio projected at 628 percent, and the PV of debt-

\footnotetext{
${ }^{6}$ Afghanistan is classified as a "weak performer" and its thresholds therefore: 30 percent of NPV for the debtto-GDP ratio; 100 percent of NPV for the debt-to-exports ratio; 200 percent for the debt-to-revenue ratio; 15 percent for the debt service-to-exports ratio; and 25 percent for the debt service-to-revenue ratio.
} 
to-revenue ratio reaching exceeding 600 percent by the end of the projection period. Other thresholds would also be breached. A shortfall in grants may also entail external adjustment needs, i.e. pressure on the exchange rate, which would add to the debt burden.

\section{Risks to the outlook are tilted to the downside, linked to security prospects and} the strength of future reforms. ${ }^{7}$ A fragile security situation, in combination with delays in key reforms, would potentially discourage investment and external support, and also result in lower exports, as well as a slowdown in revenue effort. Under the second alternative scenario, real GDP growth falls from 3 percent on average (instead of 5.5 percent in the baseline) in the medium-term (2013-20) to 1 percent (instead of 4 percent in the baseline) in the long-term (2025-30). Tables 3a, 3b, and 4 and Figures 3 and 4 present the results of a customized alternative "low-growth" scenario, depicting the absence of gains in security, governance, and public sector reforms, as well as a slower reform path with respect to financial sector reform and the business environment. Under this scenario, the PV of debt-toGPD ratio approaches 200 percent, while the PV of debt-to-revenue ratio is expected to reach 434 percent by the end of the forecast period, leaving very limited fiscal space for investments or social expenditure. Accordingly fiscal debt indicators set out on an explosive path, threatening the sustainability of government functions.

\section{Conclusions}

\section{Afghanistan remains at high risk of debt distress after the HIPC completion} point and delivery of debt relief under the MDRI. Despite the substantial amount of debt forgiven under the HIPC and MDRI, Afghanistan's very high security and development spending needs as well as risks to the macroeconomic outlook underscore the importance of substantial long-term grant financing, in combination with a strong reform agenda and progress in security and governance. Should donor support be insufficient to meet the country's financing needs, security fail to stabilize, or structural reforms and governance improvements fail to materialize, Afghanistan's debt burden would become unsustainable, and the government would be forced to undertake significant fiscal adjustment. As such, the government needs to prioritize very carefully its spending needs and avoid rapid expenditure increase until continuous financing has been clearly identified or domestic revenue mobilization picks up commensurately.

\footnotetext{
${ }^{7}$ This DSA does not include the standard stress tests, as these would not be characterized by additional vulnerabilities.
} 
Figure 3. Afghanistan: Indicators of Public and Publicly Guaranteed External Debt under Alternatives Scenarios, 2012-2032
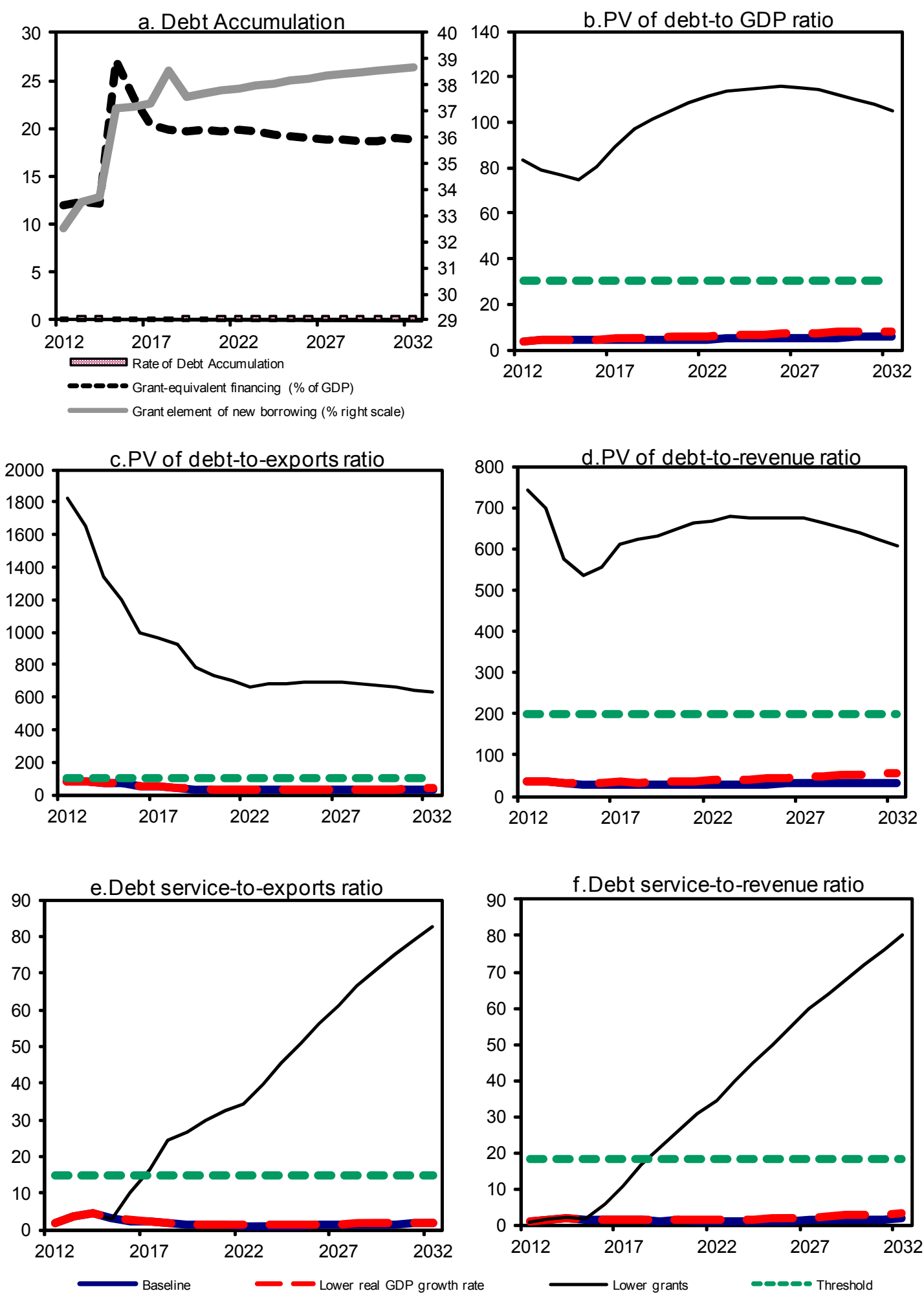

Sources: Country authorities; and staff estimates and projections. 
Figure 4. Afghanistan: Indicators of Public Debt Under Alternative Scenarios, 2012-2032


Sources: Country authorities; and staff estimates and projections.

1/ Revenues are defined inclusive of grants. 
Table 1. Afghanistan: External Debt Sustainability Framework, Baseline Scenario, 2009-2032 1

(In percent of GDP, unless otherwise indicated)

\begin{tabular}{|c|c|c|c|c|c|c|c|c|c|c|c|c|c|c|c|}
\hline & \multicolumn{3}{|c|}{ Actual } & \multirow{2}{*}{$\begin{array}{l}\text { Historical }{ }^{6 /} \\
\text { Average }\end{array}$} & \multirow{2}{*}{$\begin{array}{l}\text { i/ Standard }{ }^{6 /} \\
\text { Deviation }\end{array}$} & \multicolumn{6}{|c|}{ Projections } & & \multirow{2}{*}{\multicolumn{2}{|c|}{$\begin{array}{cc}2018-2032 \\
2032 & \text { Average } \\
\end{array}$}} \\
\hline & 2009 & 2010 & 2011 & & & 2012 & 2013 & 2014 & 2015 & 2016 & 2017 & $\begin{array}{c}\text { 2012-2017 } \\
\text { Average }\end{array}$ & 2022 & & \\
\hline External debt (nominal) $1 /$ & 9.3 & 8.2 & 7.0 & & & 6.6 & 6.7 & 6.8 & 6.9 & 7.0 & 7.2 & & 7.4 & 8.4 & \\
\hline $\mathrm{o} / \mathrm{w}$ public and publicly guaranteed (PPG) & 9.3 & 8.2 & 7.0 & & & 6.6 & 6.7 & 6.8 & 6.9 & 7.0 & 7.2 & & 7.4 & 8.4 & \\
\hline Change in external debt & -20.5 & -1.1 & -1.2 & & & -0.4 & 0.0 & 0.2 & 0.1 & 0.1 & 0.2 & & 0.1 & 0.1 & \\
\hline Identified net debt-creating flows & -8.8 & -7.9 & -6.1 & & & -3.7 & -3.6 & -2.9 & -0.9 & -0.3 & 0.1 & & -0.3 & -0.5 & \\
\hline Non-interest current account deficit & -1.7 & -3.9 & -3.4 & -2.3 & 2.5 & -1.8 & -0.6 & 0.7 & 3.0 & 3.7 & 3.6 & & 2.4 & 1.2 & 2.1 \\
\hline Deficit in balance of goods and services & 76.7 & 62.6 & 58.3 & & & 58.4 & 54.8 & 50.0 & 46.6 & 40.4 & 37.5 & & 32.5 & 31.9 & \\
\hline Exports & 4.9 & 4.5 & 4.4 & & & 4.6 & 4.8 & 5.7 & 6.3 & 8.1 & 9.3 & & 16.9 & 16.8 & \\
\hline Imports & 81.6 & 67.1 & 62.7 & & & 62.9 & 59.6 & 55.8 & 52.9 & 48.5 & 46.7 & & 49.4 & 48.6 & \\
\hline Net current transfers (negative $=$ inflow) & -60.2 & -50.8 & -47.8 & -51.6 & 28.7 & -48.3 & -45.5 & -40.6 & -35.4 & -28.8 & -26.3 & & -23.2 & -21.5 & -22.6 \\
\hline o/w official & -57.6 & -48.5 & -45.7 & & & -46.3 & -43.6 & -38.8 & -33.6 & -27.1 & -24.6 & & -21.7 & -20.3 & \\
\hline Other current account flows (negative $=$ net inflow) & -18.2 & -15.7 & -13.8 & & & -11.8 & -9.9 & -8.7 & -8.1 & -7.9 & -7.5 & & -6.8 & -9.2 & \\
\hline Net FDI (negative $=$ inflow) & -2.4 & -2.0 & -1.7 & -2.2 & 1.4 & -1.6 & -2.7 & -3.4 & -3.7 & -3.8 & -3.4 & & -2.5 & -1.5 & -2.3 \\
\hline Endogenous debt dynamics 2 & -4.7 & -2.0 & -1.0 & & & -0.3 & -0.3 & -0.2 & -0.2 & -0.2 & -0.1 & & -0.2 & -0.2 & \\
\hline Contribution from nominal interest rate & 0.1 & 0.0 & 0.0 & & & 0.1 & 0.1 & 0.1 & 0.2 & 0.2 & 0.2 & & 0.1 & 0.1 & \\
\hline Contribution from real GDP growth & -5.3 & -0.6 & -0.4 & & & -0.3 & -0.4 & -0.4 & -0.4 & -0.3 & -0.3 & & -0.3 & -0.3 & \\
\hline Contribution from price and exchange rate changes & 0.4 & -1.4 & -0.7 & & & $\ldots$ & & $\ldots$ & $\ldots$ & $\ldots$ & $\ldots$ & & $\ldots$ & $\ldots$ & \\
\hline Residual (3-4) 3/ & -11.7 & 6.7 & 4.9 & & & 3.3 & 3.6 & 3.1 & 1.0 & 0.4 & 0.1 & & 0.4 & 0.6 & \\
\hline o/w exceptional financing & -8.5 & 0.0 & 0.0 & & & 0.0 & 0.0 & 0.0 & 0.0 & 0.0 & 0.0 & & 0.0 & 0.0 & \\
\hline PV of external debt $4 /$ & $\ldots$ & $\ldots$ & 4.0 & & & 3.9 & 4.0 & 4.1 & 4.2 & 4.2 & 4.3 & & 4.6 & 5.6 & \\
\hline In percent of exports & $\ldots$ & $\ldots$ & 90.6 & & & 85.3 & 83.1 & 71.6 & 66.2 & 52.1 & 46.7 & & 27.3 & 33.6 & \\
\hline PV of PPG external debt & $\ldots$ & $\ldots$ & 4.0 & & & 3.9 & 4.0 & 4.1 & 4.2 & 4.2 & 4.3 & & 4.6 & 5.6 & \\
\hline In percent of exports & $\ldots$ & $\ldots$ & 90.6 & & & 85.3 & 83.1 & 71.6 & 66.2 & 52.1 & 46.7 & & 27.3 & 33.6 & \\
\hline In percent of government revenues & $\ldots$ & $\ldots$ & 38.3 & & & 34.7 & 35.3 & 30.7 & 29.7 & 29.1 & 29.7 & & 27.7 & 32.7 & \\
\hline Debt service-to-exports ratio (in percent) & 2.4 & 1.0 & 1.0 & & & 1.8 & 3.5 & 4.4 & 3.1 & 2.5 & 2.1 & & 1.1 & 1.6 & \\
\hline PPG debt service-to-exports ratio (in percent) & 2.4 & 1.0 & 1.0 & & & 1.8 & 3.5 & 4.4 & 3.0 & 2.5 & 2.1 & & 1.1 & 1.6 & \\
\hline PPG debt service-to-revenue ratio (in percent) & 1.1 & 0.4 & 0.4 & & & 0.7 & 1.5 & 1.9 & 1.4 & 1.4 & 1.3 & & 1.1 & 1.6 & \\
\hline Total gross financing need (millions of U.S. dollars) & -497.6 & -930.1 & -922.9 & & & -665.8 & -697.5 & -583.2 & -114.6 & 29.0 & 116.1 & & 31.6 & -27.9 & \\
\hline Non-interest current account deficit that stabilizes debt ratio & 18.8 & -2.8 & -2.2 & & & -1.4 & -0.7 & 0.5 & 2.9 & 3.6 & 3.5 & & 2.3 & 1.1 & \\
\hline \multicolumn{16}{|l|}{ Key macroeconomic assumptions } \\
\hline Real GDP growth (in percent) & 21.0 & 8.4 & 5.8 & 8.8 & 6.0 & 5.2 & 6.5 & 5.9 & 5.9 & 5.3 & 4.2 & 5.5 & 4.4 & 4.0 & 4.4 \\
\hline GDP deflator in US dollar terms (change in percent) & -1.5 & 17.7 & 8.6 & 8.8 & 6.2 & 3.8 & 3.4 & 1.8 & 1.0 & 1.0 & 1.0 & 2.0 & 1.2 & 1.3 & 1.1 \\
\hline Effective interest rate (percent) $5 /$ & 0.5 & 0.5 & 0.5 & 0.4 & 0.2 & 1.1 & 1.9 & 2.3 & 2.5 & 2.5 & 2.4 & 2.1 & 1.2 & 1.3 & 1.3 \\
\hline Growth of exports of G\&S (US dollar terms, in percent) & -15.1 & 17.3 & 12.5 & 9.2 & 12.0 & 12.7 & 15.3 & 28.6 & 17.1 & 37.1 & 20.5 & 21.9 & 14.0 & 5.4 & 10.1 \\
\hline Growth of imports of G\&S (US dollar terms, in percent) & 6.4 & 4.9 & 7.4 & 11.5 & 6.1 & 9.6 & 4.2 & 0.9 & 1.4 & -2.4 & 1.5 & 2.5 & 7.7 & 5.6 & 5.8 \\
\hline Grant element of new public sector borrowing (in percent) & & & & $\ldots$ & $\ldots$ & 32.5 & 33.6 & 33.7 & 37.1 & 37.2 & 37.3 & 35.2 & 37.9 & 38.7 & 38.2 \\
\hline Government revenues (excluding grants, in percent of GDP) & 10.3 & 11.0 & 10.5 & & & 11.3 & 11.3 & 13.4 & 14.0 & 14.5 & 14.5 & & 16.7 & 17.2 & 16.8 \\
\hline Aid flows (in millions of US dollars) 71 & 1,276 & 1,748 & 1,799 & & & 2,394 & 2,680 & 2,873 & 6,854 & 6,210 & 5,763 & & 7,583 & 12,000 & \\
\hline o/w Grants & 1,276 & 1,748 & 1,798 & & & 2,376 & 2,659 & 2,850 & 6,829 & 6,183 & 5,734 & & 7,536 & 11,887 & \\
\hline o/w Concessional loans & 0.3 & 0.2 & 0.2 & & & 18.5 & 20.7 & 22.8 & 24.8 & 26.9 & 28.9 & & 46.6 & 113.4 & \\
\hline Grant-equivalent financing (in percent of GDP) 8/ & $\ldots$ & $\ldots$ & $\ldots$ & & & 12.0 & 12.3 & 12.2 & 27.1 & 23.1 & 20.4 & & 20.0 & 18.9 & 19.3 \\
\hline Grant-equivalent financing (in percent of external financing) 8/ & $\ldots$ & $\ldots$ & $\ldots$ & & & 98.2 & 96.3 & 96.2 & 98.7 & 98.5 & 98.3 & & 98.3 & 97.8 & 98.1 \\
\hline \multicolumn{16}{|l|}{ Memorandum items: } \\
\hline Nominal GDP (millions of US dollars) & 12,487 & 15,940 & 18,315 & & & 19,997 & 22,030 & 23,747 & 25,407 & 27,043 & 28,447 & & 38,171 & 63,883 & \\
\hline Nominal dollar GDP growth & 19.3 & 27.7 & 14.9 & & & 9.2 & 10.2 & 7.8 & 7.0 & 6.4 & 5.2 & 7.6 & 5.7 & 5.3 & 5.5 \\
\hline PV of PPG external debt (in millions of US dollars) & & & 713 & & & 770 & 867 & 958 & 1,037 & 1,119 & 1,206 & & 1,733 & 3,536 & \\
\hline (PVt-PVt-1)/GDPt-1 (in percent) & & & & & & 0.3 & 0.5 & 0.4 & 0.3 & 0.3 & 0.3 & 0.4 & 0.4 & 0.4 & 0.4 \\
\hline Gross workers' remittances (millions of US dollars) & 0.0 & 0.0 & 0.0 & & & 0.0 & 0.0 & 0.0 & 0.0 & 0.0 & 0.0 & & 0.0 & 0.0 & \\
\hline PV of PPG external debt (in percent of GDP + remittances) & & $\ldots$ & 4.0 & & & 3.9 & 4.0 & 4.1 & 4.2 & 4.2 & 4.3 & & 4.6 & 5.6 & \\
\hline PV of PPG external debt (in percent of exports + remittances) & $\ldots$ & $\ldots$ & 90.6 & & & 85.3 & 83.1 & 71.6 & 66.2 & 52.1 & 46.7 & & 27.3 & 33.6 & \\
\hline Debt service of PPG external debt (in percent of exports + remittance & $\ldots$ & $\ldots$ & 1.0 & & & 1.8 & 3.5 & 4.4 & 3.0 & 2.5 & 2.1 & & 1.1 & 1.6 & \\
\hline
\end{tabular}

Sources: Country authorities; and staff estimates and projections.

1/ Includes both public and private sector external debt.

2/ Derived as $[r-g-\rho(1+g)] /(1+g+\rho+g \rho)$ times previous period debt ratio, with $r=$ nominal interest rate; $g=$ real GDP growth rate, and $\rho=$ growth rate of GDP deflator in U.S. dollar terms.

$3 /$ Includes exceptional financing (i.e., changes in arrears and debt relief); changes in gross foreign assets; and valuation adjustments. For projections also includes contribution from price and exchange rate changes.

4/ Assumes that PV of private sector debt is equivalent to its face value.

6/ Historical averages and standard deviations are generally derived over the past 10 years, subject to data availability.

7/ Defined as grants, concessional loans, and debt relief.

8/ Grant-equivalent financing includes grants provided directly to the government and through new borrowing (difference between the face value and the PV of new debt).

CInternational Monetary Fund. Not for Redistribution 
Table 2. Afghanistan: Public Sector Debt Sustainability Framework, Baseline Scenario, 2009-2032

(In percent of GDP, unless otherwise indicated)

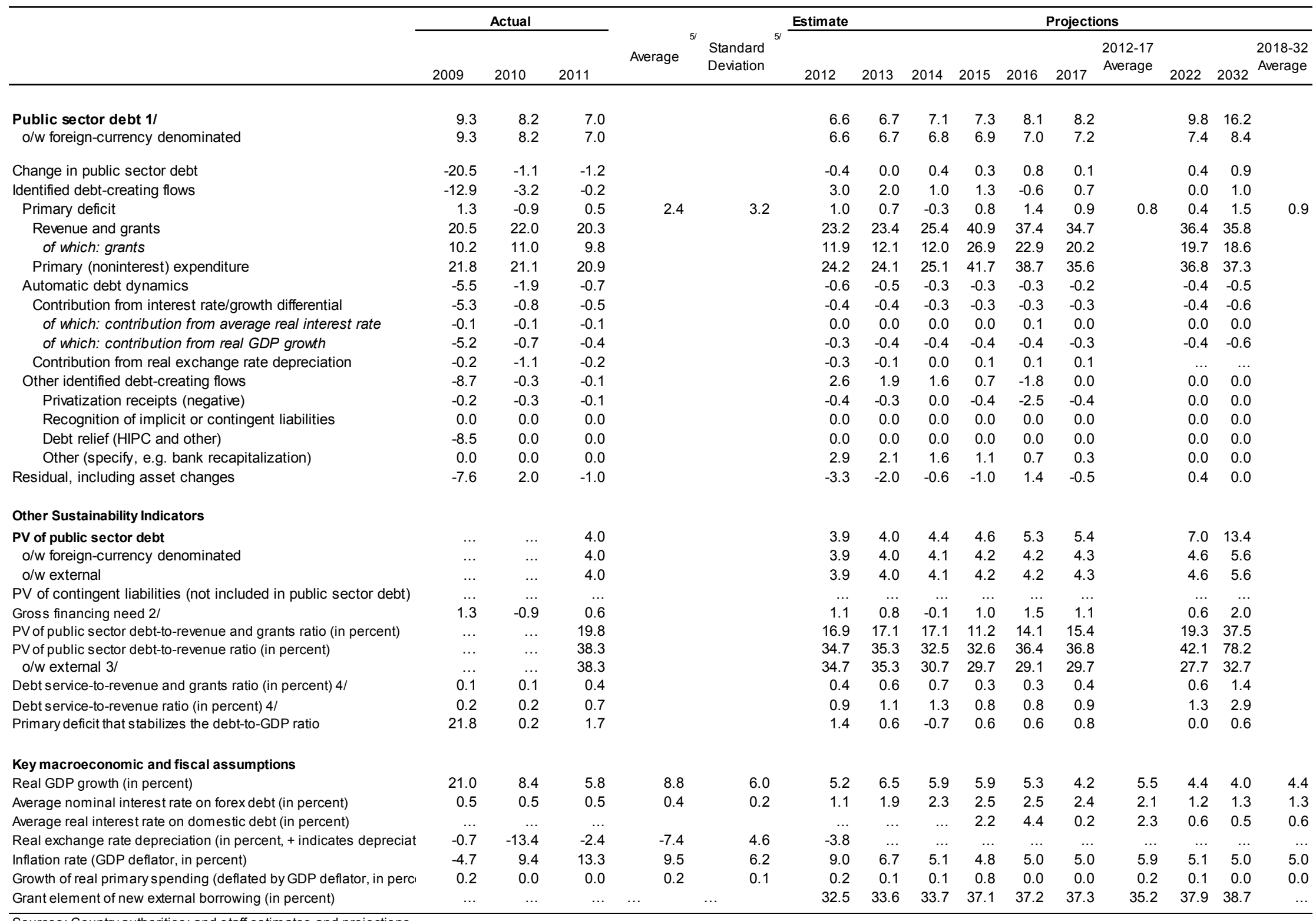

Sources: Country authorities; and staff es timates and projections.

$1 /$ Refers to net public debt of the central government.

2/ Gross financing need is defined as the primary deficit plus debt service plus the stock of short-term debt at the end of the last period.

$3 /$ Revenues excluding grants.

4/ Debt service is defined as the sum of interest and amortization of medium and long-term debt

5/ Historical averages and standard deviations are generally derived over the past 10 years, subject to data availability.

CInternational Monetary Fund. Not for Redistribution 
Projections

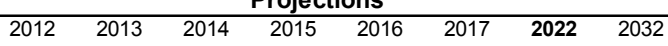

\section{PV of debt-to GDP ratio}

Baseline

A. Alternative Scenarios

A1. Lower real GDP growth rate

A2. Lower grants

B. Bound Tests

B1. Real GDP growth at historical average minus one standard deviation in 2013-2014

B2. Export value growth at historical average minus one standard deviation in 2013-2014 3/

B3. US dollar GDP deflator at historical average minus one standard deviation in 2013-2014

B4. Net non-debt creating flow $S$ at historical average minus one standard deviation in 2013-2014 4/

B5. Combination of B1-B4 using one-half standard deviation shocks

B6. One-time 30 percent nominal depreciation relative to the baseline in 20135 /
4

4

77

4

4

$4 \quad 20$

4

\section{PV of debt-to-exports ratio}

Baseline

85

A. Alternative Scenarios

A1. Lower real GDP growth rate

A2. Lower grants

B. Bound Tests

B1. Real GDP growth at historical average minus one standard deviation in 2013-2014

B2. Export value growth at historical average minus one standard deviation in 2013-2014 3/

B3. US dollar GDP deflator at historical average minus one standard deviation in 2013-2014

B4. Net non-debt creating flows at historical average minus one standard deviation in 2013-2014 4/

B5. Combination of B1-B4 using one-half standard deviation shocks

B6. One-time 30 percent nominal depreciation relative to the baseline in 2013 5/
1823
83

83

1647
73

1341

1192

999

967

$29 \quad 36$

$663 \quad 628$

PV of debt-to-revenue ratio

Baseline

A. Alternative Scenarios

A1. Lower real GDP growth rate

A2. Lower grants

\section{B. Bound Tests}

B1. Real GDP growth at historical average minus one standard deviation in 2013-2014 B2. Export value growth at historical average minus one standard deviation in 2013-2014 3/ B3. US dollar GDP deflator at historical average minus one standard deviation in 2013-2014

B4. Net non-debt creating flow s at historical average minus one standard deviation in 2013-2014 4/

B5. Combination of B1-B4 using one-half standard deviation shocks

B6. One-time 30 percent nominal depreciation relative to the baseline in 2013 /

$\begin{array}{rrrrrrrr}35 & 36 & 32 & 31 & 30 & 31 & \mathbf{2 9} & 34 \\ 35 & 39 & 44 & 42 & 40 & 40 & \mathbf{3 6} & 36 \\ 35 & 35 & 30 & 29 & 29 & 29 & \mathbf{2 7} & 32 \\ 35 & 173 & 238 & 221 & 209 & 205 & \mathbf{1 5 9} & 89 \\ 35 & 86 & 91 & 85 & 81 & 80 & \mathbf{6 5} & 47 \\ 35 & 49 & 42 & 41 & 40 & 41 & \mathbf{3 8} & 45\end{array}$


Table 3b. Afghanistan: Sensitivity Analysis for Key Indicators of Public and Publicly Guaranteed External Debt, 2012-2032 (continued) (In percent)

Debt service-to-exports ratio

\section{Baseline}

A. Alternative Scenarios

A1. Lower real GDP growth rate

A2. Lower grants

\section{B. Bound Tests}

B1. Real GDP growth at historical average minus one standard deviation in 2013-2014 B2. Export value growth at historical average minus one standard deviation in 2013-2014 3/ B3. US dollar GDP deflator at historical average minus one standard deviation in 2013-2014

B4. Net non-debt creating flow s at historical average minus one standard deviation in 2013-2014 4/

B5. Combination of B1-B4 using one-half standard deviation shocks

B6. One-time 30 percent nominal depreciation relative to the baseline in 20135 /

$\begin{array}{rrrrrrrr}2 & 3 & 4 & 3 & 3 & 2 & 1 & 2 \\ 2 & 3 & 4 & 3 & 10 & 17 & 34 & 83\end{array}$

\section{Debt service-to-revenue ratio}

Baseline

\section{A. Alternative Scenarios}

A1. Lower real GDP growth rate

A2. Lower grants

B. Bound Tests

B1. Real GDP growth at historical average minus one standard deviation in 2013-2014

B2. Export value growth at historical average minus one standard deviation in 2013-2014 3/

B3. US dollar GDP deflator at historical average minus one standard deviation in 2013-2014

B4. Net non-debt creating flow s at historical average minus one standard deviation in 2013-2014 4/

B5. Combination of B1-B4 using one-half standard deviation shocks

B6. One-time 30 percent nominal depreciation relative to the baseline in $20135 /$

Memorandum item:

Grant element assumed on residual financing (i.e. financing required above baseline) $6 /$

$\begin{array}{llllllll}1 & 2 & 2 & 1 & 1 & 1 & \mathbf{1} & 2 \\ 1 & 1 & 2 & 2 & 2 & 2 & \mathbf{1} & 2 \\ 1 & 1 & 2 & 1 & 1 & 1 & \mathbf{1} & 2 \\ 1 & 1 & 4 & 5 & 5 & 5 & \mathbf{7} & 7 \\ 1 & 1 & 3 & 2 & 2 & 2 & \mathbf{3} & 3 \\ 1 & 2 & 3 & 2 & 2 & 2 & \mathbf{2} & 2 \\ 36 & 36 & 36 & 36 & 36 & 36 & 36 & 36\end{array}$

Sources: Country authorities; and staff estimates and projections.

1/ Variables include real GDP growth, growth of GDP deflator (in U.S. dollar terms), non-interest current account in percent of GDP, and non-debt creating flows

2/ Assumes that the interest rate on new borrowing is by 2 percentage points higher than in the baseline., while grace and maturity periods are the same as in the baseline.


an offsetting adjustment in import levels).

4/ Includes official and private transfers and FDI.

5/ Depreciation is defined as percentage decline in dollar/local currency rate, such that it never exceeds 100 percent.

6/ Applies to all stress scenarios except for A2 (less favorable financing) in which the terms on all new financing are as specified in footnote 2. 
Table 4. Afghanistan: Sensitivity Analysis for Key Indicators of Public Debt 2012-2032

Baseline
A. Alternative scenarios
A1. Lower real GDP growth rate
A2. Lower grants
B. Bound tests
B1. Real GDP growth is at historical average minus one standard deviations in 2013-20.
B2. Primary balance is at historical average minus one standard deviations in 2013-201،
B3. Combination of B1-B2 using one half standard deviation shocks
B4. One-time 30 percent real depreciation in 2013
B5. 10 percent of GDP increase in other debt-creating flows in 2013

PV of Debt-to-Revenue Ratio 2/

\section{Baseline}

\section{A. Alternative scenarios}

A1. Lower real GDP growth rate

A2. Lower grants

$\begin{array}{rrrrrrrr}4 & 4 & 5 & 6 & 7 & 8 & 13 & 25 \\ 4 & 7 & 11 & 11 & 11 & 11 & 12 & 17 \\ 4 & 6 & 9 & 9 & 10 & 10 & 11 & 17 \\ 4 & 6 & 6 & 6 & 6 & 6 & 8 & 14 \\ 4 & 9 & 9 & 9 & 9 & 10 & 12 & 18\end{array}$

\section{B. Bound tests}

B1. Real GDP growth is at historical average minus one standard deviations in 2013-20.

B2. Primary balance is at historical average minus one standard deviations in 2013-201.

B3. Combination of B1-B2 using one half standard deviation shocks

B4. One-time 30 percent real depreciation in 2013

B5. 10 percent of GDP increase in other debt-creating flows in 2013

$\begin{array}{rrrrrrrr}4 & 4 & 5 & 7 & 10 & 12 & 44 & 191 \\ 84 & 79 & 81 & 91 & 100 & 109 & 131 & 124\end{array}$

Debt Service-to-Revenue Ratio 2/

\section{Baseline}

\section{A. Alternative scenarios}

A1. Lower real GDP growth rate

A2. Lower grants

$\begin{array}{llllllll}17 & 17 & 17 & 11 & 14 & 15 & 19 & 38\end{array}$

\section{B. Bound tests}

B1. Real GDP growth is at historical average minus one standard deviations in 2013-20.

B2. Primary balance is at historical average minus one standard deviations in 2013-201،

B3. Combination of B1-B2 using one half standard deviation shocks

B4. One-time 30 percent real depreciation in 2013

B5. 10 percent of GDP increase in other debt-creating flows in 2013

$\begin{array}{rrrrrrrr}17 & 17 & 20 & 18 & 24 & 34 & 107 & 434 \\ 361 & 338 & 379 & 312 & 364 & 407 & 445 & 423\end{array}$

$\begin{array}{llllllll}17 & 18 & 21 & 15 & 19 & 23 & 34 & 68 \\ 17 & 30 & 43 & 27 & 30 & 33 & 34 & 48 \\ 17 & 26 & 35 & 22 & 26 & 28 & 31 & 49 \\ 17 & 24 & 22 & 14 & 17 & 18 & 21 & 40 \\ 17 & 40 & 36 & 22 & 25 & 28 & 33 & 50\end{array}$

Sources: Country authorities; and staff estimates and projections.

$1 /$ Assumes that real GDP growth is at baseline minus one standard deviation divided by the square root of the length of the projection period.

2/ Revenues are defined inclusive of grants. 


\section{Statement by the IMF Staff Representative on the Islamic Republic of Afghanistan}

\section{June 29, 2012}

Since the staff report was finalized, the authorities provided staff with revised outcome data for domestic revenue collection, donor assistance grants, and foreign loans at end-March 2012 (Table 1). The revisions do not change the staff appraisal.

- The revised revenue collection is Afs 97.5 billion, which is Afs 0.2 billion ( 0.02 percent of GDP) higher than shown in the staff report and the authorities' letter of intent. Even with this revision, the end-March revenue target of Afs 98.8 billion was missed, and the quantitative performance criterion is not observed.

- $\quad$ The revised grants stand at Afs 93.5 billion, which is Afs 7.6 billion (1 percent of GDP) lower than shown in the staff report. The revised foreign loans stand at Afs 3.1 billion, which is Afs 1.5 billion ( 0.2 percent of GDP) higher than shown in the staff report and the authorities' letter of intent. These revisions lead to minor changes in the calculations of the adjustors to two quantitative program targets - net international reserves and net central bank credit to the government — but do not materially affect performance under the program. 
Press Release No. 12/245

FOR IMMEDIATE RELEASE

June 29, 2012
International Monetary Fund

Washington, D.C. 20431 USA

\section{IMF Executive Board Completes First Review Under the Extended Credit Facility Arrangement for the Islamic Republic of Afghanistan, Approves US\$18.2 Million Disbursement}

The Executive Board of the International Monetary Fund (IMF) today completed the first review of the Islamic Republic of Afghanistan's economic performance under the program supported by a three-year, SDR 85 million (about US\$129 million) Extended Credit Facility (ECF) arrangement (see Press Release No. 11/412).

Completion of the review makes SDR 12 million (about US\$18.2 million) available to the Islamic Republic of Afghanistan. This would bring total disbursements under the arrangement to SDR 24 million (about US\$36.4 million).

Executive Directors noted the progress achieved by the authorities on their reform objectives under the program during difficult times. In completing the review, the IMF's Board also approved the authorities' request for a waiver of nonobservance of a performance criterion on fiscal revenues of the central government, and the authorities' request for modification of performance criteria, as well as rephasing of disbursements.

Following the Executive Board's discussion, Ms. Nemat Shafik, Deputy Managing Director and Acting Chair, stated:

"In a very difficult environment, Afghanistan has begun a transition toward greater macroeconomic stability and economic self-reliance. The IMF-supported economic program has been designed to provide the policy frameworks for managing this transition and catalyzing support from the international donor community.

"Under their program, the Afghan authorities are pursuing important structural reforms. In particular, they have drafted a law for the introduction of a value-added tax in 2014, prepared a strategy to fight economic crimes, and strengthened the central bank's capitalization framework. The authorities have also reported on asset recovery from Kabul Bank where cash recoveries have increased following a presidential decree. However, no shareholder has paid in full, and their cases have been referred to a special tribunal and the Financial Dispute Resolution Committee. 
"Going forward, the program will continue to focus on safeguarding the financial sector, strengthening economic governance, and moving toward fiscal sustainability. While progress has been made on all these fronts, strong government ownership of the program remains crucial to resist opposition from vested interests.

"The authorities' development strategy rests on greater revenue mobilization and sustained strong donor support to meet large spending needs for security and development in the years ahead. They look to donors to firm up their aid pledges at the forthcoming Tokyo

Conference. The authorities will need to strengthen budget implementation capacity, address critical governance issues, and improve accountability within various government agencies." 


\section{Statement by Mr. Mojarrad, Executive Director for the Islamic Republic of Afghanistan and Mr. Jbili, Alternate Executive Director June 29, 2012}

On behalf of our Afghan authorities, we would like to thank management and staff for the constructive discussion for this first review of the ECF-supported program. All quantitative targets were met, with the exception of the one on revenue collection, which was missed by a small margin. Advances in structural reforms were achieved with five out of six structural benchmarks being completed. The authorities have requested completion of the first review and a waiver for the minor deviation from the quantitative target on revenue collection by the central government, for which the authorities are taking corrective action.

\section{Recent developments and prospects for 2012}

The economy grew by 6 percent in real terms in 2011/12, or slightly above program projections, which is significant given the poor harvest compared to the previous year. With a rebound in agricultural production in 2012, real GDP is expected to grow by 7 percent. Inflation decelerated further in 2011 to single digits, and is expected to be around $71 / 2$ percent in 2012, reflecting some easing of international commodity prices and the planned tightening of monetary policy. The operating fiscal deficit was contained at 6.1 percent of GDP in 2011/12, as operating expenditures were reduced in the face of a small revenue shortfall.

The current account deficit, excluding official transfers, increased due to trade disruptions with neighboring countries and higher fuel imports. The deterioration, however, was more than offset by donor support, leading to a larger-than-programmed current account surplus, including official transfers, and higher-than projected accumulation of international reserves.

\section{Program implementation}

When considering Afghanistan's request of a new ECF in support of its program in November 2011, the Executive Board recognized the considerable challenges lying ahead and stressed the importance of strong implementation. Indeed, Afghanistan continues to face severe adverse conditions, including among others, the dire security situation, the disruption of trade with neighboring countries, the economic fallout of the Kabul Bank problem, and the impact of the expected reduction in international presence in the country. Notwithstanding these difficulties, advances have been made in several areas.

In line with the program objectives, the authorities' efforts have focused on moving forward with the agreed strategy to resolve the Kabul Bank problem, while seizing this opportunity to address broader issues of financial sector reform and economic governance. They also stepped up efforts to achieve other objectives under the ANDS, including revenue mobilization, efficiency in public spending, fiscal sustainability, and poverty reduction.

The Afghan authorities have difficulties with the staff report's mixed messages. While the report indicates that "the authorities have made substantial progress on their reform objectives under the program," it also points out that "program implementation has been 
weak." The authorities disagree with the latter statement, which is not borne out by actual accomplishments since the program was approved, as detailed in the MEFP and the accompanying Table 1. While delays in tackling structural reforms and complex legal and financial problems are to be expected in a war-torn country with limited capacity, the authorities have taken decisive steps to achieve agreed objectives. Accordingly, they believe that delays in some instances should not be seen as reflecting lack of program ownership. More generally, the authorities are concerned that the unduly negative tone of the report could send the wrong signal to the Tokyo conference and risk undermining donor support to Afghanistan.

\section{Financial sector reform}

Resolving the Kabul Bank problem remains a top priority. New Kabul Bank is being readied for privatization, following approval by the cabinet, and an advisor has been appointed to this effect. The bank will be put up for sale in a transparent way to qualified bidders as specified in the structural benchmark for end-September 2012, or wound down by December 2013 if the sale does not materialize.

Following a difficult start, asset recovery from Kabul Bank beneficiaries has accelerated since April 2012 through cash payments and takeover of property. As detailed in the MEFP, further steps to strengthen asset recovery have been taken, including the issuance of a presidential decree on April 4, 2012, mandating that all Kabul Bank beneficiaries shall promptly repay amounts owed in full or face legal action, and establishing a special tribunal in coordination with the Supreme Court to hear Kabul Bank related cases. The authorities have initiated legal proceedings against each beneficiary for the amount indicated in the forensic report, and have brought forward criminal charges against the main architects of the fraud. The authorities have also accelerated civil collections against other beneficiaries, referring cases to the Financial Dispute Resolution Commission for action. Finally, the authorities have made a formal request for mutual legal assistance to the United Arab Emirates in connection with properties located in that country and to trace funds that may have passed through that jurisdiction. To foster transparency of the asset recovery process, progress will be posted on the Central Bank's website starting in September 2012. While the amount of recovered assets so far, including the book value of seized properties, is not insignificant, the process that has been put in place could yield more tangible results going forward. The authorities are committed to ensuring the success of the asset recovery program, which together with accompanying reforms, will send the right signal about the rule of law and the protection of the financial system.

Aside from Kabul Bank, efforts have focused on addressing other issues of safety, governance, and development of the financial sector (MEFP q21-24). On the basis of audits of 10 small banks, which revealed inadequate capitalization, deficiencies in governance, and excessive exposure, the authorities are developing strategies to address these issues, with implementation expected to start in December 2012. Recapitalization of the central bank is expected to be completed before 2014, and the Ministry of Finance and DAB have signed a 
memorandum of understanding to this effect. Efforts are also underway to upgrade the regulatory framework and strengthen DAB's supervision capacity.

Finally, the authorities have adopted a broad strategy aimed at combating economic crimes and strengthening cooperation among relevant government entities, and integrated that strategy into the National Priority Program "Justice for All." An Economic Crime Task Force is being established to coordinate the activities of various government entities related to economic crimes, including among others, enhancing the capacity of the justice system to deal with money laundering, terrorist finance, and asset recovery (MEFP q29). In addition, the authorities are taking steps to strengthen the AML/CFT framework in collaboration with the Financial Action Task Force (FATF). New AML/CFT laws will be submitted to parliament in September 2012, and implementation of an action plan agreed with FATF will start at that time (MEFP $\mid 30$ ).

\section{Structural reforms}

Progress in structural reforms has been made in several important areas as indicated by staff. In line with the authorities' strategy to broaden the tax revenue base and raise its share to GDP, a draft VAT law, prepared in consultation with Fund staff, has been submitted to the Ministry of Justice for review and will be sent to parliament for approval in December 2012. The VAT will apply to large taxpayers, who will be identified in the context of the preparatory work underway. To prevent the erosion of the tax base, all "concessions" for importers will be eliminated.

Development of the mining sector will generate significant revenue, which the authorities intend to channel to the budget in a transparent manner under a sound fiscal regime for mineral resources. Preparatory work for this regime is underway in collaboration with the Fund and the World Bank, leading to the submission of draft amendments to the appropriate laws to the ministry of justice by end-June 2013. The fiscal regime for mineral resources will be in full compliance with the Extractive Industries Transparency Initiative.

Efforts have also focused on expenditure, as detailed in the MEFP, including improvements in budget planning and implementation through introduction of budget planning, identification and reduction of fiscal risks stemming from state-owned enterprises, and improvement in cash management, through the planned issuance of Sukuks (Sharia compliant instruments).

\section{Macroeconomic policies}

Macroeconomic policies will continue to be guided by the need to achieve the ANDS objectives within the ECF-supported program framework, with a particular focus on revenue mobilization to cover an increasing share of operating spending, improved public financial management, lowering inflation, and achieving financial stability. The 2012 budget is in line with the program objectives, monetary policy is appropriately geared toward reducing inflation, and the floating exchange rate will continue to reflect market fundamentals, with 
DAB intervention limited to smoothing out volatility and achieving its reserve money and international reserves targets.

\section{Debt sustainability}

Afghanistan's financing needs in connection with the high cost of security and the country's enormous development needs are very large in relation to the country's capacity to generate revenue, and its limited room for debt financing. The updated debt sustainability analysis confirms the earlier finding that Afghanistan is at high risk of debt distress and underscores the importance of meeting financing needs with concessional loans and grants, supported by a strong reform agenda. The outlook, however, appears more difficult, as a growing share of the security cost and operating expenditure will be transferred to the domestic budget, and the security cost has been substantially revised upward. As such, fiscal sustainability, as defined in the program, is now projected to be reached in 2032 instead of 2025.

While the authorities are yet to finalize their projections and financing request for the Tokyo conference, they reaffirm their commitment to the reform strategy under the ANDS, which has been endorsed by the donor community, and will seek adequate financing for its full implementation commensurate with the large developmental and poverty-reduction needs and the objectives of maintaining macroeconomic stability and achieving debt sustainability.

\section{Conclusions}

Performance under the ECF-supported program has been adequate and the authorities have demonstrated their commitment to achieving the agreed objectives despite the severe adverse conditions. The authorities are aware that the road toward achieving their development objectives will be long and arduous, and are determined to persevere with reforms in all relevant areas, with adequate donor support. They are highly appreciative of Fund support and technical assistance, but wish to underscore the importance of flexibility and better understanding of the extraordinary difficulties under which reforms are being carried out. 